\title{
Dielectric Lens Antennas
}

Carlos A. Fernandes, Instituto de Telecomunicações, Instituto Superior Técnico, Universidade de Lisboa, Lisboa, Portugal

Eduardo B. Lima, Instituto de Telecomunicações, Instituto Superior Técnico, Universidade de Lisboa, Lisboa, Portugal

Jorge R. Costa, Instituto de Telecomunicações, Instituto Universitário de Lisboa (ISCTEIUL), Lisboa, Portugal

\begin{abstract}
Dielectric lens antennas are attracting a renewed interest for millimetre- and submillimetre wave applications where they become compact, especially for configurations with integrated feeds usually referred as integrated lens antennas. Lenses are very flexible and simple to design and fabricate, being a reliable alternative at these frequencies to reflector antennas. Lens target output can range from a simple collimated beam (increasing the feed directivity) to more complex multi-objective specifications.

This chapter presents a review of different types of dielectric lens antennas and lens design methods. Representative lens antenna design examples are described in detail, with emphasis on homogeneous integrated lenses. A review of the different lens analysis methods is performed, followed by the discussion of relevant lens antenna implementation issues like feeding options, dielectric material characteristics, fabrication methods and a few dedicated measurement techniques. The chapter ends with a detailed presentation of some recent application examples involving dielectric lens antennas.
\end{abstract}

\section{KEYWORDS}

Lens Antennas, Geometric Optics, Physical Optics, Lens Feeds, Dielectric Materials, Lens Manufacturing, Lens Profile Design, Optimization.

\section{INTRODUCTION}

The use of a dielectric lens as part of an antenna is almost as old as the demonstration of electromagnetic waves by Hertz. In fact, in 1888 Oliver Lodge used a dielectric lens in his experiments at $1 \mathrm{~m}$ wavelength (Lodge and Howard 1888). However, it was not until World War II that research on lens antennas has progressed further. Lenses were used to transform the radiation pattern of the primary feed into some high gain radiation pattern, either for fixed or scanning beam applications. But at that time they were supplanted by reflector antennas, less bulky and lighter at microwaves.

With the fast advances on the millimetre and sub-millimetre waves circuit technology for the past two decades, there has been a renewed interest on lens antennas, which present a more acceptable size at these frequencies. Lenses are being explored for 
imaging applications, for fixed and mobile broadband communications, automotive radar applications, among others. In most cases the target radiation pattern continues to be the collimated beam type (plane wave output) either fixed or scanning. But interesting highly shaped beams conforming to demanding amplitude templates have also been explored.

Lenses can be used to modify the phase or the amplitude (or both) of the primary feed radiation pattern in order to transform it into a prescribed output radiation pattern. In this sense lenses are equivalent to reflectors. However, instead of reflection, the lens operation principle is based on the refraction of electromagnetic waves at the lens surfaces (in the case of isotropic homogenous lenses), or within the lens dielectric material in the case of non-uniform refractive index lenses. For instance in one of its most basic configurations, Figure $1(\mathrm{~A})$, parallel rays of an incident plane wave are refracted at the lens surfaces in such a way that all output rays intersect at a point, the lens focal point. All these rays have the same electrical path length, that is, they arrive in phase at the focal point (Fermat's principle), despite their different physical lengths which are compensated by a slower phase velocity $(v=c / n)$ in different portions of the lens. In most designs, lens dimensions are large in terms of wavelength enabling the use of quasi-optical design methods. Large lenses share with reflectors its inherent large bandwidth, which is limited only by the feed bandwidth.

(A)

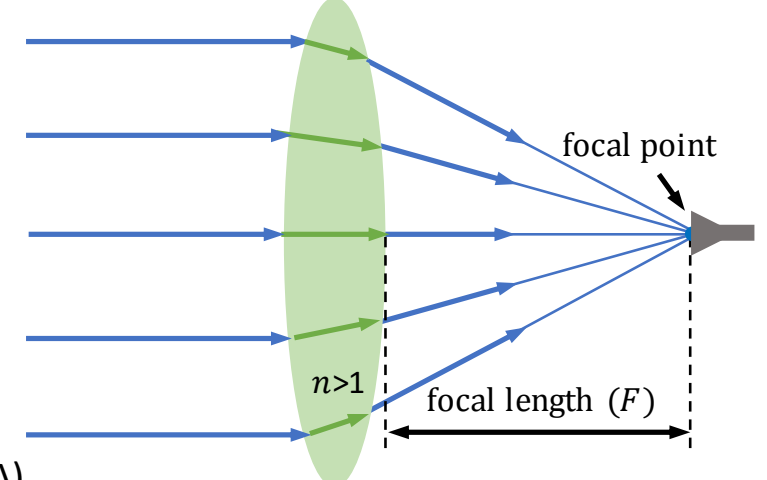

Figure 1 - Focusing antennas: (A) lens; (B) reflector.

One main advantage of lenses over reflectors is that the feed and its supporting structure do not block the antenna aperture. This back feeding feature was key for the development of the millimetre and sub-millimetre wave integrated lens antenna concept where the lens base is positioned directly in contact with the feed, such as an integrated circuit front-end, to produce a directive radiation pattern either single-beam or multiple-beam. The integrated lens structure is very flexible to accommodate demanding output radiation pattern specifications, while multiple shells can be added for instance to increase the design degrees of freedom maintaining a compact structure. This contrasts with multi-reflector systems where blockage issues force large complex structures.

The existence of powerful software simulation tools, numerically controlled machines, 3D additive manufacturing technologies, low-loss dielectric materials and an exploding knowledge on artificial dielectrics are favouring the development and fabrication of very sophisticated high performance lens antennas, namely integrated lens antennas, that 
are easily accessible to most laboratories or companies and affordable for mass market products.

\section{MAIN TEXT}

\section{Lens Theory}

This section starts with a brief overview of different types of known lenses and presents a possible classification. This is followed by some basic concepts applicable for quasioptical lens design, assuming that the lens dimensions and radius of curvature at every point of the surfaces are large compared to the wavelength. Finally this section presents some alternative lens design approaches, involving a combination of iterative algorithms and lens analysis methods.

\subsection{Lens types}

The lens classification adopted in this Chapter is based on three different physical characteristics: the feed position relative to the lens body (far from the lens or in direct contact), refractive index profile (constant/stepped or non-uniform) and number of refraction surfaces, see Table 1 . For each of these categories the lenses can be further classified according to the type of output radiation pattern: fixed-beam (collimated or shaped) or scanning beam (usually collimated).

In early lenses and in few current designs the focal point is located well away from the lens, at a distance comparable with its diameter as in Figure 1(A). These lenses are named in this Chapter the off-body fed lenses and all examples found in the literature are axial symmetric.

Table 1 - Lens classification based on physical characteristics.

\begin{tabular}{|l|l|l|}
\hline \multirow{3}{*}{ 1. Off-body fed } & \multirow{2}{*}{1.1 Homogeneous } & A. Single refraction \\
\cline { 3 - 3 } 2. Integrated & \multirow{2}{*}{2.1 Homogeneous } & C. Multiple refraction \\
\cline { 2 - 3 } & \multirow{2}{*}{2.2 Non-uniform index } & D. Multiple refraction \\
\cline { 3 - 3 } & & E. Continuous refraction \\
\hline
\end{tabular}

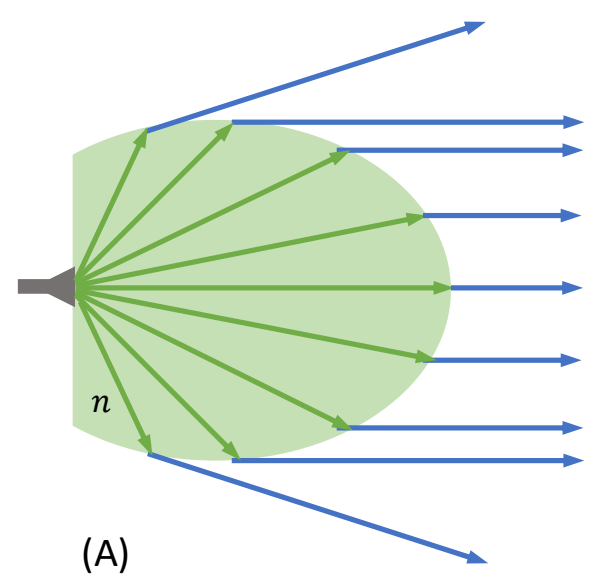

Figure 2 - Examples of integrated lens antennas.

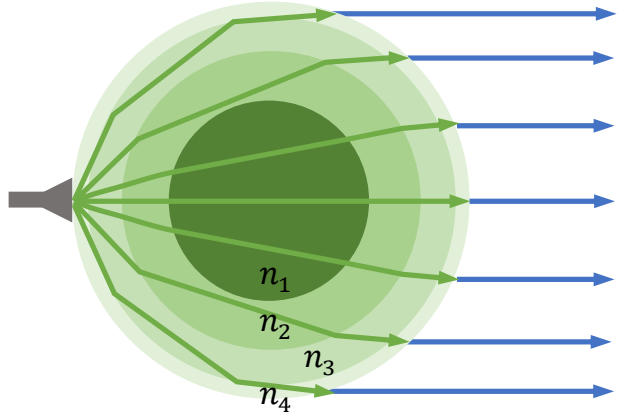

(B) 
Lenses can alternatively be designed to have the feed in direct contact with the lens body (or within the body or at a fraction of the wavelength away). These are referenced in the literature as integrated lens antennas (ILA). These lenses can be made with one or more shells although the most common designs use a single layer.

The concept of integrated lenses started with single material hemispherical lenses added on top of integrated circuit antennas to eliminate substrate modes and increase radiation efficiency (Rebeiz 1992). This has evolved to the use of other fixed canonical shapes like the elliptical or extended-hemispherical to further enhance gain, producing collimated output beams (Filipovic 1993).

But the integrated lens configuration is especially flexible to satisfy more elaborate output beam specifications like secant square type of radiation patterns using more complex lens surfaces (Fernandes 1999), which can have an arbitrary 3D shape in the general case to produce non-symmetrical radiation patterns (Bares 2007). In (Fernandes 2001) the lens shape is adapted to transform the radiation of the circular-symmetrical source into an output shaped beam with square or rectangular footprint when pointed at the ground.

Adding a further shell to the integrated lens adds a second refracting surface offering a supplementary degree of freedom to impose another design condition without sacrificing too much the lens compactness, see Figure 3. For instance in (Costa 2008a) a double-shell lens is designed to satisfy both a beam scanning beam condition and maximum power transfer across the lens surfaces.

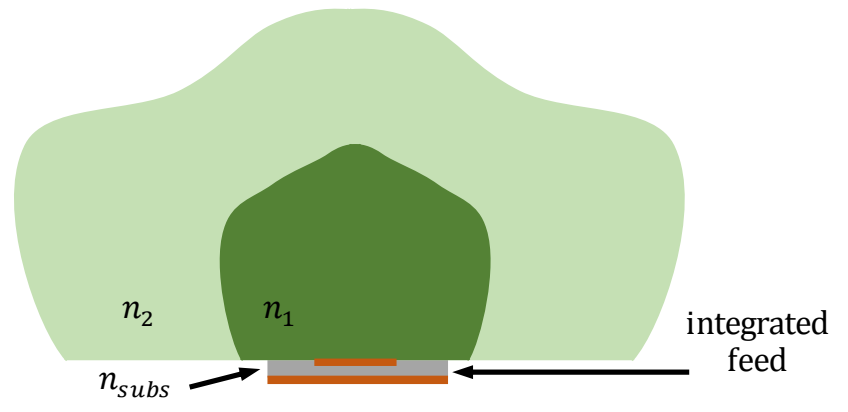

Figure 3 - Shaped integrated dielectric lens antenna.

The Luneburg lens is a special case of integrated lenses, with non-uniform refractive index, where ideally an incident plane wave is focused on the antipodal point on the lens surface, see Figure 2(B).

\subsection{Geometrical Optics for lens design}

Geometrical Optics (GO) is a very convenient formulation for lens (or reflector) design. It derives from the asymptotic solution of Maxwell equations in the high frequency limit (Kay 1965). As long as the overall lens dimensions and surface radius of curvature at any point are much larger than the wavelength, wave propagation inside a homogenous isotropic lens may be conveniently modelled in terms of elementary ray tubes. These emanate from the phase centre of the source, see Figure 4, along straight lines, with the amplitude weighted by the radiation pattern of the source and decaying with path length in the inverse proportion of the square root of the tube cross-section, and with phase given by the electrical path length. Reflection and transmission at an interface are 
made according to Snell's laws (which derive from the Fermat's principle), and ray amplitude is affected by Fresnel coefficients and a divergence factor.

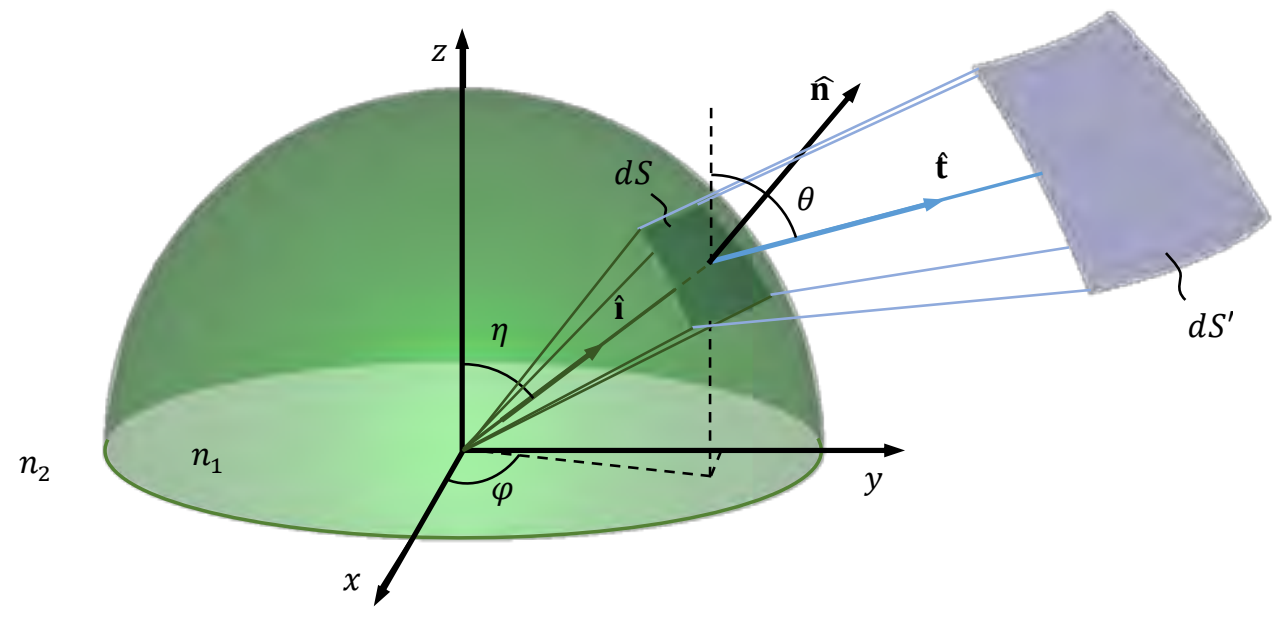

Figure 4-Geometry of the lens and ray tube for GO formulation.

Assuming that the interface between two dielectric media can be considered locally plane, the reflection of an incident plane wave occurs in the same medium, with equal incident and reflected angles - Snell's law for reflection. The refraction is governed by the following Snell's law:

$$
n_{1} \sin \left(\theta_{\text {inc }}\right)=n_{2} \sin \left(\theta_{\text {trans }}\right)
$$

where $n_{1}$ and $n_{2}$ are the refraction indexes of each medium and $\theta_{\text {inc }}$ and $\theta_{\text {trans }}$ are the incidence and transmission angles defined with respect to the interface normal, see Figure 5. If the two media present the same magnetic permeability of air (most of the usual lens materials do), then $n_{1}=\sqrt{\varepsilon_{r 1}}$ and $n_{2}=\sqrt{\varepsilon_{r 2}}$ where $\varepsilon_{r 1}$ and $\varepsilon_{r 2}$ are the relative electric permittivity of each media. The refracted wave is bent towards the surface normal if the wave enters a medium with higher dielectric constant, see Figure $5(A)$, and it is bent away from the normal when exiting a medium with higher dielectric constant, see Figure 5(B).

(A)

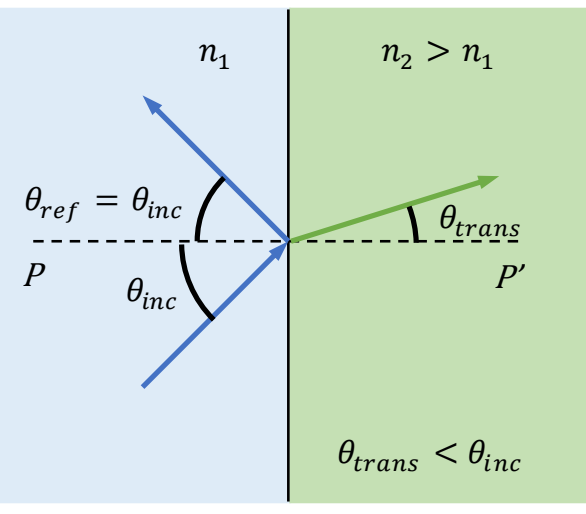

(B)

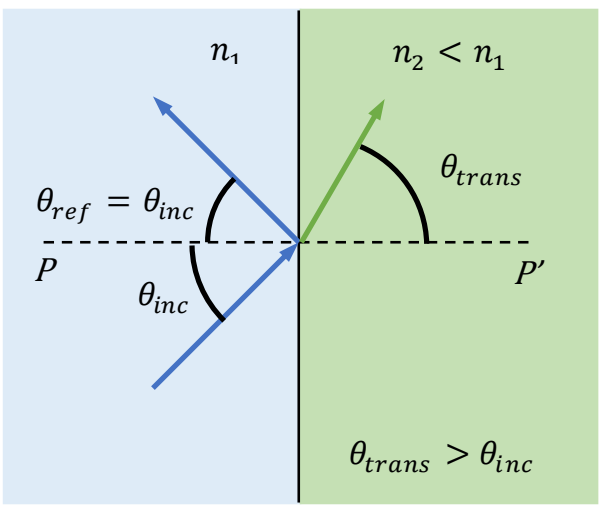

Figure 5 - Plane wave incident at an interface between two dielectrics; $(A)$ incidence from the lower index medium; (B) incidence from the higher index media. 
In the general case the lens surface has an arbitrary curved shape, although with large curvature radius (compared to the wavelength) at any point, and so it is convenient to present the equation (2) in a more general form:

$$
\left(n_{1} \hat{\mathbf{i}}-n_{2} \hat{\mathbf{t}}\right) \times \widehat{\mathbf{n}}=0
$$

where $\hat{\mathbf{i}}$ and $\hat{\mathbf{t}}$ represent the incident and refracted wavenumber directions, respectively (see Figure 4) and $\widehat{\mathbf{n}}$ represents the surface normal. By expressing these three unit vectors in spherical coordinates centred at the feed phase centre, equation (2) for an axial symmetric lens takes the form:

$$
\frac{d r}{d \eta}=\frac{n_{2} r \sin (\theta-\eta)}{n_{1}-n_{2} \cos (\theta-\eta)}
$$

where $r(\eta)$ represents the unknown lens profile while the output angle function $\theta(\eta)$ is the other unknown to be specified by some design condition involving phase, or amplitude or eventually polarization. This condition may be given by an algebraic equation or by a differential equation. The simultaneous equations must be integrated in the interval $\eta \in\left[0, \eta_{\max }\right]$ with applicable initial conditions $r(0)$ and $\theta(0)$ and final value $\eta_{\max }$. This integration is us ually performed numerically, taking only a few seconds to provide the final lens shape.

If other dielectric interfaces are involved in the ray paths, each one must conform to its Snell's equation equivalent to (3). Additional interfaces allow the enforcement of additional radiation pattern specifications which translate into corresponding equations involving the radius $r_{n}(\eta)$ of the $n^{\text {th }}$ interface and the ray exit angle $\theta_{n}(\eta)$. The system of all the involved equations is solved by generalizing the procedure described above for the single interface. It is noted however that the formulation is different for spherical lenses like the Luneburg or Maxwell fish-eye lenses where the unknown function is no longer $r(\eta)$. The refractive index profile $n(r)$ is the unknown function in these lenses. These type of lenses are not the main focus of the present chapter and are treated as specific cases ahead.

The characterization of multiple internal reflections becomes intricate with the increase in the number of lens shells, but in general there is no significant advantage in including its effect in the lens synthesis process. The design formulation must include however the transmission coefficients, divergence factor and material dissipation losses where applicable. Internal reflection analysis can be performed a posteriori when evaluating the lens performance.

Because of lenses large dimensions in terms of wavelength, dissipation losses are also an important aspect to mind. The material permittivity is a complex valued function of the form $\varepsilon_{r}(1-j \tan \delta)$ where the imaginary part inside the parenthesis is the loss tangent. A spherical wave propagating inside the lens material has the form

$$
E_{0}(\theta, \varphi) \frac{e^{-j 2 \pi \sqrt{\varepsilon_{r}(1-j \tan \delta)} \frac{r}{\lambda}}}{r}
$$

So the total dissipation loss depends on lens dimensions, its shape and feed illumination function. For low loss materials, loose bounds for the dissipation loss in a homogeneous lens can be calculated for the minimum and maximum radius $r$ of the lens using 


$$
L=27.3 \sqrt{\varepsilon_{r}} \frac{r}{\lambda} \tan \delta[d B]
$$

Considering that typical lens radius that copes with the Geometrical Optics approximation range from 10 to $30 \lambda$, a value of $\tan \delta=10^{-3}$ corresponds to dissipation losses ranging from about 0.4 to $1.3 \mathrm{~dB}$.

A simple procedure to verify the lens design is to perform a ray tracing, by shooting rays from the feed point at equal angular intervals and using Snell's laws to trace the rays' propagation inside and outside the lens, see Figure 6 . This can reveal for instance the shape of the output phase front, phase centre position or the existence of caustics. The lens radiation pattern in the optical limit can be easily obtained by representing throughout the solid angle the ratio $d S / d S^{\prime}$ of the ray tubes defined in Figure 4, weighted by the feed radiation pattern.

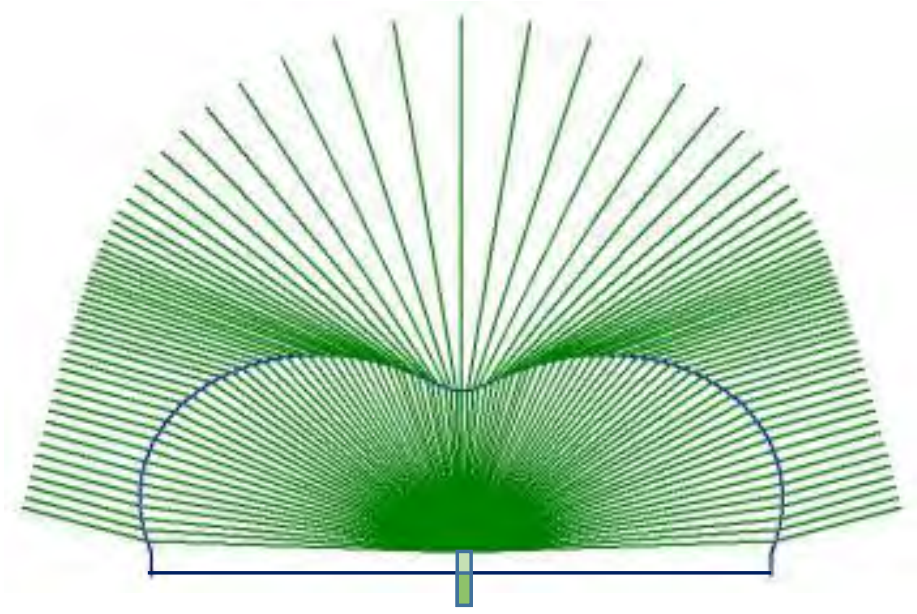

Figure 6 - Ray tracing in a secant squared lens antenna example with embedded feed.

GO based lens synthesis has been used in the literature for different design specifications ranging from simple phase correction problems (Olver 1988) or with additional aperture taper specification, to multi-beam or beam-scanning problems (Kelleher 1961), or to stringent beam-shaping problems (Fernandes 1999). It has been applied for axis-symmetrical lens synthesis as well as for any arbitrary shaped lens (Salema 1998; Fernandes 2001; Sauleau 2006; Bares 2007), for multiple-shell lenses (Silveirinha 2000), for non-uniform refractive index lenses (Cornbleet 1994), or, in fact, for any combination of the previous cases.

The exact solution for the generic 3D structures using GO is numerically involved, but feasible (Salema 1998; Sauleau 2006; Bares 2007). Perturbation methods like the one proposed in (Fernandes 2001) may be simpler to implement for specific types of nonsymmetrical target radiation patterns and it allows to find the appropriate shape for the required non-symmetrical lens. In this reference the lens shape is adapted to transform the radiation of the circular-symmetrical source into an output shaped beam with square or rectangular cross section. An adaptation of the same principle can be used to adjust the lens shape to produce an axis-symmetrical output beam.

\subsection{Other lens design methods}

The advantage of the direct GO synthesis method presented in the previous section is that it provides the lens shape that satisfies the design requirements after elementary 
numerical evaluation of closed form analytical expressions, without the need for any trial and error iterations. Required memory and CPU resources are really modest in the case of axial symmetric lenses. Although the direct GO synthesis is enough for a large number of applications, the method is asymptotic, valid in the optical limit thus neglecting diffraction effects that become important as the lens size reduces.

Alternative lens design methods can be considered when a more accurate lens solution is required for given specifications. They are typically based on a trial and error process involving a parameterized lens model and a lens performance analysis method. The accuracy of the design depends on the accuracy of the numerical modelling and number of required iterations, conditioned by available computational resources. The efficiency of the design process depends very much on how smart the optimization procedure is, due to lens-specific issues which are detailed ahead. Using the direct GO synthesis lens as the first guess for the iterative process may enhance its efficiency.

Before describing the alternative iterative lens design method, it is worth summarizing the following concepts:

- A closed-form lens synthesis method (like the GO method described in the previous section) starts from a list of input parameters and design specifications and directly provides an appropriate lens shape based on a closed form formulation, without the need for trial and error iterations. In this sense this can be further classified as a direct synthesis method. In general the verification of the lens performance requires the subsequent single-time use of a lens analysis method.

- In iterative lens synthesis methods the lens shape is described by some analytical or numerical representation with unknown coefficients which are determined inside an iterative optimization cycle that tests each generated lens using an appropriate lens analysis method until the target specifications are met by trial and error.

- A lens analysis method is intended to evaluate the performance of an existing lens either using an approximate method or a full-wave solver. Its output is the lens performance, not the lens shape.

Unlike direct synthesis methods, the list of available analysis methods is considerably larger. The most used are presented in Table 2, grouped according to the type of electromagnetic modelling. A brief description of these methods is provided in Section 2.

Table 2 - Possible lens antenna analysis methods.

\begin{tabular}{|c|c|}
\hline \multirow{4}{*}{ Approximate } & Geometrical Optics / Physical Optics (GO/PO) \\
\cline { 2 - 2 } & Physical Optics / Physical Optics (PO/PO) \\
\cline { 2 - 2 } & Spectral Domain Method (SDM) \\
\hline \multirow{4}{*}{ Full Wave } & Spherical Wave Modal Method \\
\cline { 2 - 2 } & Finite Element Method (FEM) \\
\cline { 2 - 2 } & Method of Moments (MoM) \\
\cline { 2 - 2 } & Finite-Difference Time-Domain (FDTD) \\
\hline
\end{tabular}


Figure 5 presents a schematic diagram of the two mentioned approaches for lens design - the direct synthesis method on the left and the iterative synthesis methods on the right. Naturally both start from the definition of the target specifications, lens material and primary feed characteristics. The path on the left provides a fast first guess of the solution, which can be enough for certain problems or can be fed for refinement throug $h$ the iterative process on the right.

Two alternative approaches are considered for the parametric modelling of the lens. A polynomial type representation can be adopted, where the coefficients are the unknowns to be optimized in the loop instead of the large collection of the lens surface coordinates. This solution is very flexible allowing the representation of arbitrary shapes. The disadvantage is that the optimization algorithm may generate an unnecessary number of useless lenses, not because the geometry is impossible (it can be checked before starting the lens analysis and removed from the test population) but because randomly generated lenses can easily originate total internal reflection, surface wave modes and caustics, especially for integrated multiple-shell lenses which can only be detected after the analysis process.

In the second approach (Lima 2008), the search space can be narrowed to specific classes of lenses by using the analytical lens profiles from the GO synthesis method, but letting the involved parameters be managed by the optimization algorithm. The analytical-based solutions ensure the electromagnetic viability of all the solutions tested in the iterative process. There is a convergence time versus design flexibility trade-off between these two approaches.

The described procedures and the workflow presented in Figure 7 are implemented in a freeware lens design, analysis and optimization tool - the ILASH software tool (Lima 2008). It was developed for circular symmetric shaped integrated lens antennas with single or double layer, and can handle multiple target specification definitions. The lens analysis method is based on the GO/PO and the optimization is based on genetic algorithms. A screenshot of the ILASH user interface is presented in Figure 8. It allows simple interaction with the kernel, to generate and manipulate lens design data, to fully characterize the lens performance and to export and import results. It is also possible to monitor in real time several aspects of the optimization process like the cost function value, lens parameter evolution and convergence. ILASH was used to design the lens examples described in Section 3. 


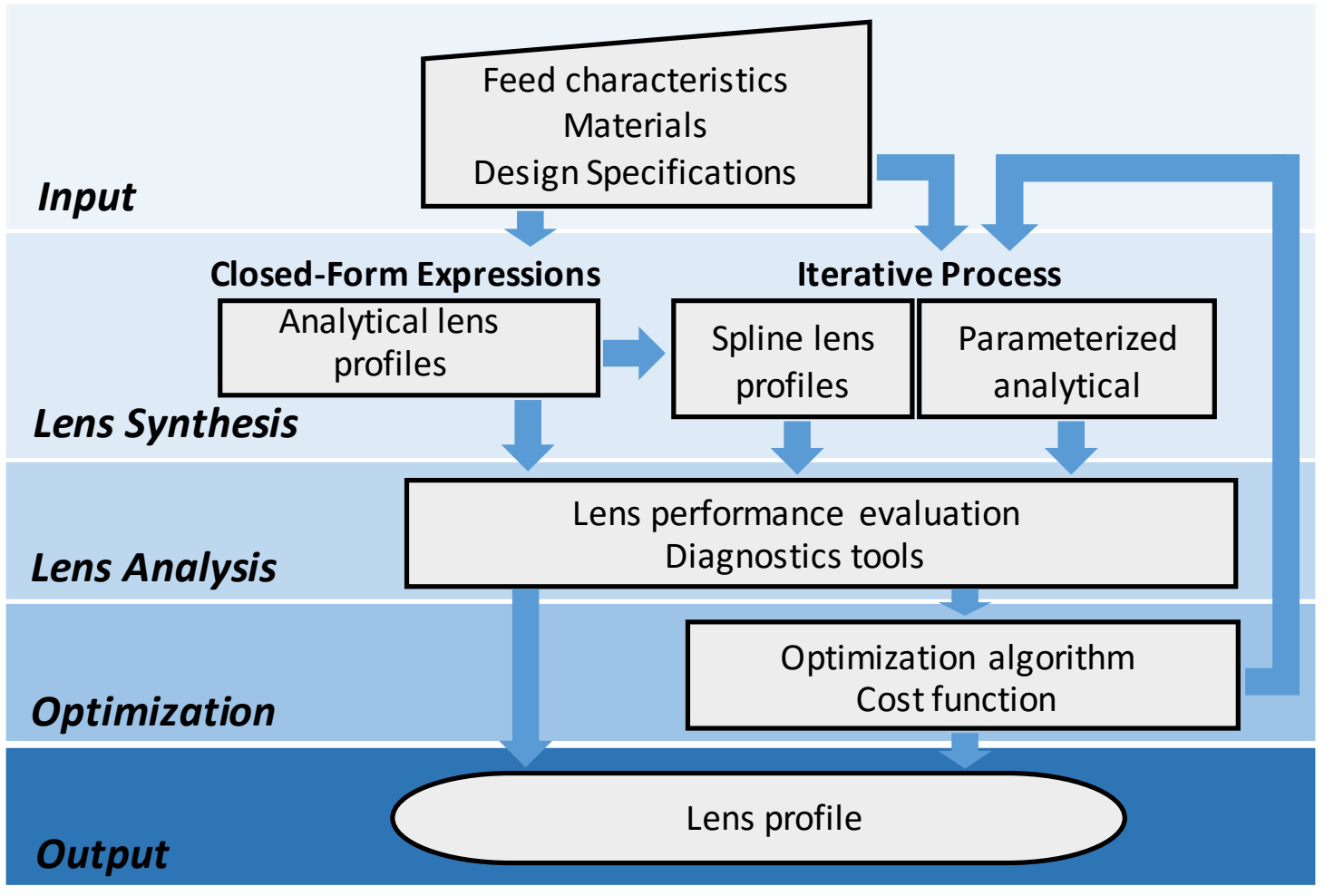

Figure 7 - Block diagram of lens design steps.

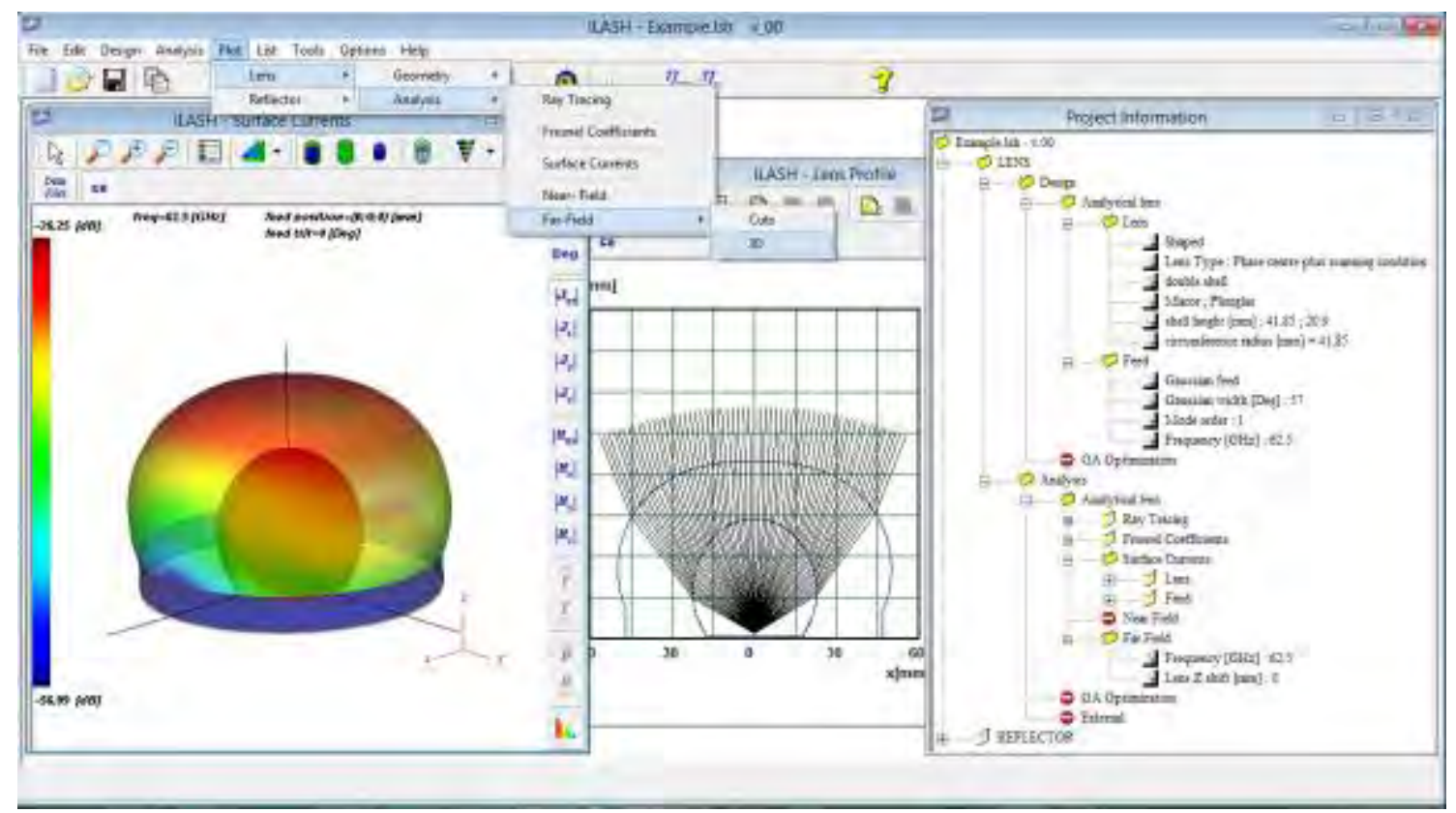

Figure 8 -Screenshot of the user interface of the freeware ILASH software.

\section{Lens Design, Fabrication and Test}

This section presents a few representative examples of lens design, organized according to the lens classification introduced in Section 1. In most of the cases the design is based on $\mathrm{GO}$ formulation. The section includes also a discussion of the steps that follow the lens design towards fabrication and test. 


\subsection{Off-body fed lens design examples}

Early works on dielectric lens antennas were based upon the concepts of optical lenses. Most of the late 1800 and early 1900 tests were conducted with off-body fed lenses which were used to collimate the radiation of a plane wave into the feed placed at the focal point in the opposite side of the lens. Also most off-body fed lenses have an axial symmetric shape and, at least during design, the feed radiation pattern is considered also circularly symmetric. These assumptions remove the dependence of the lens with the $\phi$ angle, the angle of rotation about the antenna axis of symmetry. Off-body fed lens antennas are usually made of a single material and can have either one or two refracting surfaces.

\subsubsection{Single refraction lenses}

The simpler lens configurations with a single refracting surface are the elliptical and the hyperbolic lenses, see Figure 9. In an elliptical lens the surface closer to the feed (inner surface) has a spherical shape and does not refract the rays. The collimation of the rays is achieved from the elliptical surface farther away from the feed (outer surface). In a hyperbolic lens the refraction occurs in the hyperbolic lens surface closer to the feed. In this configuration the outer lens surface is planar and does not refract the rays.

(A)

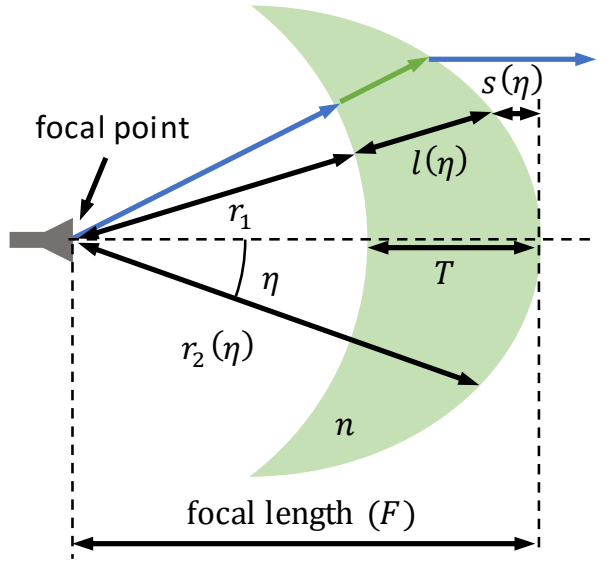

(B)

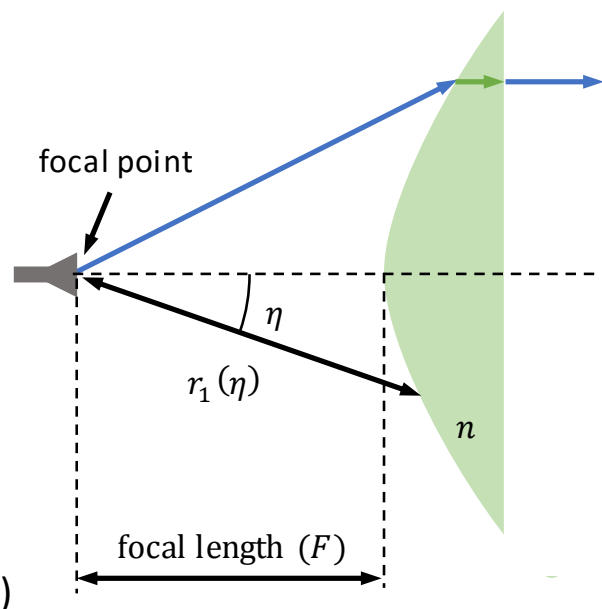

Figure 9 - Off-body fed lens examples. (A) Elliptical lens; (B) Hyperbolic lens.

The shape of the outer surface of the elliptic lens in polar coordinates can be obtained by imposing the path length collimation condition:

$$
r_{1}+n l(\eta)+s(\eta)=r_{1}+n T
$$

and the following physical length condition:

$$
\left[r_{1}+l(\eta)\right] \cos (\eta)+s(\eta)=r_{1}+T
$$

where $T$ is the lens selected thickness at the axis. Subtracting the two equations and noting that $r_{1}+l(\eta)=r_{2}(\eta)$ and that $r_{1}+T=F$ where $F$ represents the lens focal distance, the outer surface profile is expressed as:

$$
r_{2}(\eta)=\frac{F(n-1)}{n-\cos \eta}
$$


where $n$ is the refraction index of the lens material and $F$ is the lens focal distance. Snell's equations are implicitly satisfied by the collimation condition (which translates Fermat's principle).

The inner shape of the hyperbolic lens can be obtained using a similar set of equations and is defined by

$$
r_{1}(\eta)=\frac{F(n-1)}{\cos \eta-1}
$$

Note that the design is based solely on phase considerations, assuming a point source. In the ideal case when both lenses types with identical diameters are fed with a point source, the elliptical lens presents higher directivity and lower side lobe levels. In fact, all the inner surface points of the elliptical lens are at the same distance from the source and are illuminated with identical amplitude. The main disadvantage of the elliptical lens is that the reflection at the inner surface of the lens, due to the contrast between the air and the dielectric permittivity, is normal to the spherical surface and is reflected back towards the feed (Piksa 2011). In the hyperbolic lens, the diffraction effects at the edges of the lens tend to influence more the main beam of the radiation pattern and increase the side lobe level (Piksa 2011). The hyperbolic lens however may be simpler to manufacture since it presents one planar surface.

\subsubsection{Double refraction lenses}

Lenses with two refracting surfaces allow more control of the radiation pattern characteristics as discussed in Section 1.2. In fact, based upon geometric optics a set of differential and linear equations can be obtained to determine the coordinates of one of the surfaces (Silver 1984). However, those equations are not sufficient to determine uniquely both surfaces so another design condition must be imposed.

One interesting use for the extra degree of freedom provided by the second refracting surface is in scanning beam applications. If the feed in the previous elliptical or hyperbolic lenses is shifted off the focus on a plane normal to the lens axis, the beam tilts linearly for very small shifts and with a more complex dependence for larger shifts along with increasing beam deformations. In fact the phase front of the output beam is no longer plane for off-axis feed positions. Expanding it in Taylor series reveals a linear term responsible for the linear tilt and non-linear terms responsible for different types of superimposed beam deformations (Born 1959).

It is possible to extend the linear tilt angle range for scanning beam applications by imposing the so called Abbe sine condition for the design of the second available lens refracting surface. This enables designing a collimating lens which is free from comma aberration when the feed is transversely displaced away from the lens axis (Born 1959). The inner surface of the lens can be defined by the unknown function $r_{1}(\eta)$ and the outer surface by the unknowns length $I(\eta)$ and angle $\gamma(\eta)$ represented in Figure 10 . The lens focal length is $F$ and the axial thickness is $T$. The Abbe sine condition is verified when the intersections of the extended $r_{1}(\eta)$ rays departing from the lens focal point and the corresponding extended transmitted $s(\eta)$ rays all lie over an arc of circumference with a certain radius $f_{\mathrm{c}}$ centred at the focal point. This is represented by the thick dashed line in Figure 10. In view of the geometry presented in Figure 10, the Abbe sine condition can be written as 


$$
\left(f_{e}-r_{1}\right) \sin (\eta)=l \sin (\gamma)
$$

The Snell's law at the inner surface of the lens implies that (equation (3))

$$
\frac{d r_{1}}{d \eta}=\frac{n r_{1} \sin (\gamma-\eta)}{1-n \cos (\gamma-\eta)}
$$

In order that the electrical path length of every ray is the same at the exiting wavefront, it is required that

$$
\begin{gathered}
r_{1}+n l+s=F+n T, \\
s+r_{1} \cos (\eta)+l \cos (\gamma)=F+T
\end{gathered}
$$

Equations (10)-(13) can be solved simultaneously for $r_{1}(\eta), l(\eta), s(\eta)$ and $\gamma(\eta)$ taking $\eta$ as the independent variable and the initial conditions $r_{1}(0)=F, l(0)=T, s(0)=0$ and $\gamma(\eta)=0$. The outer surface of the lens can be calculated from the previous four functions.

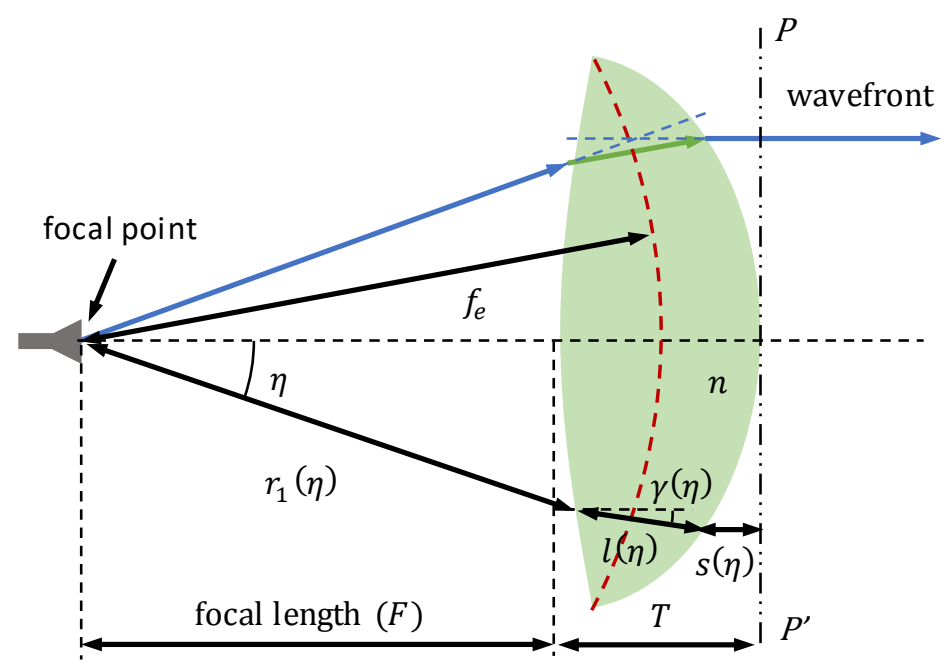

Figure 10-Geometry of a two refracting surface lens for Abbe sine condition enforcing.

\subsubsection{Zoning}

Usually lenses are several wavelengths thick, particularly near the lens axis. For microwave applications this may lead to a very bulky antenna and originate nonnegligible dissipation losses in the dielectric. To attenuate those effects, rings of material with a thickness equal to an integer multiple of the wavelength can be removed from the lens, a process called zoning. Figure 11 shows an example for the hyperbolic lens discussed in section 2.1.1 with four zones $(K=4)$. A minimum physical lens thickness $t_{m}$ needs to be maintained in the zoned lens to provide structural support, as indicated in Figure 11. The thickness of the zoned lens is independent of the number of zones $K$ and it is given by $t_{m}+\lambda_{0} /(n-1)$, which for the example in Figure 11 represents about a quarter of the original lens thickness. 

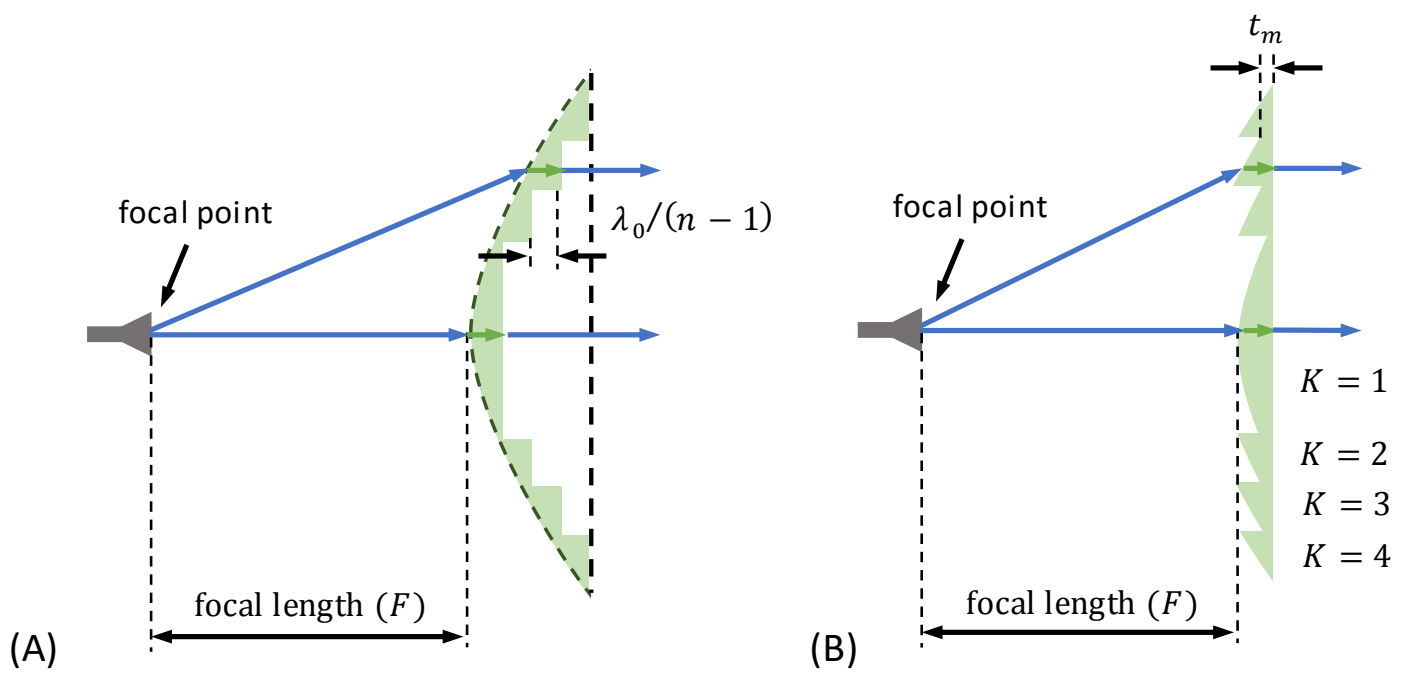

Figure 11 - Zoning of a hyperbolic lens (A) Original outer hyperbolic shape; (B) Corresponding zoned lens.

The downside of zoned lens is the associated frequency dependence which becomes more important as the lens diameter increases and more zones are used. A criteria to estimate the bandwidth of zoned lenses is given by (Silver 1984): under uniform aperture distribution and lens aperture phase error smaller than $\pi / 4$

$$
\text { Bandwidth } \approx \frac{25 \%}{K-1}
$$

If the zoning is performed in the non-refracting surface of the lens, shadowing losses can appear at the transition regions and this effect is stronger for lenses with larger ratios between the focal length and the lens diameter F/D (Petosa 2000).

\subsection{Integrated lens design examples}

This section addresses briefly two classical examples of non-uniform refractive index lenses - the Luneburg lens and the Maxwell fish-eye - and focuses more on the homogeneous shaped lenses. Other non-uniform refractive index lenses have gained also interest with the recent advances in the fields of transformation optics and metamaterials but these exceed the scope of this chapter.

Homogeneous integrated lenses are very effective for integration with low cost millimetre wave circuits, allowing low form-factor solutions with sophisticated radiation pattern characteristics owing to the great design flexibility this lens configuration provides. However, internal reflections may critically influence the lens performance and must be properly analysed in each design. This section presents some representative design examples of canonical lenses as well as some more recent shaped lenses based on GO.

\subsubsection{Non-uniform index spherical lenses}

In the classical non-uniform spherical lenses the dielectric constant of the material has only radial variation. Therefore, the lens is symmetric in relation to any axis passing the centre of the lens. As a consequence, these spherical lenses do not present a unique focal point but rather a spherical surface concentric with the lens where the focal point can be located depending on the direction of the incident wave. 
The most known of the spherical lenses is the Luneburg lens (Luneburg 1943) where the lens material permittivity profile varies with the square of the $r$ distance from the lens centre

$$
\varepsilon_{r}(r)=2-(r / R)^{2}
$$

being $R$ the outer radius of the lens. This formulation results in a focal region located at the outer surface $r=R$ of the lens. Therefore, a point source located at any point of the lens surface originates a collimated beam in the opposite direction, Figure 12. This property is independent of the lens diameter. The Luneburg lens is particularly adequate for multi-beam applications since the symmetry ensures that all beams are equal independent of the feed position on the lens surface.

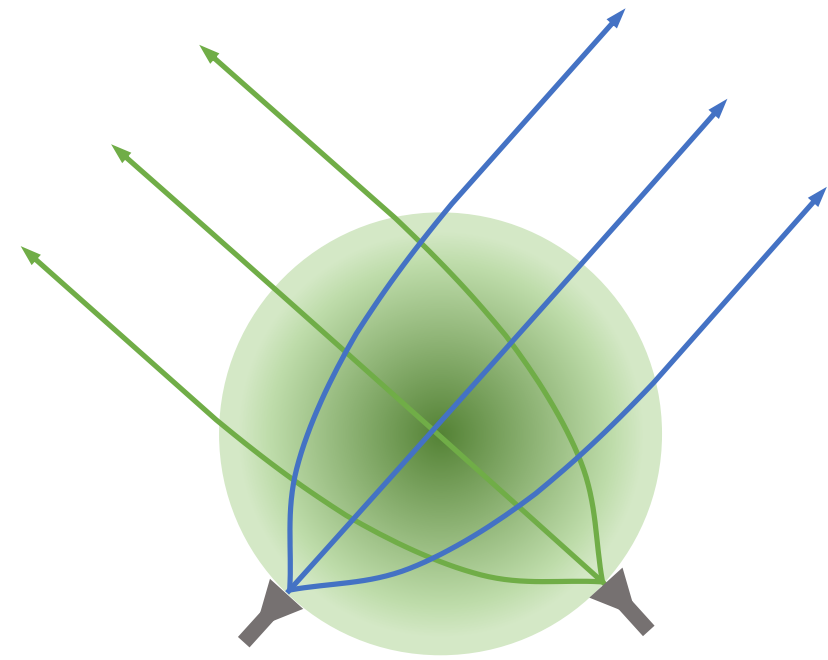

Figure 12 - Luneburg lens with darker colour representing increasing permittivity.

Luneburg did not have the opportunity to demonstrate such antenna experimentally since no suitable lens materials were available at the time. Nowadays, Luneburg lenses are formed by discrete number of concentric dielectric layers that approximate the ideal permittivity law of equation (15). It is expected that as the number of discrete layers increases the performance of the lens improves; however, the manufacturing complexity increases as well as the influence of possible air gaps between the layers (Kim 1998). It has been demonstrated that a quite small number of shells is enough to obtain a directivity and side lobe level close to the ideal case (Fuchs 2007). Nevertheless, the recommended number of discrete layers increases with the lens diameter compared to the wavelength. For example, just 6 layers were required to achieve adequate performance with an $8 \lambda_{0}$ diameter Luneburg lens (Fuchs 2007a). Even lesser number of layers is possible by optimizing both the permittivity and thickness of the layers (Mosallaei 2001; Boriskin 2011).

There are also examples of Luneburg lenses fabricated from a single material with controllable dielectric constant. For instance in (Min 2014) a 3D rapid prototyping machine was used to achieve the desired permittivity value by controlling the filling ratio of a polymer/air-based unit cell. Alternatively, it is possible to use foam material and press it to obtain the desired dielectric constant. In fact, when the pressure increases and the foam is compacted, the quantity of air in the material decreases increasing the permittivity. This technique has been tested for the fabrication of a Luneburg lens in (Bor 2014). It is also possible to control the dielectric constant in the Luneburg lens by 
drilling holes into the material and varying either the diameter of the holes or their density (Sato 2002; Xue 2007).

One of the major disadvantages of the Luneburg lens is its high profile. Transformation optics (Do-Hoon 2010) can be used to change the shape of the lens into a much lower cylindrical profile. However, common solutions end up resulting in materials that are anisotropic and/or must have relative permeability different than unity forcing the use of metamaterials that are usually narrowband, lossy, dispersive and sometimes cumbersome to manufacture. A reasonable performance has been achieved with a compacted Luneburg lens with cylindrical geometry, formed by discrete layers with step permittivity variation along the radius and along the height (Mateo-Segura 2014). Although a height reduction was obtained, permittivity values as high as 12 are required, and the structure presents some scanning loss.

A commonly used solution to reduce the profile of the Luneburg lens by a factor of 2 , is to combine half of the lens with a flat ground plane, Figure 13. The ground plane creates an image of the upper hemisphere and simulates the complete Luneburg lens. The size of the ground plane must be adequate to produce the required image, and the necessary dimensions are function of the elevation angle of the beam in relation to the ground plane. An insufficient ground plane size will reduce the directivity of the beam and cause scanning loss. This hemispheric lens solution also suffers from feed blockage for high elevation angles. One advantage of this configuration is that it is easier to mechanically stabilize than the complete spherical lens. Recently a quarter Luneburg lens with a $90^{\circ}$ degree corner ground plane has been demonstrated (Nikolic 2012).

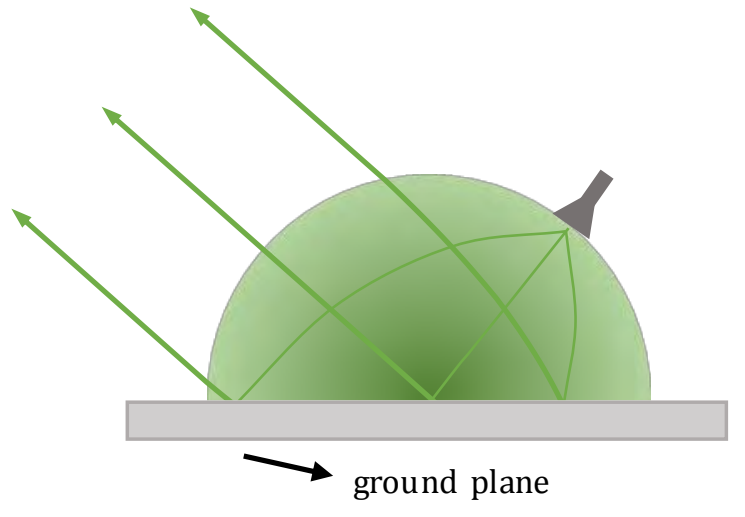

Figure 13 - Half hemisphere Luneburg lens.

A similar configuration to the Luneburg spherical lens is the cylindrical one, Figure 14, where the variation of the dielectric constant occurs only in the radial direction. In this solution the beam collimation is obtained only in one plane, leading to fan shaped beams instead of pencil like beams (Komljenovic 2010). 


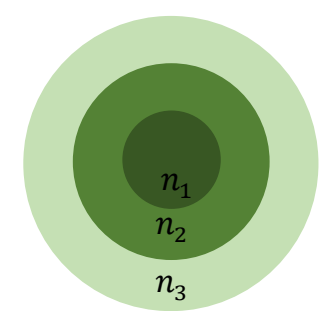

(A)
(B)

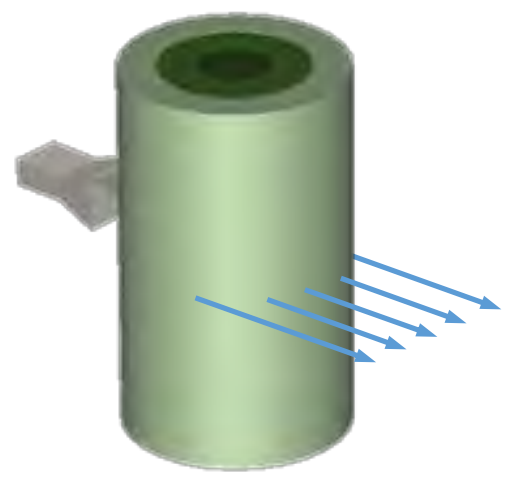

Figure 14 - Cylindrical Luneburg lens.

Another classical non-uniform index spherical lens is the Maxwell's fish-eye lens, which pre-dates the Luneburg lens (Maxwell 1860). The dielectric constant of the lens material is given by

$$
\varepsilon_{r}(r)=\left(\frac{4}{\left[1+(r / R)^{2}\right]^{2}}\right)
$$

where $R$ is the outer radius of the lens. When a point source is placed on the surface of the lens, the radiation is focused at the antipodal point of the lens as represented in Figure $15(\mathrm{~A})$. Due to the lens symmetry, the source radiation is converted into a local plane wave at the centre of the lens. Therefore, if the Maxwell's fish-eye lens is cut in half it can be used as a collimating lens that focuses a plane wave into a focal point on the surface of the lens, which becomes unique in this case, Figure 15(B). The halved Maxwell's fish-eye lens (HMFE) has lower profile than the Luneburg lens and is easier to flush mount. Practical implementation of the HMFE has been done using a few discrete layers (Fuchs 2006) and the performance was shown to be comparable with the Luneburg lens when the feed is centred with the lens (Fuchs 2008).

(A)

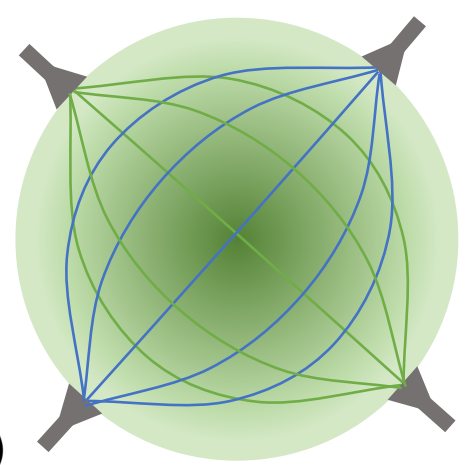

(B)

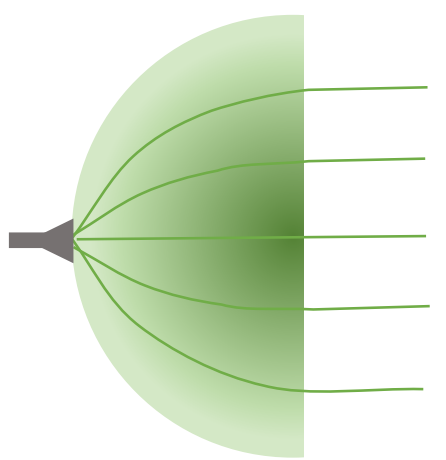

Figure 15 - (A) Maxwell fish-eye lens; (B) Half Maxwell fish-eye lens. The darker colour represents higher values of permittivity.

Unlike the Luneburg lens, the HMFE lens loses interest for scanning beam applications since scanning losses appear as the feed is moved over the lens surface (Fuchs 2007b).

\subsubsection{Elliptical and hemispherical lenses}

The homogeneous elliptical integrated lens, justlike its off-body fed counterpart treated in Section 2.1.1, is used to transform the radiation pattern of a feed placed at the focal point of the lens into a plane wave in the air medium, propagating along the lens axis, 
Figure 16. It can be viewed as the limit when $r_{1}$ tends to zero in equations (6)-(7) from Section 2.1.1:

$$
n r(\eta)+l(\eta)=n F
$$

Also the following physical length condition can be imposed:

$$
r(\eta) \cos \eta+l(\eta)=F
$$

Eliminating $l(\eta)$ between the two equations leads to the same elliptical lens profile found in Section 2.1.1:

$$
r(\eta)=\frac{F(n-1)}{n-\cos \eta}
$$

It is a simple matter to show that this corresponds in rectangular coordinates to

$$
\left(\frac{x}{a}\right)^{2}+\left(\frac{z-L}{b}\right)^{2}=1
$$

where $a$ is the radius along the $x$-axis, $b$ is the radius along the z-axis, $L$ is the position of the focal point in relation to the centre of the lens and $b+L=F$, Figure $16(\mathrm{~A})$. The eccentricity of the elliptical lens is related to the dielectric constant of the material. The following relations hold (Filipovic 1993)

$$
\begin{gathered}
b=\frac{a}{\sqrt{1-1 / n^{2}}} \\
L=b / n
\end{gathered}
$$

The value of $a$ is chosen to define the lens size, and consequently the aperture size required to achieve a certain directivity of the beam.

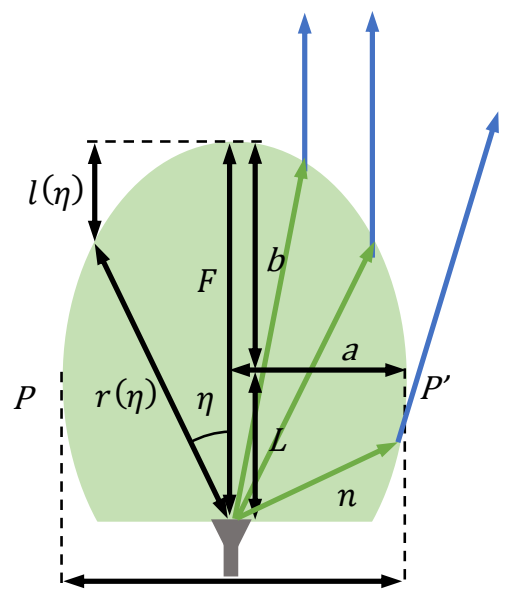

maximum-waist
(A)

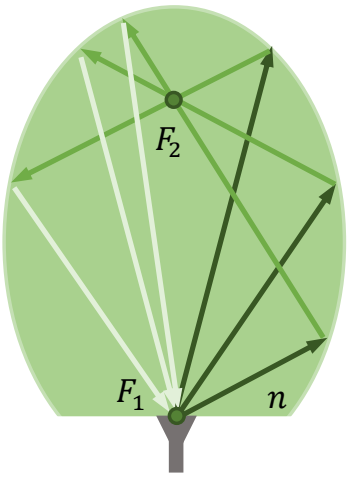

(B)

Figure 16 - (A) Design variables of an elliptical integrated lens antenna; (B) rays reflection inside the lens.

Only the rays that hit the lens surface above the plane of maximum waist $P P^{\prime}$ are focused, Figure 16(B). The feed radiation intersecting the lens below the maximum waist are not collimated but rather propagate along undesired directions or excite surface wave modes (Pasqualini 2004) giving rise to side lobes or other perturbations in the lens 
radiation pattern. For this reason, proper feed configurations should be used to minimize the illumination of the lower part of the lens surface.

Internal reflections may be especially critical in integrated lens antennas. For instance if the feed is at the centre of an hemispherical lens, all reflected rays travel back along the same path of the corresponding incident rays, concentrating at the focal point, usually causing a substantial mismatch at the feed impedance. A similar effect occurs in the elliptical lens; all reflected rays concentrate at the feed point after travelling through the second focal point of the elliptical lens, originating a second order reflection at another point of the lens surface, Figure 16(B). (Neto 1998; Neto 1999; Van Der Vorst 1999; Van Der Vorst 2001). Again, this causes feed impedance mismatch. Part of the second order ray is transmitted to the air at an undesired direction causing side lobes in the lens radiation pattern. These undesired effects increase with the contrast between the material and the air refraction indexes. For other integrated lens shapes the reflected rays may not be all reflected back into the feed, nevertheless, they may be responsible for transmission of higher than first order rays along undesired directions, reducing the main beam efficiency or causing ripple.

The problem of internal reflections can be tackled, at least within a limited bandwidth. For normal incidence on a planar interface between two dielectric media with refraction index $n_{1}$ and $n_{2}$, it is possible to cancel the reflected ray by adding an appropriate intermediate layer, Figure $17(\mathrm{~A})$. Its dielectric refraction index $n^{\text {match }}$ must be

$$
n^{\text {match }}=\sqrt{n_{1} n_{2}}
$$

and a thickness $h$

$$
h=\frac{\lambda_{0}}{4 n^{\text {match }}}
$$

where $\lambda_{0}$ is the free space wavelength. This intermediate layer is usually called the matching layer.

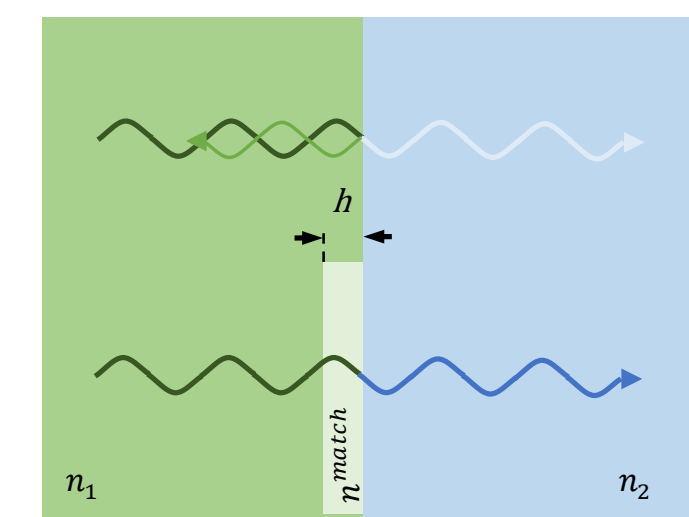

(A)

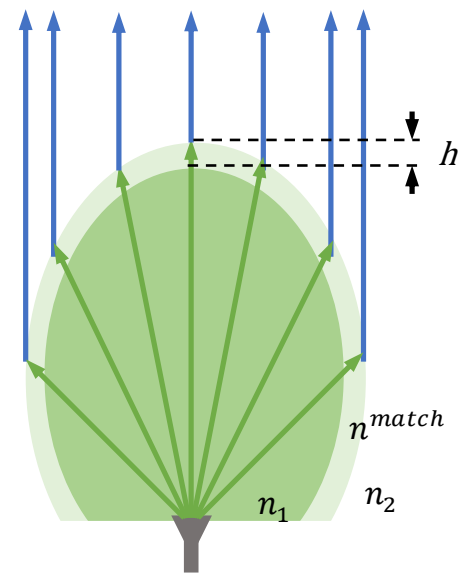

(B)

Figure 17 - Matching layer (A) in a planar dielectric air interface. The intensity of ray color is proportional to the wave power density; $(B)$ in an elliptical integrated lens antenna

By adding a matching layer to the lens surface it is possible to alleviate the effect of internal reflections, Figure $17(B)$. It is a fact that the rays do not hit the lens surface along 
the normal direction and therefore the thickness of the matching layer should not be given by (24). Instead it should vary along the surface of the lens according to the ray incident angle. In a study presented in (Van Der Vorst 1999) for an elliptical integrated lens of silicon $\left(\varepsilon_{r}=11.7\right)$ it was demonstrated that there is no major improvement on the lens radiation performance by using the optimum thickness matching layer. Therefore, a constant thickness layer given by (24) is generally used, facilitating the manufacturing process. Because it is not easy to find natural materials with specific permittivity values, it is common to synthesize an effective medium layer by periodically removing a fraction of the dielectric material from the lens surface, such as drilling holes or cutting groves (Ngoc Tinh 2010).

The matching layer thickness is frequency dependent and, therefore, reduces the bandwidth of the lens antenna. To improve the bandwidth multiple consecutive matching layers can be used performing a gradual transition between the two dielectric constants at the interface (Ngoc Tinh 2009).

The extended hemispherical lens is another classical configuration of integrated lens, owing to its simple shape for fabrication. It consists of a half sphere of radius $R$ with a cylindrical extension of height $L$. The feed is located at the base of the lens, Figure 18(B).

(A)

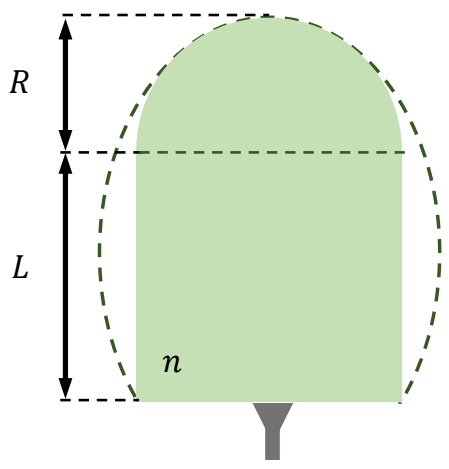

(B)

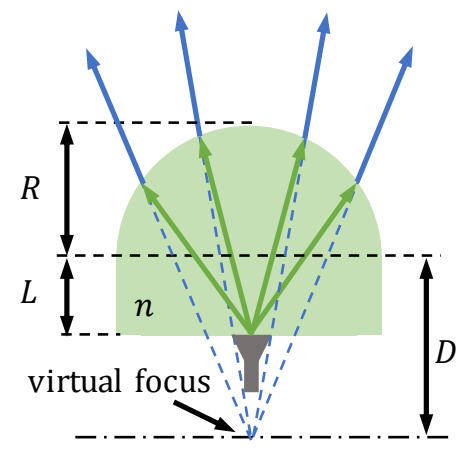

Figure 18 - Extended hemispherical integrated lens antenna: (A) synthesized elliptical; (B) hyper hemispherical.

If the length of the cylindrical extension is chosen to be

$$
L=\frac{R}{n-1}
$$

most of the spherical part of the hyper hemispherical lens coincides with an elliptical lens, Figure 18(A). This lens is usually called synthesized elliptical lens (Filipovic 1993) although it tends to present a slightly lower directivity when compared to a true elliptical lens with the same diameter.

A second type of extended hemispherical lens is the hyper hemispherical (Rebeiz 1992) where the cylindrical extension length is

$$
L=R / n
$$

For this particular type of lens the output beam is not collimated, presenting a much broader (and sometimes multi-lobed) radiation pattern when compared to an elliptical lens with the same radius. Nevertheless, the hyper hemispherical lens bends the rays radiated by the feed toward the axis of the lens, Figure $18(\mathrm{~B})$. The lens sharpens the 
radiation pattern, effectively increasing the gain of the feed by a factor $n^{2}$ (Rebeiz 1992). However, unlike collimated lenses, the directivity of this lens does not increa se with lens size (or aperture size).

The hyper hemispherical lens satisfies the Abbe sine condition (Born 1959). Therefore, this lens is free from comma aberration when the feed is transversely displaced within certain limits away from the lens axis. This type of lens is particularly useful for beam scanning applications. Another interesting characteristic of hyper hemispherical lenses is that all the rays transmitted into the air focus in a virtual point behind the lens at a distance

$$
D=R n
$$

from the centre of the spherical part of the lens. This means that the radiation pattern of this lens presents a very stable phase centre position coincident with this virtual focus and its position does not shift with the frequency (in the optical limit).

There are several other types of shaped dielectric integrated lens antennas with profiles not given by canonical expressions like the elliptical and extended hemispherical lenses. In section 3 of this chapter several design and implementation examples of noncanonical shaped lenses will be given in more detailed.

\subsubsection{Lens designed to match an output power template}

Integrated lenses can also be used to conform the output radiation pattern to some farfield power template $G(\theta)$. The formalism of a GO-direct synthesis method is described for an axial-symmetric single material lens with refraction index $n$, fed on-axis at the base of the lens as shown in Figure 19 (Fernandes 1999).

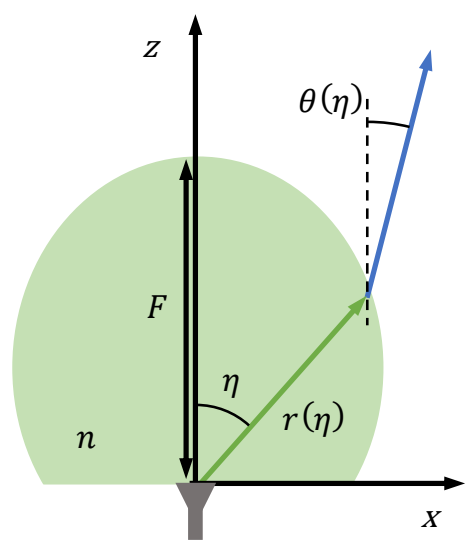

Figure $19-$ Geometry of a single-shell lens.

The lens design requires the knowledge of the feed power pattern $U(\eta)$ when the feed is inside the lens material. This can be obtained using a full-wave analysis of the feed radiating into an unbounded medium with the same permittivity as the lens. Alternatively it can be obtained experimentally using the procedure described in Section 2.7 .

The unknown lens profile is represented by the $r(\eta)$ function. As before, the Snell's equation for refraction at the dielectric interface is written as: 


$$
\frac{\partial r}{\partial \eta}=\frac{r(\eta) \sin (\theta-\eta)}{n-\cos (\theta-\eta)}
$$

In order to enforce the output power pattern template, the elementary ray tube concept introduced in Section 1.2 is used, Figure 4. Power conservation in the elementary ray tube is expressed as

$$
U(\eta) T \sin \eta d \eta=K G(\theta) \sin \theta d \theta
$$

or rearranging

$$
\frac{d \theta}{d \eta}=\frac{T}{K} \frac{U(\eta)}{G(\theta)} \frac{\sin \eta}{\sin \theta}
$$

where $T(\eta)$ represents the transmissivity, that is, the ratio of the power $P_{t}$ transmitted across $d S$ to the incident power $P_{i}$ (Figure 4).

$$
\begin{gathered}
T=\frac{U_{\|}\left|t_{\|}^{2}\right|+U_{\perp}\left|t_{\perp}^{2}\right|}{U} \frac{1}{n} \frac{\cos \beta}{\cos \alpha} T \\
\cos \alpha=\hat{\mathbf{i}} \cdot \widehat{\mathbf{n}} \\
\cos \beta=\hat{\mathbf{t}} \cdot \widehat{\mathbf{n}}
\end{gathered}
$$

where $t_{/ /}$and $t_{\perp}$ represent the Fresnel transmission coefficients for parallel and perpendicular polarization respectively. $K$ is a normalization constant to be determined from the balance between the total power inside the lens and total power outside the lens

$$
K=\frac{\int_{0}^{\eta_{\max }} T(\eta) U(\eta) \sin \eta d \eta}{\int_{0}^{\theta_{\max }} G(\theta) \sin \theta d \theta}
$$

being $\eta_{\max }$ the maximum feed aperture and $\theta_{\max }$ the maximum output angle.

The unknowns $r(\eta)$ and $\theta(\eta)$ are obtained by integrating the system of equations formed by (28) and (30) from $\eta=0$ to $\eta_{\max }$ (typically $90^{\circ}$ ) using the initial condition $r(0)=F$ and $\theta(0)=0 . F$ acts as a scaling factor, not affecting the lens shape. However, the larger is this value the larger is the lens size. Inherent to the design, increasing the size also improves the lens radiation pattern compliance with the target $G(\theta)$.

Function $T(\eta)$ in equations (28)-(31) depends indirectly on the unknown function $r(\eta)$. An iterative process can be adopted, repeating successively the integration of (28) and (30) considering $T(\eta)=$ constant in the first step. The obtained solution $r(\eta)$ can then be used to calculate $T(\eta)$ for the next evaluation of (28)-(31), and the process is repeated until convergence; usually two or three iterations are enough.

The presented formulation assumes $\varphi$-independent feed and target template power patterns $U(\eta)$ and $G(\eta)$. In most cases the feed radiation pattern is not axial symmetric, but an approximation can be generated as an average of the co-components in the main planes for the lens synthesis purpose.

\subsubsection{Frequency stable radiation pattern and phase centre position}

This section presents the design of a double-shell axial symmetric lens that complies with two design conditions: a well-defined phase centre located behind the lens (outside its body) and a target far-field amplitude template (Fernandes 2010). This type of virtual 
focus lens can be useful as reflector primary feed as it will be discussed in the applications section ahead. Lens dimensions are assumed to be large relative to the wavelength, so direct GO synthesis will be used for the lens design.

Considering the geometry from Figure 20, $n_{1}$ and $n_{2}$ are the refraction indexes from the inner and outer lens shells, respectively, and $S$ and $F$ are the corresponding axial depths. The lower value of the refraction index is used at the outer shell to favour lower reflections at the air interface. The two lens surfaces are defined by the unknown functions $r_{1}(\eta), \theta(\eta)$ and $R(\eta)$ and are obtained by solving a system of three differential equations as explained next.

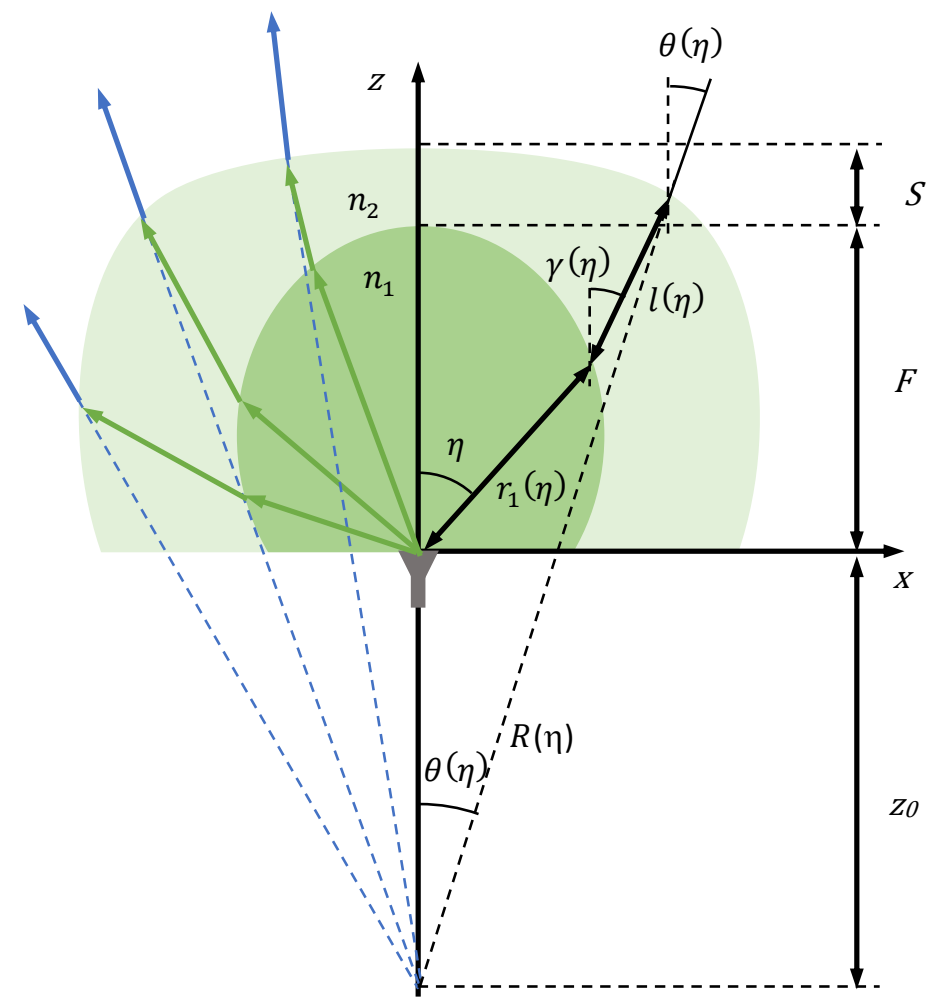

Figure 20 - Geometry for the lens design.

One of the equations derives from the power conservation condition in an elementary ray tube crossing the lens system, the same discussed in Section 2.2.3.

$$
\frac{d \theta}{d \eta}=\frac{T(\eta) U(\eta) \sin \eta}{K G(\theta) \sin \theta}
$$

where $U(\eta), G(\theta), T(\eta)$ and $K$ maintain the previous definition. Further imposing Snell law for refraction at the inner interface results in (equation (3))

$$
\frac{d r_{1}}{d \eta}=\frac{r_{1}(\eta) \sin (\gamma-\eta)}{\frac{n_{1}}{n_{2}}-\cos (\gamma-\eta)}
$$

while imposing the Snell law at the outer interface results in

$$
\frac{d R}{d \eta}=\frac{d R}{d \theta} \frac{d \theta}{d \eta}=\frac{R n_{2} \sin (\gamma-\theta)}{1-n_{2} \cos (\gamma-\theta)} \frac{T(\eta) U(\eta) \sin \eta}{K G(\theta) \sin \theta}
$$

Finally, the following path length conditions are imposed: 


$$
\left\{\begin{array}{c}
r_{1} n_{1}+l n_{2}=R \\
R \cos \theta=r_{1} \cos \eta+l \cos \gamma+z_{0}
\end{array}\right.
$$

The lens profile is obtained by solving the system of three differential equations (35), (36) and (37) with respect to $\eta$ integrating from $\eta=0$ to $\eta=\eta_{\max }$. The $\gamma$ angle required in (36) and (37) is obtained from the following explicit expression derived from (38)

$$
\gamma=\cos ^{-1}\left[\frac{n_{2}\left(R \cos \theta-r_{1} \cos \eta-z_{0}\right)}{R-n_{1} r_{1}}\right]
$$

The initial conditions at $\eta=0$ are:

$$
\left\{\begin{array}{c}
\theta=0 \\
r_{1}=F \\
\gamma=0 \\
l=S \\
R=n_{1} F+n_{2} S
\end{array}\right.
$$

The distance from the phase centre to the base of the lens $z_{0}$ is settled once the lens shells refraction index and axial depths are chosen. In fact, from (40) one has

$$
z_{0}=R(\eta=0)-F-S=F\left(n_{1}-1\right)+S\left(n_{2}-1\right)
$$

Equation (35) is numerically undetermined at $\eta=0$. To obtain its value at $\eta=0$, the following relation is written from

$$
\int_{0}^{\theta} K G(\theta) \sin \theta d \theta=\int_{0}^{\eta} T(\eta) U(\eta) \sin \eta d \eta
$$

and evaluated in the limit as $\theta \rightarrow 0$ and $\eta \rightarrow 0$. The result is

$$
\theta=\sqrt{\frac{T(\eta) U(\eta)}{K G(\theta)} \eta}
$$

where $T(\eta), U(\eta)$ and $G(\theta)$ are assumed constant near $\eta=0$ and $\theta=0$. Finally, from the above equation, the corresponding limit value for (35) is given by

$$
\frac{d \theta}{d \eta}=\sqrt{\frac{T(\eta) U(\eta)}{K G(\theta)}}
$$

This result is also used in the related part of (37) for $\eta=0$.

The GO lens synthesis is inherently broadband as long as lens dimensions are large compared to the wavelength and as long as the feed radiation pattern remains constant and coincident with the used $U(\eta)$ template.

\subsubsection{Multi-beam lens}

An integrated lens configuration, formed by two embedded shaped shells, can be designed for multi-beam or scanning applications (Costa 2008a). The possibility to shape two lens surfaces enables that two independent design goals can be specified (in a single-material lens only one design goal can be defined):

- A beam collimation condition, i.e., the output rays emerging from the lens are required to be parallel to each other; 
- A condition that minimizes aberrations of the output beam for off-axis feed positions.

The lens geometry is presented in Figure 21. The structure is axial-symmetric, formed by two embedded shells of different materials; the inner shell presents the higher refraction index value $n_{1}$ and the outer shell the lower value, $n_{2}$. An array of feeds is distributed at the planar base of the lens within a small area centred with the lens axis.

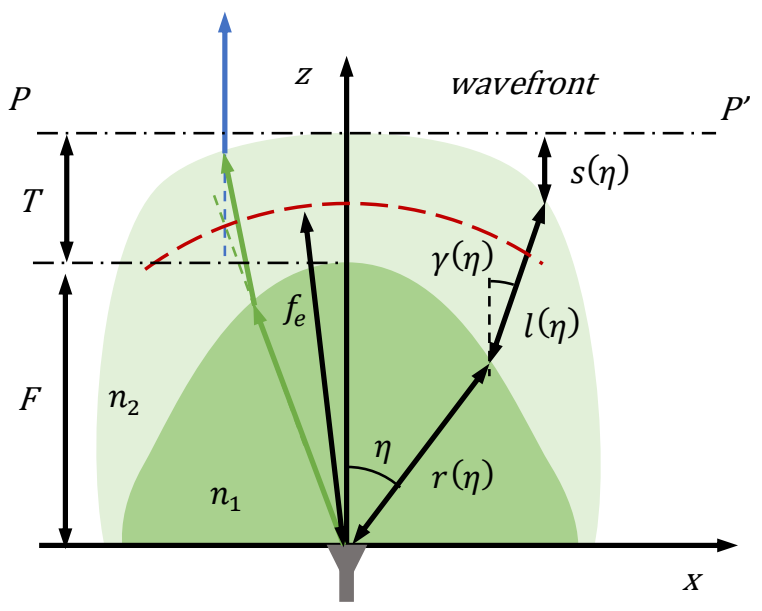

Figure $21-$ Geometry for the lens design.

The inner lens surface is defined by the (unknown) function $r(\eta)$ and the outer lens surface is defined by the (unknown) length $/(\eta)$ and angle $\gamma(\eta)$ (Figure 21 ). The lens axial thickness is $F$ and $T$ for the inner and outer shells, respectively.

It is well known that a lens satisfying the so called Abbe sine condition is free from comma aberration for a small off-axis transversal displacement of the feed (Born 1959). The Abbe sine condition is verified when the intersection points of the extended $r(\eta)$ rays departing from the on-axis feed and the corresponding extended transmitted $s(\eta)$ rays all lie over an arc of circumference with a certain radius $f_{e}$ centred at the sensor (Born 1959). This is represented by the thick dashed arc in Figure 21. In view of the geometry of Figure 21, the Abbe sine condition can be written as

$$
\left(f_{e}-r\right) \sin \eta=l \sin \gamma
$$

Snell's law at the inner interface implies that

$$
\frac{d r}{d \eta}=\frac{r(\eta) \sin (\gamma-\eta)}{\frac{n_{1}}{n_{2}}-\cos (\gamma-\eta)}
$$

In order that the optical path length of every ray is the same at the exiting wavefront, it is required that:

$$
\begin{aligned}
& n_{1} r+n_{2} l+s=n_{1} F+n_{2} T \\
& s=F+T-r \cos \eta-l \cos \gamma
\end{aligned}
$$

Equations (45)-(48) can be solved simultaneously to determine both the inner and the outer shell profiles, taking $\eta$ as the independent variable. The initial condition for $\eta=0$ is $r=F, I=T$ and $\gamma=0$ and the integration is extended up to $\eta=\eta_{e d g e}$, where generally 
$\eta_{e d g e}<\pi / 2$. The calculation is stopped at the point where the outer shell intersects the $f_{e}$ circle or where the total internal reflection condition is reached.

For a given combination of $n_{1}, n_{2}, F$ and $T$ values, the $f_{e}$ parameter can be adjusted between $F$ and $F+T$ to control the shape of the lens surfaces (and indirectly the lens scanning characteristics). In the optical limit, the lens shape obtained with the above GO-based formulation is independent of the absolute dimensions, and thus, for instance $F$, can be taken as a scaling factor.

It is noteworthy that, in line with the classical Abbe formulation for single material lenses (Born 1959), the above design equations consider only the central feed. The imposed Abbe condition implicitly determines the scanning behaviour for off-axis feeds.

\subsubsection{Beam steering lens}

In the traditional approach for mechanical beam steering, the primary feed is displaced over the focal arch of the focusing element (lens or reflector) originating a corresponding beam tilt. Alternatively the feed can be fixed and the lens (or reflector) tilted in such a way that its focal arch passes always through the feed. Phase errors in the aperture increase with the off-axis feed position originating progressive beam degradation. These become unacceptable typically beyond $25^{\circ}$ beam tilt (notable exception is the Luneburg lens, Section 2.2.1).

This section presents an alternative approach where the axis for lens tilting is coincident with the lens focus and consequently coincident with the feed phase centre, Figure 22 (Costa 2009). In this way phase errors associated with the lens tilting are eliminated and in principle larger beam scanning range can be reached, provided that proper illumination of the lens is maintained for all lens tilt angles. The feed remains stationary. An application example of this lens is presented in Section 3.2.4.

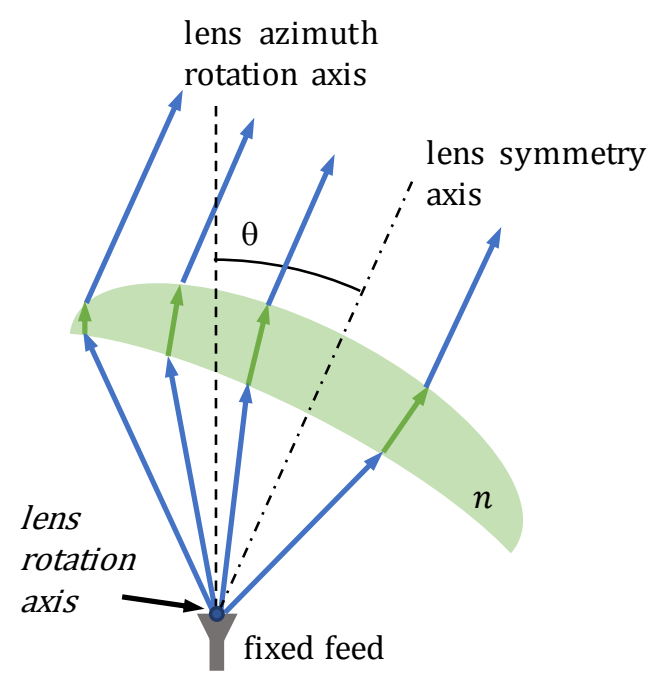

Figure 22 - Working principle of the beam steering lens.

A collimated beam lens is us ed for this concept so, by definition, all output rays emerge always parallel to the lens symmetry axis, making the $\theta$ beam tilt angle coincident with the lens tilt angle. The azimuth beam scanning is obtained by simultaneous rotation of the lens about the feed axis. 
Of course in this case the feed cannot be in contact with the lens. But the system is designed to have the feed as close to the lens base as possible (in the order of one wavelength) to favour proper lens illumination for all lens tilts. Issues to consider when designing the collimated beam lens are the maximum achievable beam tilt angle, the maximum gain and the minimum gain scan loss. These characteristics are determined by the lens profile, but they are in part limited by reflections at the dielectric interfaces and by feed illumination spill over as lens tilt increases. An appropriate feed must be designed for proper lens illumination.

This is a case where numerical optimization is required for the lens design. However, instead of brute force optimization of a spline representation of the lens, an alternative hybrid approach is considered (Section 1.3) where GO design equations are combined with a parametric representation of the lens base surface to narrow the search space. The two refraction surfaces of the lens must be designed maximizing the portion of the output lens surface that is able to collimate the feed's radiation. This is equivalent to maximize the lens $\eta_{\max }$ value defined in Figure 23 . The two lens surfaces play a role to broaden this maximum angle.

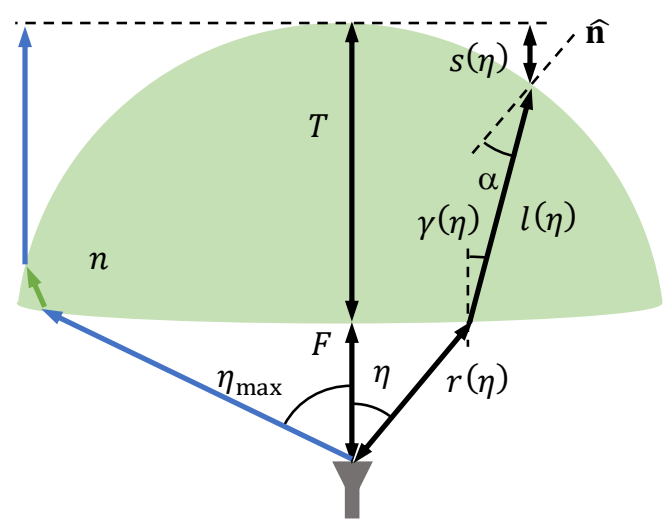

Figure 23 - Geometry for the design of the beam steering lens.

The lens geometry is shown in Figure 23. Using Snell refraction law at the bottom lens interface leads to

$$
\frac{\partial r(\eta)}{\partial \eta}=\frac{r(\eta) n \sin (\gamma-\eta)}{1-n \cos (\gamma-\eta)}
$$

where $\eta$ is the independent variable. On the other hand, by imposing an electrical path length condition, one gets

$$
r+n l+s=F+n T
$$

where

$$
s=F+T-r \cos \eta-l \cos \gamma
$$

$F$ and $T$ are input constants, whereas $r(\eta), I(\eta)$ and $\gamma(\eta)$ are unknown functions. A third design condition is required to define a unique solution. For that $r(\eta)$ is analytically written as a Taylor series expansion in $\eta$ 


$$
r(\eta)=\sum_{n=0}^{8} C_{n} \eta^{n}
$$

So the left-hand side of equation (49) can also be written analytically. The following values are set $C_{0}=F$ and $C_{1}=0$ in order to impose $\partial r / \partial \eta=0$ at $\eta=0$. This ensures null refraction for the central ray. Coefficients $C_{2}$ to $C_{8}$ are generated by using the genetic algorithm (GA) optimization method. Setting the $C_{n}$ coefficients defines the $r(\eta)$ function in $(52)$ so $\gamma(\eta)$ can be calculated from (49) and then $/(\eta)$ can be calculated from (50) and (51). The latter functions define the lens upper surface.

As mentioned, the above formulation is integrated with a GA loop to test different shapes of the bottom lens surface $r(\eta)$, with the goal to maximize the $\eta_{\max }$ angle of the lens, subject to the following constraints:

a) $r(\eta)$ must be large enough to ensure that the edges of the feed never touch the bottom lens surface when the lens is tilted;

b) The bottom lens surface cannot cross the upper surface except at the edge of the lens;

c) Ray incidence angle at the upper lens interface must be below $95 \%$ of the critical angle $\alpha_{c}$.

This latter constraint minimizes the excitation of a lateral wave (Pasqualini 2004) along the lens upper surface. This can happen when ray's incidence angle $\alpha$, measured with respect to lens local normal $\widehat{\mathbf{n}}$, approaches the total reflection condition

$$
\alpha_{c}=\operatorname{asin}(1 / n)
$$

As previously referred, the surface wave tends to deflect part of the lens radiation away from the main beam direction reducing the directivity.

\subsection{Lens analysis methods}

\subsubsection{Geometrical Optics / Physical Optics (GO/PO) analysis method}

The hybrid GO/PO method is certainly the most used approach for lens (reflector and other open structure) analysis. It takes as input the lens shape and material permittivity, the feed position, and the far-field pattern radiated by the feed when immersed in an unbounded media that has the same permittivity as the lens. The GO/PO procedure involves two steps, as implied by the acronym.

In the first step, GO formulation, as explained in Section 1.2 is used to compute the field distribution at the inner face of the lens interface; Fresnel coefficients are then used to compute the fields at the outer face of the lens. When one or more dielectric interfaces are crossed by the ray tubes originated at the feed phase centre, appropriate Fresnel coefficients and divergence factors must be used.

From the field distribution obtained in the first step, equivalent currents are calculated over the outer face of the lens, and these are Kirchhoff-Huygens (KH) integrated over the lens aperture $S$ to provide the lens far-field radiation pattern:

$$
\mathbf{E}(P)=\frac{j e^{-j k R}}{2 \lambda r} \int_{s}\left[Z\left(\widehat{\mathbf{n}} \times \mathbf{H}\left(P^{\prime}\right)\right) \times \mathbf{R}_{1}+\left(\widehat{\mathbf{n}} \times \mathbf{E}\left(P^{\prime}\right)\right)\right] \times \mathbf{R}_{1} e^{-j k \boldsymbol{\rho} \cdot \mathbf{R}_{1} d S}
$$


$\mathbf{E}\left(P^{\prime}\right)$ and $\mathbf{H}\left(P^{\prime}\right)$ represent the field produced by the feed over the lens outer surface, calculated in the first step. $\mathbf{R}_{1}$ is a unit vector directed from the origin towards the observation point $P, \rho$ is a vector directed from the origin towards the integration point $P^{\prime}$ on the lens surface, and $\widehat{\mathbf{n}}$ is the outward normal to the lens surface. This second step is referred in the literature as the Physical Optics (PO) integration.

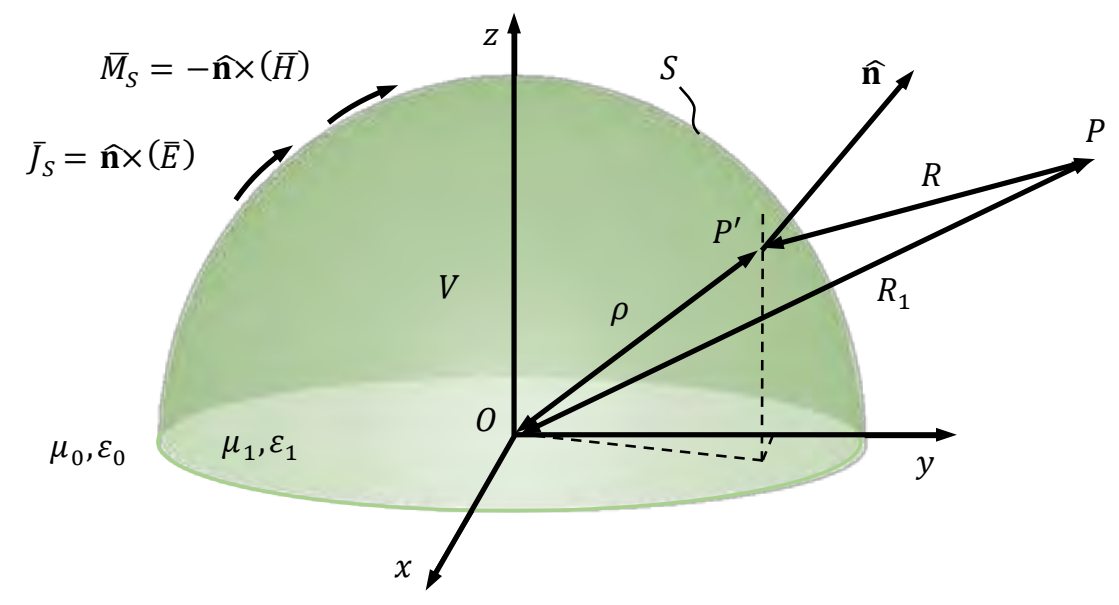

Figure 24 - Geometry for the PO aperture integration.

The GO/PO approach provides very good results for most large aperture antenna problems, provided that the accuracy of the aperture field description is also good. While in the GO direct synthesis approach discussed in the previous section it is enough to consider only the forward propagating rays to define the aperture fields (as already discussed), in the analysis process all the multiple reflected and transmitted rays must be properly accounted. Reference (Ling 1986) proposes a general procedure for tracing the rays in complex arbitrarily shaped structures in the context of radar cross-section problems, which is referred as the Shoot and Bounce Raymethod (SBR). The SBR method was used for computation of the internally reflected rays in an integrated elliptical lens (Neto 1998) or in an off-axis feed extended hemispherical lens (Pavacic 2006).

The computation time for the GO/PO method becomes really insignificant when structures are axial-symmetric. Even if the source fields are not axial-symmetric, an appropriate decomposition of those source fields in terms of a series of azimuthal harmonics may transform the non-symmetrical problem into a superposition of solutions for axial-symmetrical problems.

Memory requirements for the GO part of the procedure are reasonably modest, especially for circular-symmetric structures. A similar comment is valid in general also for the PO part of the procedure, having in mind that in some approaches the far-field pattern can be calculated as the superposition of the sequentially calculated closedform $\mathrm{KH}$-integrations of all exit ray tube fields.

\subsubsection{Physical Optics / Physical Optics (PO/PO) analysis method}

This is again a two-step analysis method, like the previously studied GO/PO, but now the calculation of the aperture fields in the first step is based on the PO formulation. This allows circumventing two $\mathrm{GO}$ limitations:

GO cannot be used in the first step for small lenses, where the feed can no longer be accurately represented by a point and by its far-field radiation pattern; 
GO fails to predict the fields near the caustics. This aspect becomes critical when caustics approach the edge of the lens affecting the correct evaluation of edge diffraction effects, or when the caustics approach the feed region thus affecting significantly the feed impedance (Neto 1998).

With the a priori knowledge of the source currents a free-space dyadic Green's function can be used to calculate the fields in an unbounded media with the same permittivity as the lens. The fields are calculated at a fictitious surface $S$ with the shape of the lens, to represent the incident field $\left(\mathbf{E}_{\text {inc }}, \mathbf{H}_{\text {inc }}\right.$ ) at a lens dielectric-to-air interface. Fresnel coefficients are used to calculate the reflected and transmitted fields at the interface, and equivalent electric and magnetic surface currents are then calculated on both sides of the interface. PO integration of the equivalent currents at the inner face of the lens allows calculating the reflected fields everywhere inside the lens. The far-field radiation pattern of the lens is determined from PO integration of the equivalent surface currents defined outside the interface from the fields obtained in the previous steps.

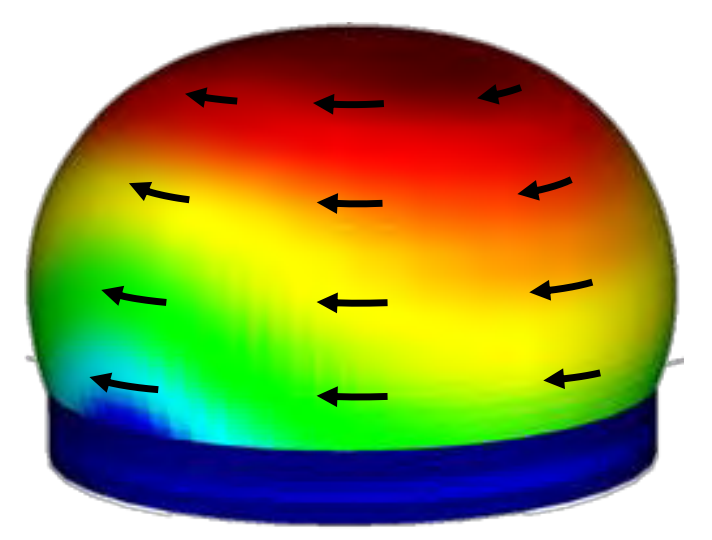

Figure 25 - Equivalent currents on the lens surface.

The asymptotic expressions that are commonly used for the PO evaluation of the farfield cannot be used in general for the evaluation of the interior fields, since the observation point may lie close to the aperture. The use of the general PO formulation valid for near-field is a little more cumbersome, and more CPU time consuming. This point aggravates for more complex structures like multiple shell lenses where more than one PO integration would have to be performed for complete evaluation of the internal fields.

This is clearly a less flexible method, and more CPU intensive than GO/PO. The insertion into an optimization loop is similar to what was discussed before for the GO/PO method, but each iteration takes longer.

\subsubsection{Spectral Domain Methods (SDM)}

Spectral Domain Methods (SDM) are a possible alternative to GO/PO for the analysis of Integrated Lens Antenna. They are particularly interesting for small lens with only a few wavelengths size where the classic Geometric Optics approach fails.

In SDM methods the lens far-field is determined from the decomposition of the feed aperture fields at the lens base into given basis functions. These functions can be, for example, plane waves or Gaussian beams (Maciel 1989). The main advantage of Gaussian beams is that they are both spatially and spectrally limited. The estimation of 
the near field at the lens base requires the use of another method, like for instance the Method of Moments. The Gaussian decomposition presents several beam parameters that can set the beam width, tilt, spatial and spectral separation between basis functions.

In (Hailu 2009; Hailu 2011) a spectral domain decomposition combined with ray tracing is used to analyse an extended hemispherical lens. Results are compared against a fullwave commercial software tool and measurements.

\subsubsection{Spherical Wave Modal Method}

The Spherical Wave Modal Method is based on the discretization of the electromagnetic field in a set of base functions, which are solutions of the wave equation in spherical coordinates. An example of this approach is found for instance in (Sanford 1994; Fuchs 2008b), for the analysis of a spherical homogenous or stratified Luneburg lens.

For each layer of the lens, the interior and exterior fields are discretized in spherical modes. A mode-matching technique is used to obtain the expansion coefficients. A priori, the only known coefficients are those of the incident wave at the exterior layer of the lens. All the other coefficients have to be determined from imposing the boundary conditions at the layer interfaces. The application of the boundary conditions creates a series of linear equations that once solved provide the coefficients of all the fields in each region.

To apply the modal method to an integrated lens antenna, the first step is to decompose the radiated field from the planar feed. First, the feed is replaced by its planar representation in terms of equivalent electric and magnetic surface currents. Then that plane is meshed and each segment is considered as an independent elementary radiator with a known discretization in spherical modes. Therefore an initial knowledge of the feed's current is needed. Since the modal method is a full-wave method, it implicitly takes into account the internal reflections at the lens interfaces.

The spherical wave mode approach is especially adequate for perfect spherical shapes. For arbitrary shaped lenses the boundary conditions of the fields are much more difficult to express in the spherical coordinate system. It is also expected that the convergence of the series becomes unacceptably slow especially when the feed off-axis distance increases.

\subsubsection{Method of Moments (MoM)}

One of the most popular methods for the full-wave analysis of arbitrary electromagnetic structures is the Method of Moments (MoM) (Peterson 1998). This method is intended for the evaluation of electric and magnetic field integral equations (EFIE and MFIE, respectively) that describe the EM problems. Those equations may be written in the form $L f=g$, where $L$ is the integral operator, $f$ the unknown and $g$ the excitation. An approximate solution may be obtained by MoM in the form

$$
f \cong \sum_{n=1}^{N} \alpha_{n} B_{n}
$$


where functions $B_{n}$ are known basis functions defined on the domain of $L$ and the scalars $\alpha_{n}$ are unknown coefficients to be determined. Equation (55) is then substituted into $L f=g$ and a system of linear equations is obtained by forcing the residual

$$
L\left(\sum_{n=1}^{N} \alpha_{n} B_{n}\right)-g=\sum_{n=1}^{N} \alpha_{n} L B_{n}-g
$$

to be orthogonal to a set of test functions $\left\{T_{1}, T_{2}, \ldots, T_{N}\right\}$. The result will be the matrix equation $L \alpha=\beta$ with the entries

$$
l_{m n}=\left\langle T_{m}, L B_{n}\right\rangle
$$

and

$$
\beta_{m}=\left\langle T_{m}, g\right\rangle
$$

where $\langle$,$\rangle represents the inner product operator. The unknown coefficients can be$ determined by inverting the matrix (Peterson 1998).

The size of this matrix may become prohibitive for large electromagnetic objects. As previously referred, lenses are usually large in terms of wavelength, so there is not much work reported in the literature about MoM analysis of dielectric lenses.

\subsubsection{Finite-Difference Time-Domain (FDTD)}

The FDTD technique utilizes a second-order central-difference scheme to approximate Maxwell's curl equations on a representative grid of discrete time and space. The electric and magnetic fields components are successively updated by repeated implementation of the finite-difference equivalents of the curl equations until the desired transient or steady-state response is achieved. This process is known as leapfrog time marching. Because the solution in the FDTD method is built up through time, it alleviates the need to form a large system of equations and thereby turns an otherwise intractable problem into one of patience.

As with MoM, the use of traditional 3D FDTD as an iterative analysis algorithm is restricted to lenses with dimensions similar to the wavelength. In fact, the number of variables of the algorithm increases cubically with the radius of the lens leading to impractical computation times for most common optimization problems. However, for structures with axial symmetry it is possible to reduce the 3D problem to a simpler 2D one and implement a faster algorithm called Body of Revolution (BOR) FDTD. BOR-FDTD can be used even if the symmetric lens is fed by an asymmetric source (Van Der Vorst 2002). In that case, the feed's current or its radiated field may be discretized into a Fourier expansion. Each element of the expansion is separately analysed by the BORFDTD algorithm, and the final result is obtained by the vector sum of all the partial BORFDTD analysis.

BOR-FDTD can be implemented for small lenses where for instance GO/PO method cannot be used. In fact, the smaller the lens the faster BOR-FDTD will converge for the solution since the number of space grid elements reduces with the size of the lens. BORFDTD can easily handle internal reflections since it is a full-wave method and can also be implemented for multiple layer or varying dielectric constant lenses. 


\subsection{Lens materials}

There are a few aspects to be taken into consideration when selecting dielectric materials for lens fabrication. Usually the materials are selected based upon their dielectric constant or relative permittivity and the usual values range from 1.2 to 11 . A second important parameter is the dielectric loss tangent $(\tan \delta)$ which measures the material dissipation loss. The lower the loss tangent value the lower the material losses per wavelength. Acceptable values of the loss tangent depend upon the application, nevertheless, in most cases a value lower than $10^{-3}$ is us ually considered adequate. It is also important to know if the dielectric materials are isotropic and homogenous. There are also mechanical considerations like material hardness, fracture toughness or melting temperature, which may be relevant for the selection of the lens fabrication technology, and thermal expansion coefficients which may be relevant for multi-shell lenses.

The electromagnetic properties of dielectric materials at millimetre wave may vary between manufacturers and even between material batches. For demanding specifications, it is advisable to accurately measure the dielectric properties of a sample of the material that will be used for the lens fabrication. Evaluation of the complex permittivity of dielectric materials usually involves vector network analyser measurement of the frequency response of a test millimetre wave circuit (it can be a resonator or not) when loaded with a dielectric sample and when empty (Chen 2005). Two classical methods are described next: the waveguide method and the open FabryPerot resonator method.

Parallelepiped samples of the dielectric material are required for the waveguide method, precisely cut with the same length of the short-circuited waveguide sampleholder and filling completely its cross section (Chen 2005). These waveguide sections operate in single-mode $\mathrm{TE}_{10}$ conditions. The complex permittivity of the material is deembedded from the measured $s_{11}$ frequency response at the open port of the sample holder, both filled with the dielectric sample and when empty (Silveirinha 2014). Two sample holders with different lengths may be used for redundant permittivity determination and detection of eventual measurement uncertainties, Figure 26.

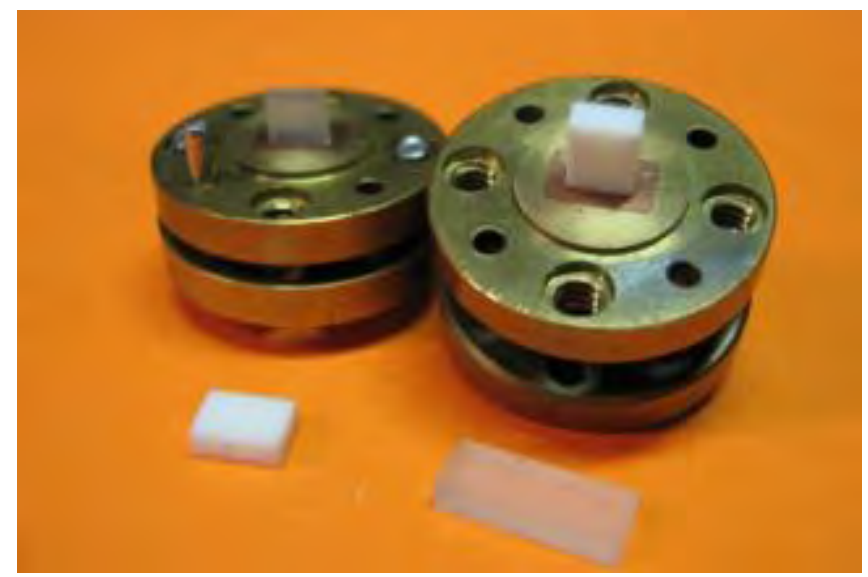

Figure 26 - Example of waveguide short-circuited sample-holder for V-band and a few dielectric samples.

One advantage of this method is that it is quite precise, provided that the air gap between the dielectric sample and the involved five walls of the waveguide sample- 
holder are negligible. Another advantage is that it requires very small samples of the material (typically $3.9 \times 1.9 \times 5.0 \mathrm{~mm}^{3}$ in the $\mathrm{V}$-band) which enables to detect material inhomogeneity or anisotropy by cutting samples from different parts and different orientations of the bulk material. Besides, it can be shown that some fortuitous measurement errors or the appearance of higher order modes can be easily perceived during the measurements, indicated by abnormal irregularities in the measured $\mathrm{s}_{11}$ frequency response (Silveirinha 2014). Another interesting point with this method is that it allows measuring loss tangents in the range up to $10^{-2}$.

The method presents however limitations for high dielectric constant values (typically larger than 6), associated with the excitation of higher-order modes in the dielectric filled sample-holder. This introduces significant error in the complex permittivity evaluation, especially in the loss factor.

The Fabry-Perot resonator method does not present the previous limitation for high permittivity samples. Its theory and operation principles are very well known (Afsar 1990; Komiyama 1991; Hirvonen 1996). There are different possible configurations for the resonator. The plane-concave configuration is commonly used since it involves only one spherical mirror and allows precise positioning of the dielectric samples at the Gaussian beam-waist without the need for perturbing holders. The material samples are typically cut as disks with a diameter typically three times larger than the Gaussian beam-waist and thickness of the order of half the wavelength in the material under test (Hirvonon 1996).

In the example shown in Figure 27 for the V-band (Fernandes 2009), the spherical mirror is made of Aluminium, with $160.3 \mathrm{~mm}$ curvature radius and $240 \mathrm{~mm}$ projected diameter. A linear translation is allowed for the planar mirror, and a $10 \mu \mathrm{m}$ precision gauge is associated with this translation. When the central distance between mirrors is around $157.3 \mathrm{~mm}$, this enables a good compromise at $63 \mathrm{GHz}$ between a narrow beam waist of the fundamental Gaussian mode at the planar mirror $\left(w_{0}=5.7 \mathrm{~mm}\right)$ and the Gaussian beam width at the spherical mirror $\left(w_{z}=42.9 \mathrm{~mm}\right)$. This choice makes it possible to measure material samples with diameters as small as $20 \mathrm{~mm}$. Increasing the distance between mirrors reduces $w_{0}$ but increases $w_{z}$ with consequent risk of spill over at the edges of the spherical mirror and consequent reduction of the cavity quality factor.

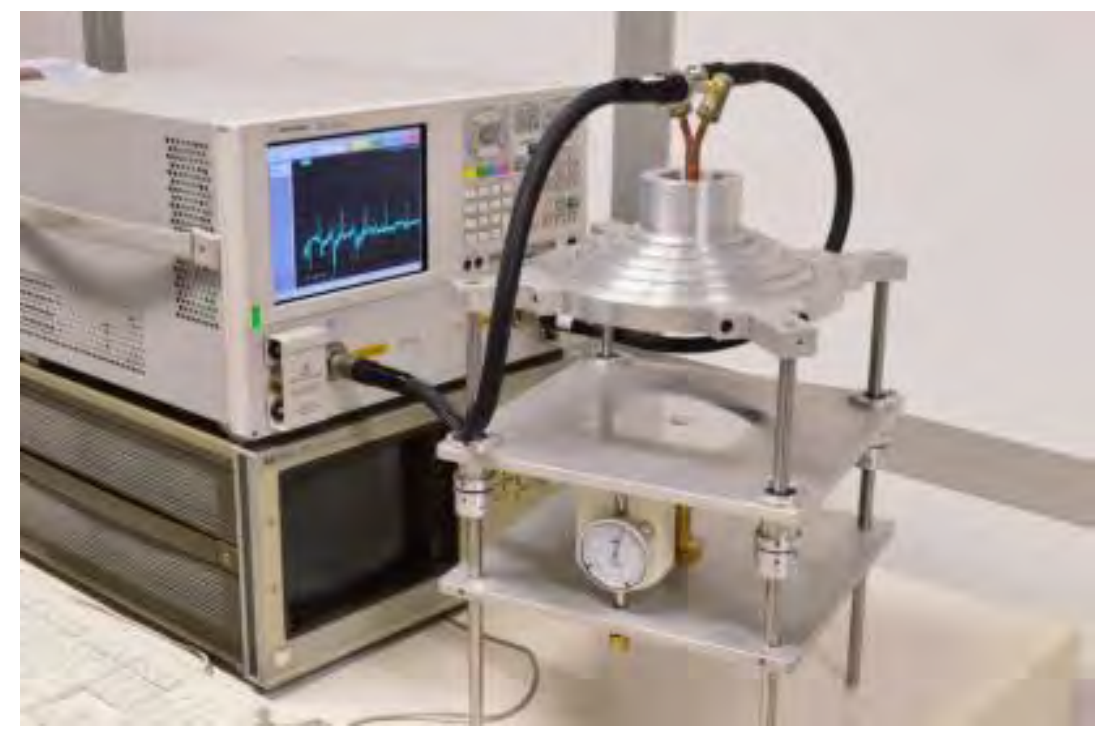


Figure 27 - Fabry-Perot open resonator for complex permittivity measurements in Vband.

Measurements can be performed using the fixed mirror distance approach. A vector network analyser sweeps the frequency within the V-band and acquires the $\mathrm{s}_{12}$ transfer function between the two waveguide ports connected to the back of the spherical mirror. In a first run the resonator is measured without any sample. Then a second measurement is performed with a dielectric disk sample placed at the centre of the plane mirror. The dielectric constant of the sample is determined from the frequency shift in the resonator modes and the loss tangent is obtained from the resonance bandwidth of a given mode (Komiyama 1991).

This method also allows evaluating the anisotropy of the material by performing successive measurements where the sample is rotated in relation to the spherical mirror axis of symmetry. In fact, since the cavity is operated with linearly polarized modes, the $\mathrm{S}_{12}$ transfer functions depend on the material anisotropy axis orientation in the disk plane (Fernandes 2009), providing different permittivity values depending on the sample rotation.

Table 3 presents a list of measured electromagnetic characteristics at $60 \mathrm{GHz}$ of some commonly used dielectric lens materials.

Table 3 - Measured permittivity values of different materials at $60 \mathrm{GHz}$.

\begin{tabular}{|l|c|c|}
\hline \multicolumn{1}{|c|}{ Material } & Dielectric Constant & Loss Tangent \\
\hline ABS-M30 (3D-printed) & 2.48 & 0.008 \\
\hline Acrylic glass & 2.5 & 0.0118 \\
\hline Alumina & 9.3 & 0.0013 \\
\hline Fused Quartz & 3.8 & 0.0015 \\
\hline MACOR & 5.5 & 0.0118 \\
\hline Polyethylene & 2.3 & 0.0003 \\
\hline Polypropylene & 2.2 & 0.0005 \\
\hline Polystyrene & 2.5 & 0.0004 \\
\hline Teflon & 2.2 & 0.0002 \\
\hline
\end{tabular}

\subsection{Lens fabrication}

Several techniques exist to manufacture a dielectric lens antenna. The most common approaches use either computer numerical control milling machine (CNC), moulding or $3 \mathrm{D}$ additive manufacturing.

CNC enables to produce physical objects from its digitized 3D description, by automatically excavating the object shape out from a block of raw material, Figure 28. This type of fabrication process is especially appropriate for antennas with complex shapes, tight dimensional tolerances and good surface finish as happens for instance with shaped lens antennas at millimetre-waves. It is more indicated for small series 
production or for lab prototyping as opposed for instance to the injection moulding process, which is cost effective for mass production. CNC milling may, however, be used for mould production for the latter technology.

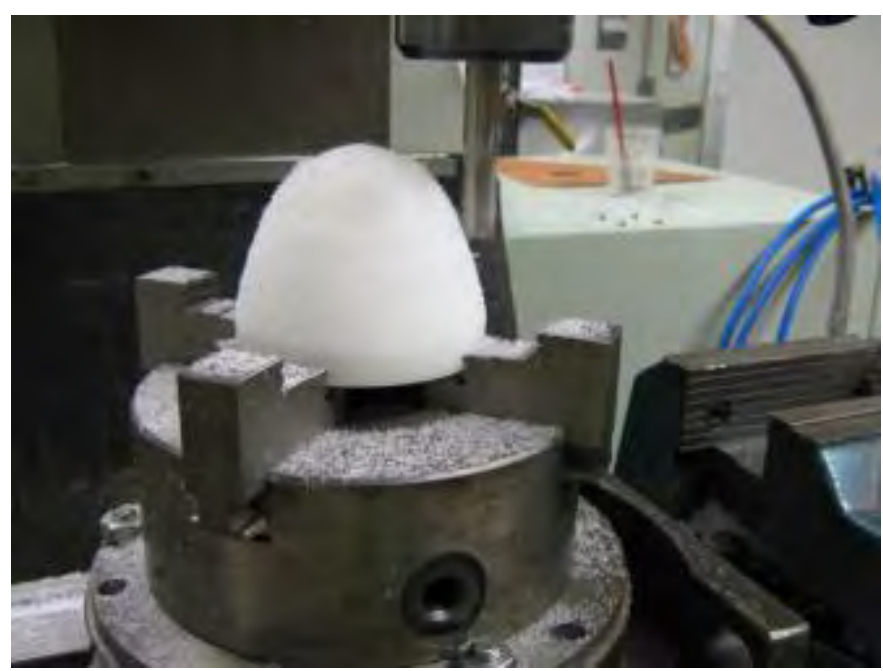

Figure 28 - Lens fabrication by CNC milling machine technique.

There are no special limitations on the materials that can be used for CNC milling, except that they must be machineable. Some examples of commercially available machineable dielectrics are listed in Table 3. The appropriate cutting tool, its rotation speed, the cutting speed, feed rate and cutting depth must be appropriate for the selected material. The specific values for these cutting parameters can be found in manufacturers' material data sheets. Depending on the dielectric material and CNC milling machine characteristics, 50 micron fabrication accuracies can be achieved. For some type of materials it is possible to fabricate the lens using a moulding technique, Figure 29. In this case a mould with the shape of the lens has to be manufactured where the dielectric, in liquid phase, is poured or injected into. Usually, the assembly must be left for a few hours before the mould can be removed. Depending on the material, the lens dimensions may change marginally after material curing; this has to be considered when designing the mould. Moulding is generally considered a cost-effective technique for mass-production, but it is of limited advantage for lens lab prototyping due to the mould manufacturing cost. Furthermore it is not easy to avoid trapped gas bubbles or to control the homogeneity of the mixture as it becomes solid, without an appropriate facility.

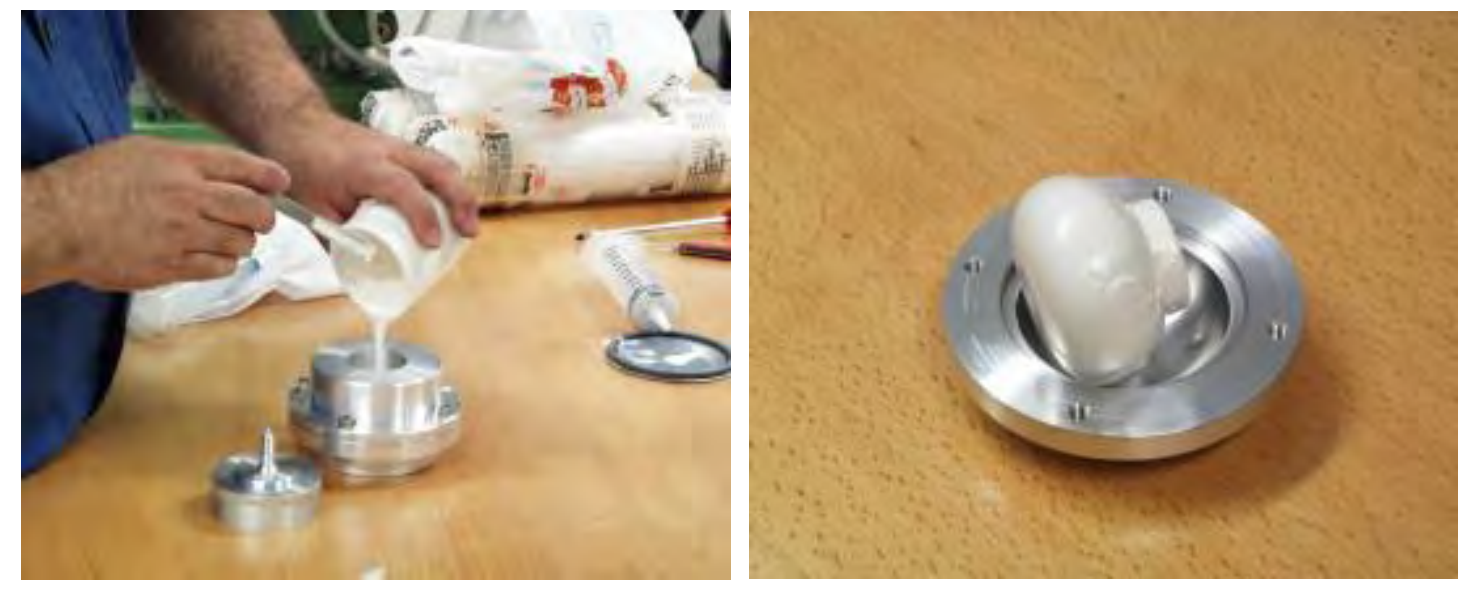


Figure 29 - Lens fabrication by moulding technique.

In the case of multilayer lenses particular attention has to be given to the matching and alignment of the layers produced by milling or moulding technologies. Air gaps between the layers should be avoided when assembling the lens. The presence of air gaps as small as 0.03 wavelengths in multi-layer lenses can reduce the antenna gain and increase side lobe level of the radiation pattern. This effect is more intense with the increase of the number of layers (Kim 1998) or with higher permittivity materials (Nguyen 2010). A glue can be used to mitigate the effect of the air gaps as long as it has approximately the same dielectric constant of one of the adjacent layers.

$3 \mathrm{D}$ printing is becoming an attractive technique to fabricate elaborate dielectric prototypes at relatively low cost. As with CNC, it starts with a 3D digitized model of the object, but the rendering is based on an additive process where successive layers of a material are laid down dot by dot to construct the desired shape. Various materials can be considered, but thermoplastic based 3D printers are the most popular. Although the commonly used materials tend to have moderately high losses and the fabrication tolerance of low-end printers is presently of the order of 200 micron, the viability of a $3 D$ printed lens antenna made of $A B S(\tan \delta=0.008$ ) for short range indoor wireless link at $60 \mathrm{GHz}$ has been recently demonstrated (Bisognin 2014). A similar technique can be used to manufacture lenses using higher permittivity lower loss materials like Alumina (Ngoc Tinh 2010).

\subsection{Lens feeds}

The main requirements for a lens feed are a reasonably uniform radiation pattern within the lens aperture subtended angle, minimum energy spill over at the lens edges and a well-defined phase centre coincident with the lens focal point. The most common families of lens feeds are either patches, slots or horns/waveguides. Patches and slots have the advantage of low profile and in some cases can be made conformal with the lens surface. However, they tend to have a broad radiation pattern which may lead to excessive spill over losses particularly in off-body fed lenses. Patches and slots are thus more appropriate for integrated lenses feeding. Waveguides, although bulky, may present some advantages for lab testing of integrated lens prototypes as they allow reusing the same feed in several lens prototypes while ensuring measurement repeatability. Horns tend to have a much more directive beam than all the previous type of feeds and are more common as off-body lens feeds.

The earlier works on integrated lens antennas at sub millimetre waves used a double slot antenna as the lens feed (Filipovic 1993; Filipovic 1997). This type of feed is particularly indicated for integration for instance with bolometers, in applications where the lens is used only as an amplitude receptor like in radio astronomy. However, the double slot antenna is narrowband and therefore limits the inherent wideband nature of the lens. Wideband feeds like the sinuous antenna (Edwards 2012), log-spiral or logperiodic antennas (Semenov 2007) have been used for radio astronomy lens applications. 


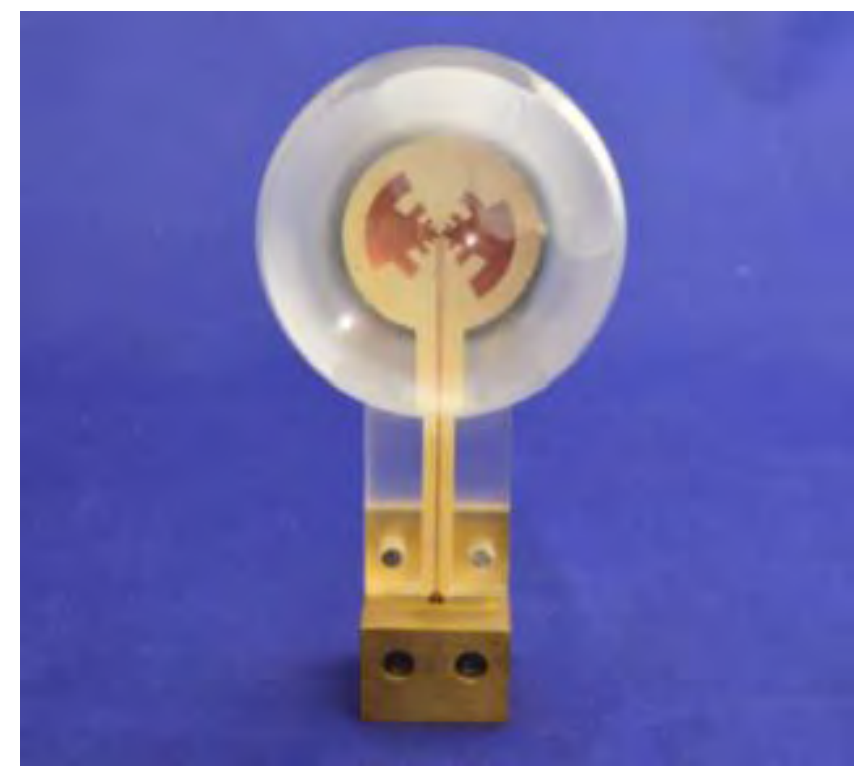

Figure 30 - Integrated lens antenna fed by a planar self-complementary log-periodic feed.

One problem with the previous wideband feeds is that the polarization is not stable with frequency. An alternative wideband planar feed that solves that problem is the crossed exponentially tapered slot (XETS) antenna (Costa 2007). It presents a frequency stable linearly polarized radiation pattern versus frequency with stable phase centre position at the centre of the antenna. The operation bandwidth is 1:3 (or 100\%). The antenna geometry is presented in Figure 31: it is formed by a single metal layer with two crossed exponentially tapered slots, intersected by a square (or star) like slot. The antenna is fed between two opposite petals, as shown in Figure 31(A), which define the antenna Eplane. The antenna perfect symmetry with respect to the feed point ensures perfect symmetry of the antenna currents with respect to the E-plane and perfect antisymmetry with respect to the $\mathrm{H}$-plane, irrespective to frequency. Consequently, pure linear polarization is obtained versus frequency at least in the main planes.

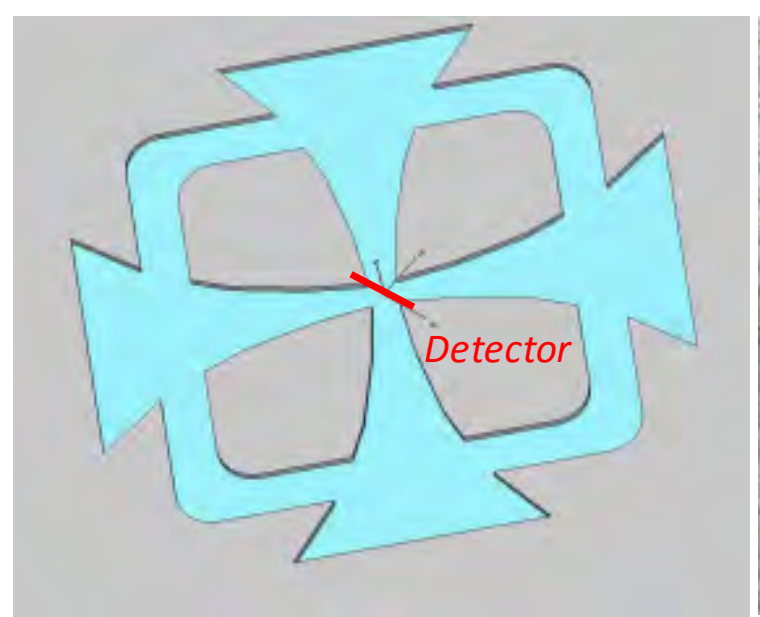

(A)

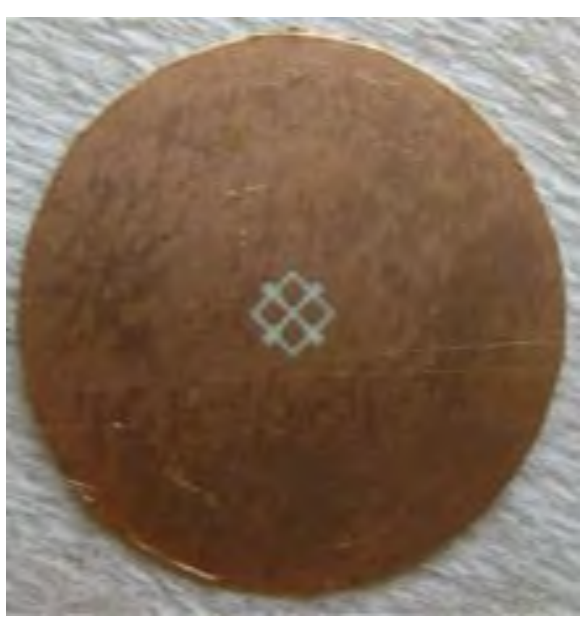

(B)

Figure 31 - (A) Configuration of a Cross Exponentially Tapered Slot (XETS) antenna; (B) prototype of the XETS for operation from 40 to $75 \mathrm{GHz}$. 
A slot feed with an extremely wideband of more than 1:10 has been presented in (Neto 2010a; Neto 2010b). It is composed by a long leaky slot line fed at the middle by an orthogonal microstrip line printed in the other face of the substrate. An air gap smaller than $1 / 16$ of the wavelength is placed between the slot and the base of the lens in order to improve the feed radiation performance. This concept has recently been extended to a square array of leaky slots (Yurdus even 2014).

All of the above single-layer antennas present a bidirectional radiation pattern due to the absence of a ground plane. This is attractive for $\mathrm{THz}$ applications to ease the feed fabrication. However, when attached to the base of a dielectric lens, most of the feed radiation is pulled into the lens body. In fact, the fraction of power that is coupled to the lens increases with the permittivity as $\varepsilon_{r}^{3 / 2}$ (Rutledge 1983). Thus, the lens material permittivity tends to be high to operate with these uniplanar feeds. Higher reflection is thus expected at the air/dielectric interface but it can be overcome with the matching layer approach or with the double-shell lens configurations discussed in Section 2.2.

Classical patch antennas are also used as integrated feeds. For example an integrated lens with an array of aperture coupled rectangular patches is feed by a microstrip feeding network at $28 \mathrm{GHz}$ (Nguyen 2011) or at $60 \mathrm{GHz}$ (Artemenko 2013a; Artemenko 2013b).

An open-end waveguide aperture is sometimes preferable for integrated lens antenna feeding, as it is easier to couple the full power to the lens without the need to increase the lens permittivity as it happens with uniplanar printed antennas. However, the standard waveguide open-end has to be modified to avoid excessive return loss and asymmetrical radiation pattern between $\mathrm{E}$ - and $\mathrm{H}$-planes. The inclusion of an edge tapered dielectric filling with the same permittivity of the lens material can mitigate the return loss problem (Ngoc Tinh 2010); however, it may originate higher order modes that affect the lens performance. To avoid this difficulty, the cross-section dimensions of the dielectric-filled waveguide must be conveniently tapered to enable single mode operation (Fernandes 2002; Fernandes 2011). When appropriately designed, the waveguide open-end itself is enough to reproduce the radiation pattern inside the lens body comparable to that of a printed or slot broadband antenna. Although the operation band of the waveguide feed may not be wide enough, the broadband characteristic of the lens can still be tested using separate waveguide feeds, each one designed for partial bands. Figure 32 presents an example of the layout and photograph of two similar waveguide feeds for Q- and V-band (Fernandes 2011). Due to the outer thread, these feeds can be easily attached or removed from the lens and reused in other lenses ensuring repeatable measurements. 


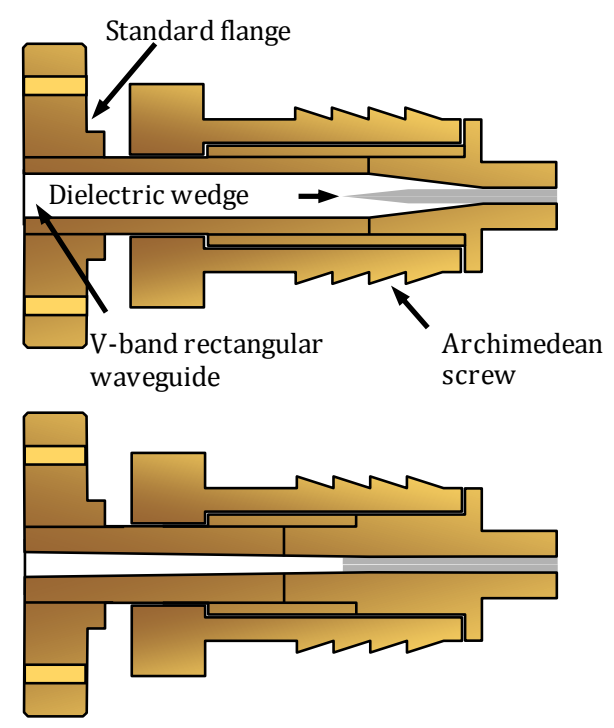

(A)

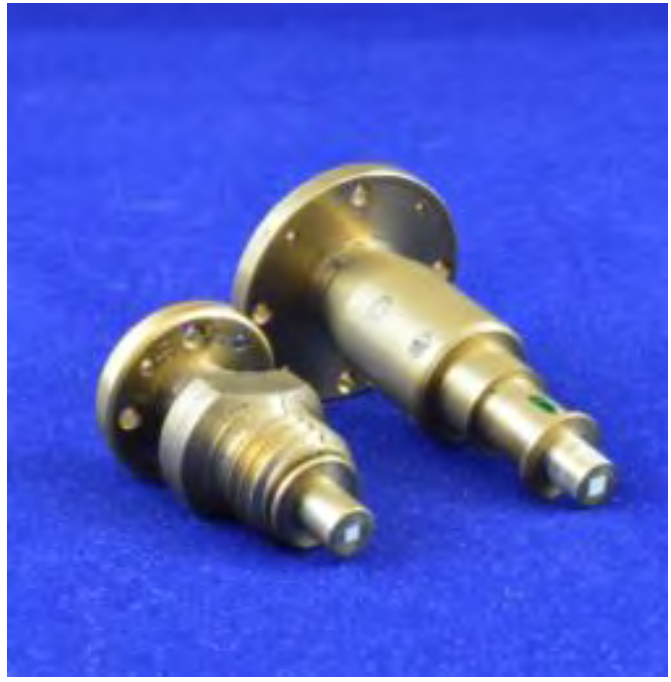

(B)

Figure $32-(A)$ Schematic view of the feed prototype (not to scale). Above: Longitudinal cut in the H-plane; Below: E-plane; (B) Photograph of the fabricated feeds. Left: for V-band operation; Right: for Q-band operation.

For off-body fed lenses it is common to use horn feeds. The requirements and the used configurations are exactly the same as for reflectors, so ample literature exists (Olver, 1994). In order to avoid excessive spill over losses usually it is required that the illumination at the edge of the lens is $10 \mathrm{~dB}$ below the power level at the centre of the lens.

\subsection{Lens measurements}

Most of the lens antenna measurement techniques are no different from those used for reflector antennas or for any other large aperture antennas. So the most common measurement procedures are not repeated here. This section addresses only two specific issues which are relevant for integrated lens antenna measurements.

\subsubsection{Feed radiation pattern in unbounded dielectric media}

Integrated lens antenna design requires the knowledge of the actual radiation pattern of the feed when it is embedded in the lens material. This is of course different from the radiation pattern of the same feed in air. Although the feed radiation pattern in unbounded dielectric medium can be obtained from some full-wave EM solvers, the direct measurement is not possible.

One practical solution that provides a reasonably good approximation is to use a spherical lens of the same dielectric material and measure at its surface the tangential fields produced by the centred embedded feed. The lens radius must be large enough to ensure the predominance of the transverse field components of the feed radiation at the lens surface. Noting that the tangential components are continuous across the dielectric-air interface and that there is no refraction in this configuration, the measured tangential fields can be considered a fair estimation of the feed radiation pattern inside the dielectric. Internal reflections may generate some ripple, but generally do not preclude the procedure. 
Figure 33 shows the application of the method for the waveguide feed presented in the previous Section. The feed is embedded in a Macor hemispherical lens and the nearfield scanning probe is also a waveguide open-end, Figure 33(A). The near-field measured result is presented in Figure 33(B), superimposed on the simulated far-field of the feed in the dielectric, computed with WIPL-D EM solver (Kolundzija 2002). The agreement is quite acceptable.

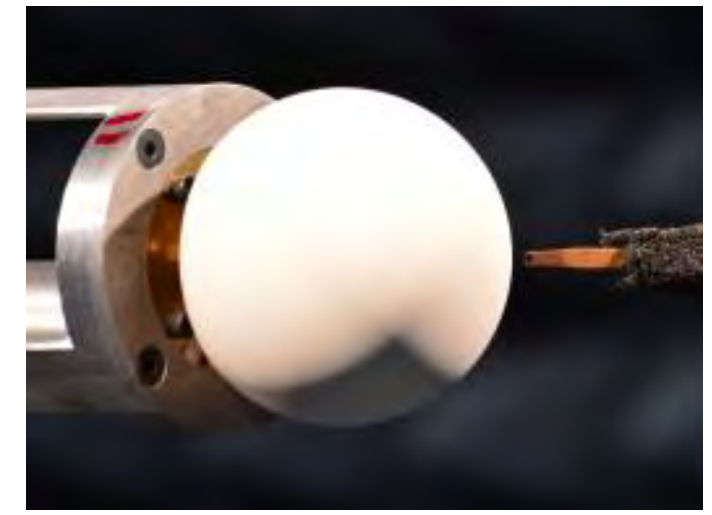

(A)

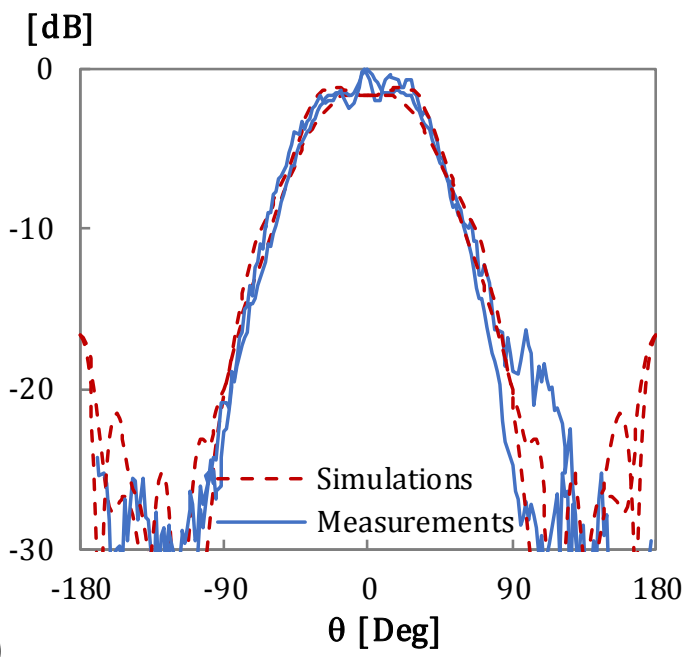

(B)

Figure 33 - Near-field scan of the tangential fields of a hemispherical Macor lens fed by a centered waveguide feed. (A) Set-up; (B) Simulated (red dashed curves) and measured (blue solid curve) $\mathrm{E}$ and $\mathrm{H}$ planes.

\subsubsection{Phase centre evaluation}

Phase centre knowledge is required when integrated lenses are used for instance as primary feeds in focusing systems. Unlike horns, where the phase center position is normally located close to the aperture plane, the phase center of integrated lenses is very dependent upon the lens shape and may even be far out from the lens body. It is a simple matter to determine it when the radiation pattern phase $\phi(\theta, \varphi)$ can be measured. Considering the geometry of Figure 34(A), it can be shown that the distance $d$ between the phase center and the rotation axis in a constant $\phi$-plane cut is given by

$$
d=-\frac{\lambda}{2 \pi} \frac{\partial \phi}{\partial(\cos \theta)}
$$

assuming that $d \ll r$. So, when the phase centre exists, $d$ is proportional to the slope of the linear variation of $\phi$ versus $\cos \theta$. This allows calculating $d$ using just a single radiation pattern measurement. 


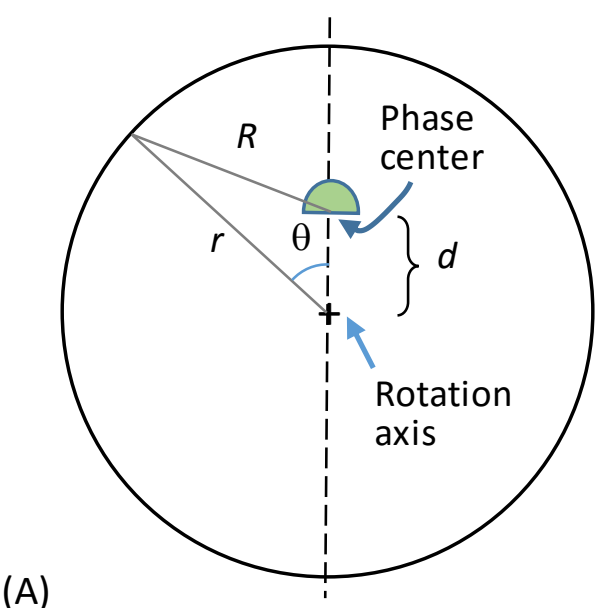

(A)

Figure $34-(A)$ Geometry for phase centre determination; (B) representation of the radiation patter phase $\phi$ versus $\cos \theta$.

However, when bolometers or other detectors are used as integrated lens feeds (Filipovic 1993), only amplitude radiation patterns can be measured, preventing the application of the previous phase centre determination method. The obvious alternative is to place the lens in the focal plane of a simple focusing system. An auxiliary convergent lens $(A C L)$ can be interposed at a fixed position between the transmitting antenna and the lens under test (LUT), while the latter is translated along the link axis until the received power is maximized. That would correspond to coincident ACL and LUT phase centres. An additional reference measurement is required with the lens replaced by an antenna with known phase centre to determine the absolute phase centre of the LUT (Costa 2010).

(A)

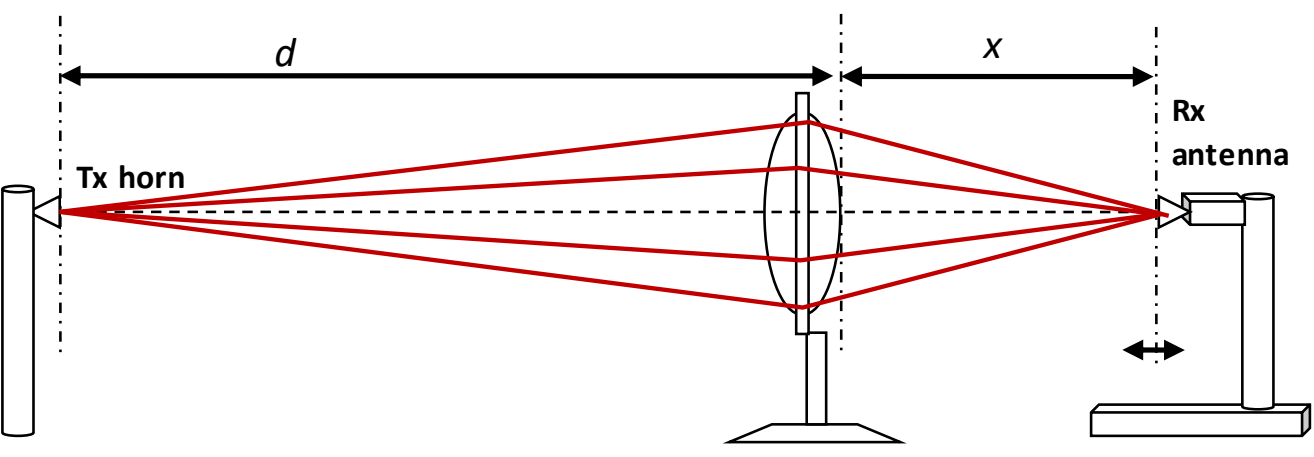

(B)

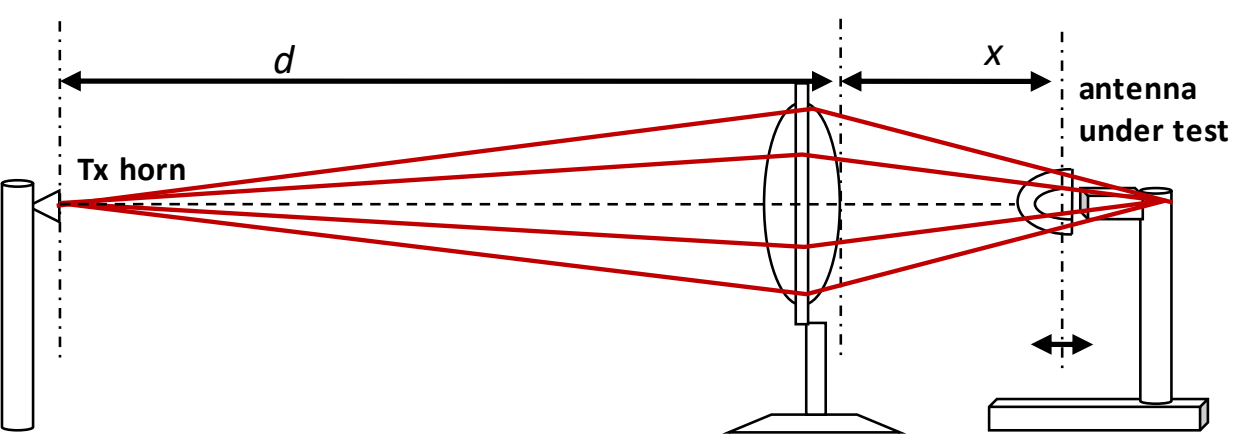

Figure 35 - Schematic set-up for phase centre determination based on power measurements: (A) Reference measurement; (B) antenna under test measurement. 
The advantage of using a lens (instead of a reflector) is that it does not need to be very large. An example is presented below where the ACL lens diameter is only three times larger than the LUT diameter, so the procedure is very convenient for small anechoic chambers. If such a small reflector was used instead, the LUT would interpose between the Tx horn and the reflector producing severe aperture blockage and consequent perturbation of the measurement.
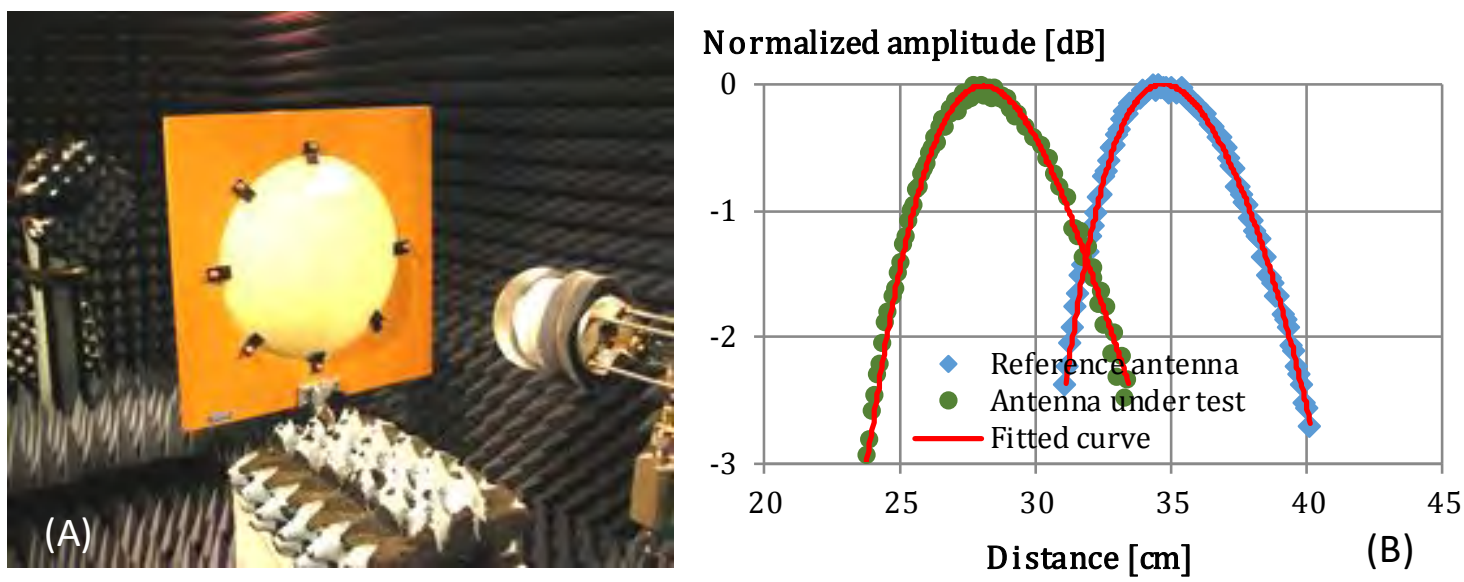

Figure 36 - Practical example. (A) Set-up; (B) Measured normalized received power vs LUT translation compared with with reference antenna translation.

Figure 36 shows an example of the set-up where the ALC, made of Teflon $\left(\varepsilon_{\mathrm{r}}=1.96 @\right.$ $62.5 \mathrm{GHz}$ ), has $300 \mathrm{~mm}$ diameter and its focal points are located $350 \mathrm{~mm}$ to the Rx side and $d=4550 \mathrm{~mm}$ on the Tx side. The LUT is a double-shell lens fed by a waveguide (Fernandes 2007) to allow verification of the amplitude-based method against the phase-based method. The reference antenna is a waveguide probe with the phase centre practically coincident with its open-end aperture. The difference between values from phase measurements and power measurements is of the order of $2 \mathrm{~mm}$ at 62.5 $\mathrm{GHz}$ which is much smaller than the depth of field of practical focusing systems at this frequency.

\section{Applications}

\subsection{Overview of applications}

This section focuses on the recent trends of dielectric lens applications, mostly involving the integrated lens configuration for microwave and millimetre wave applications were the lens size and weight are reduced. Some of the examples listed below and associated lenses are detailed in the subsequent sections.

The most common function of an integrated lens is to focus the radiation of the feed and increase the overall gain while keeping with a lower profile than what can be achieved with a reflector. This is particularly important in applications at millimetre waves like high bit rate communications at $60 \mathrm{GHz}$ (Bisognin 2014) or automotive anticollusion radar at $77 \mathrm{GHz}$ (Ka Fai 2014). Alternatively, the integrated lens can be used to shape the radiation pattern of the feed into a desired pattern. For example, for $60 \mathrm{GHz}$ indoor wireless networks it is required to have base stations with adequately shaped radiation patterns like flat-top (Ngoc Tinh 2011; Rolland 2011) or secant-squared (Fernandes 2001; Bares 2007). 
Lenses are inherently wideband so they are interesting feed candidates for some reflector focusing systems in radio telescope applications, where a wideband frequency stable radiation pattern and phase centre position are required for reflector illumination. Integrated lenses have been proposed for stable performance with either multi-layer (Fernandes 2010) or dome (Ngoc Tinh 2013) configurations. In both cases non-canonical lens shapes were obtained after proper design and/or optimization.

Lenses can be used in imaging applications, where the image of the target is focused through the lens into an array of sensors (or detectors) at the other side of the lens (Filipovic 1997). The spacing between sensors should satisfy the Nyquist sampling criterion (typically two detectors per wavelength) in order to recover the original target image. Security screening or medical imaging are examples of applications using lens antennas. For example in (Trichopoulos 2010; Trichopoulos 2013) a hemispherical lens is used with 31 by 31 detectors at its base to detect the object image. Alternatively it is possible to use an array of closely spaced lenses with one detector per lens (Llombart 2013; Naruse 2013). However, in this case the Nyquist criterion is not fulfilled. There are also some applications requiring multi-beam antennas but with a lower density of detectors like for a lens with an electrically reconfigurable beam shape (Nguyen 2011) or some degree of electrical controlled discrete beam steering (Costa 2008a; Artemenko 2013a; Artemenko 2013b).

Communications between a movable vehicle and high altitude platform or satellites requires a ground terminal antenna with a beam steerable capability. Some of the few examples in the literature that use lenses for satellite-on-the-move (SOTM) applications adopt Luneburg based configurations with mechanical beam steering capability. For example, companies like Lun'tech in France are selling commercial solutions. Alternatively, integrated lens antennas can also be used for mechanical beam steering in SOTM applications (Costa 2008b). In those configurations the inner shell of the lens is air allowing the lens moving freely above the fixed feed to steer the direction of the main beam.

\subsection{Lens application examples}

\subsubsection{Constant flux and flat-top lenses for mm-wave W-LAN}

Pioneering work on millimetre-wave cellular mobile communications started in the early nineties (Fernandes 1995) at that time aiming at $150 \mathrm{Mbit} / \mathrm{s}$ per channel. Oxygen attenuation is extremely high in this band $(15 \mathrm{~dB} / \mathrm{km})$, free-space attenuation is also very high and the same happens with obstacle attenuation. Altogether, these factors intrinsically confine radio coverage to very small cells, so unlicensed spectrum is available for emerging broadband applications.

The $60 \mathrm{GHz}$ band is recently being explored for short range multi-gigabit wireless local area networks. In indoor scenarios, Figure 37(A), the difference in free-space attenuation for different locations in the same room may represent a high dynamic range which can be compensated with an appropriately shaped antenna radiation pattern. A shaped dielectric lens antenna can be used at the base station (BST) hanging from the ceiling, that produces a secant squared $\left(\sec ^{2} \theta\right)$ type of radiation pattern in the elevation plane in order to compensate for the free space attenuation at each direction. The target radiation pattern can be expressed as 


$$
G(\theta)= \begin{cases}G\left(\theta_{\text {max }}\right) \frac{\sec (\theta)}{\sec \left(\theta_{\text {max }}\right)} & \theta \leq \theta_{\text {max }} \\ 0 & \theta>\theta_{\text {max }}\end{cases}
$$

The maximum elevation angle $\theta_{\max }$ is selected according to the desired cell radius. A sharp fall of the radiation can be imposed for $\theta>\theta_{\max }$ to conserve power and to prevent excessive reflections from the walls. An estimation of $G\left(\theta_{\max }\right)$ can be obtained from Figure $37(\mathrm{~B})$ which presents the directivity of an ideal radiation $\mathrm{sec}^{2}$ pattern vers us the maximum illumination angle $\theta_{\max }$ (Fernandes 1999a).

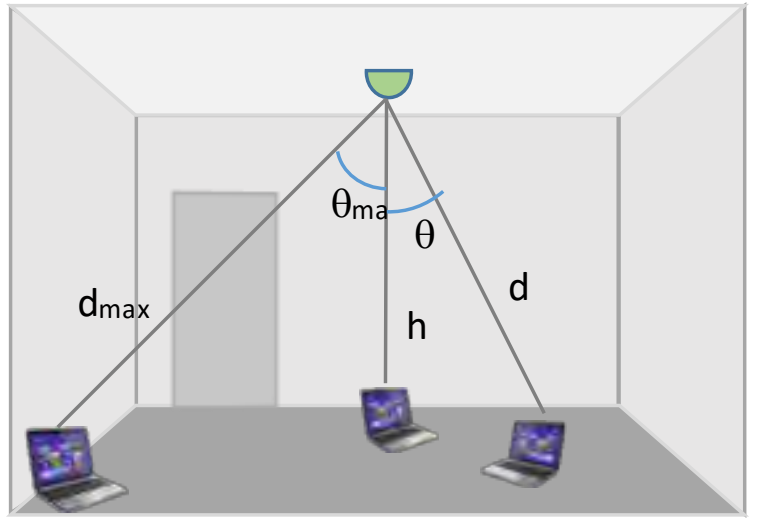

(A)

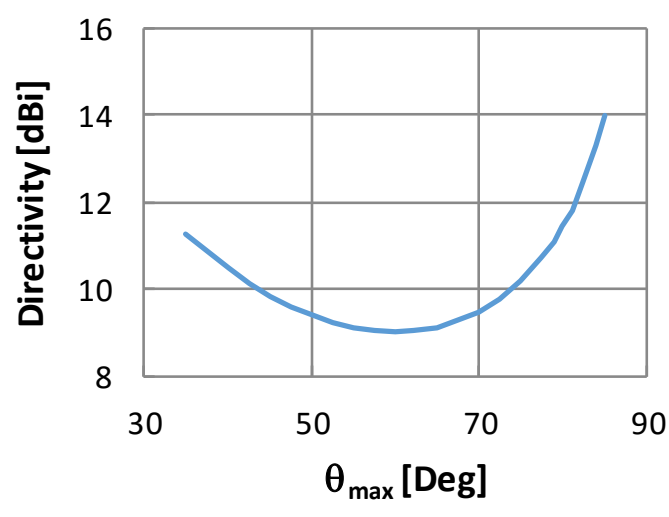

(B)

Figure 37 - (A) Millimetre-wave wireless LAN scenario; (B) Directivity of an ideal sec ${ }^{2}$ elevation radiation pattern with azimuth symmetry, versus $\theta_{\max }$.

The BST antenna must be paired with a flat-top radiation pattern antenna at the mobile terminal (MT) so that the average received power remains reasonably constant for all positions of the mobile or portable terminal within the cell. Many antenna technologies can be used to obtain such type of radiation pattern. Here a shaped lens is demonstrated, due to its possibility to reduce sharply the radiation beyond $\theta_{\max }$ and thus reduce multipath pick-up. Although the radiation patterns of the BST and MT lens antennas are circular symmetric in the present example, a square coverage footprint could also be obtained for the BST with a 3D_shaped lens, if required (Fernandes 2001).

The design of these axisymmetric amplitude shaping lenses followed the GO formulation presented in Section 2.2.3. Plexiglas material $\left(\varepsilon_{\mathrm{r}}=2.53\right.$, $\left.\tan \delta=0.012\right)$ was used for both lenses, although other lower loss commercially available materials like Polystyrene $\left(\varepsilon_{\mathrm{r}}=2.53, \tan \delta=10-4\right)$ might also be possible. Both lenses were fed by the aperture of circular metallic waveguides embedded in the lens body, carrying a circular polarized $\mathrm{TE}_{11}$ mode. The lens radiation pattern drop-out was fixed at $\theta_{\max }=75^{\circ}$ for both lenses. This corresponds roughly to $11 \mathrm{~m}$ cell radius for $\mathrm{h}=3 \mathrm{~m}$ BST antenna height with respect to MT.

Figure $38(\mathrm{~A})$ shows the fabricated prototype of the BST sec ${ }^{2}$ lens, with $66 \mathrm{~mm}$ radius. Its measured radiation pattern at $62.5 \mathrm{GHz}$ is presented in Figure 38(B). The nadir (the floor in this case) corresponds to $\theta=0$ and $\theta_{\max }=75^{\circ}$ corresponds to the maximum gain direction. 
(A)

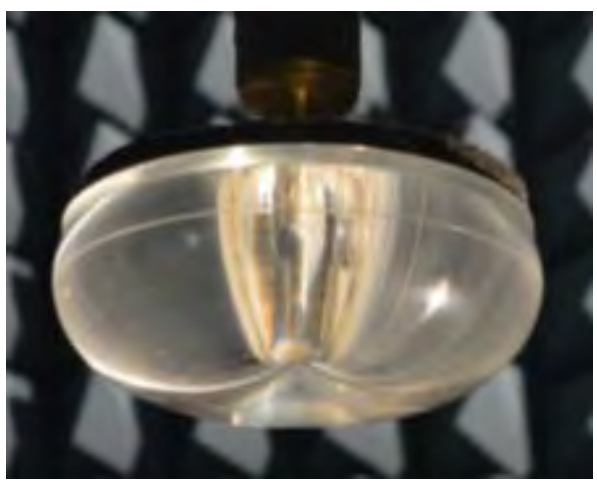

$[\mathrm{dB}]$

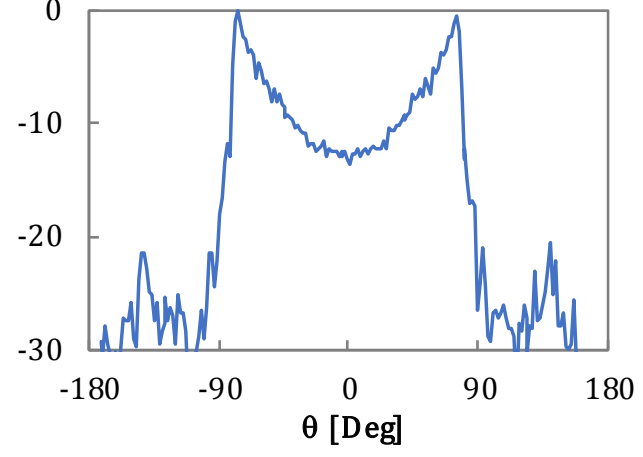

Figure $38-(A)$ Prototype of the $\sec ^{2}$ lens; (B) measured circular polarization radiation patterns at $62.5 \mathrm{GHz}$.

The fabricated flat-top lens is shown in Figure 39. In this case the lens radius is $35 \mathrm{~mm}$, considerably smaller then for the $\sec ^{2}$ lens where the sharp features of the target pattern were more pronounced. The MT elevation pattern is presented in Figure 40(B), showing a good coincidence with the desired flat-top characteristic. The zenith (ceiling in this case) corresponds to $\theta=0$. This flat top characteristic and the pattern circular symmetry favour free movement of the MT within the cell limits, and even some tilting.

(A)

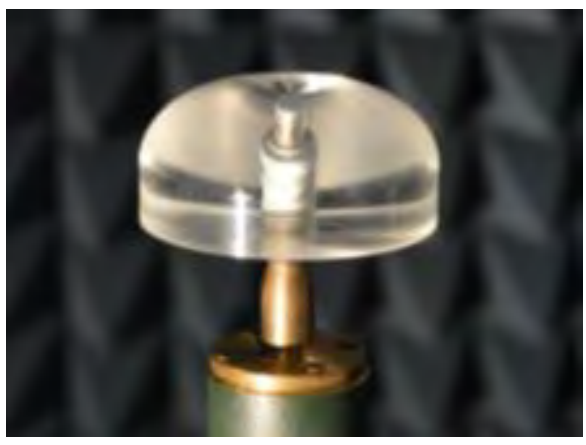

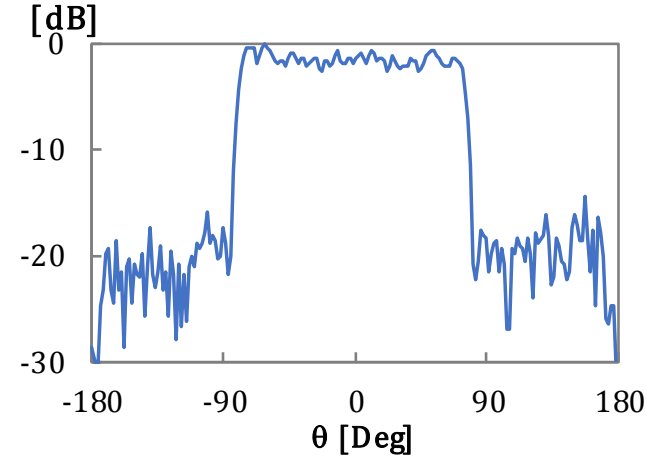

Figure 39 - (A) Prototype of the flat-top lens; (B) measured circular polarization radiation patterns at $62.5 \mathrm{GHz}$.

Considering that the link budget is affected by the product of the two antenna gain functions, this lens combination further provides very sharp cell boundaries at $\theta_{\text {max }}$, with negligible radiation outside the cell limits. A remarkable characteristic of $\mathrm{sec}^{2}$ patterns is that cell dimensions are scaled to the antenna height. This provides a simple means to control the illumination of the walls at the cell edges and maintaining an adequate compromise between multipath effects and the need for alternative paths in case of line of sight (LOS) blockage.

\subsubsection{Broadband lens for constant illumination of a reflector}

The most common reflector feeds are based on horn antennas. Despite many variants that horns may have, they all share the same feature of aperture antennas, which is a radiation pattern that increases its directivity with the frequency. When large bandwidths are involved, this may represent an important reduction of the reflector aperture illumination efficiency. This problem is often circumvented by splitting the 
wide band into multiple sub-bands, each with its own feed horn; but then a complex setup is required to minimize aberrations due to off-axis positioning of the multiple horns.

A challenging specification for a reflector feed is the ability to maintain a constant radiation pattern beam width with stable phase centre over a 1:3 bandwidth. A doubleshell shaped dielectric lens can be designed to meet such specification using the formulation from section 2.2.4 (Fernandes 2010). The lens is intended to feed a $90^{\circ}$ offset reflector. The lens output beam produces a virtual focus located far below the lens and close to the corresponding reflector focal point, Figure 40(A).

(A)
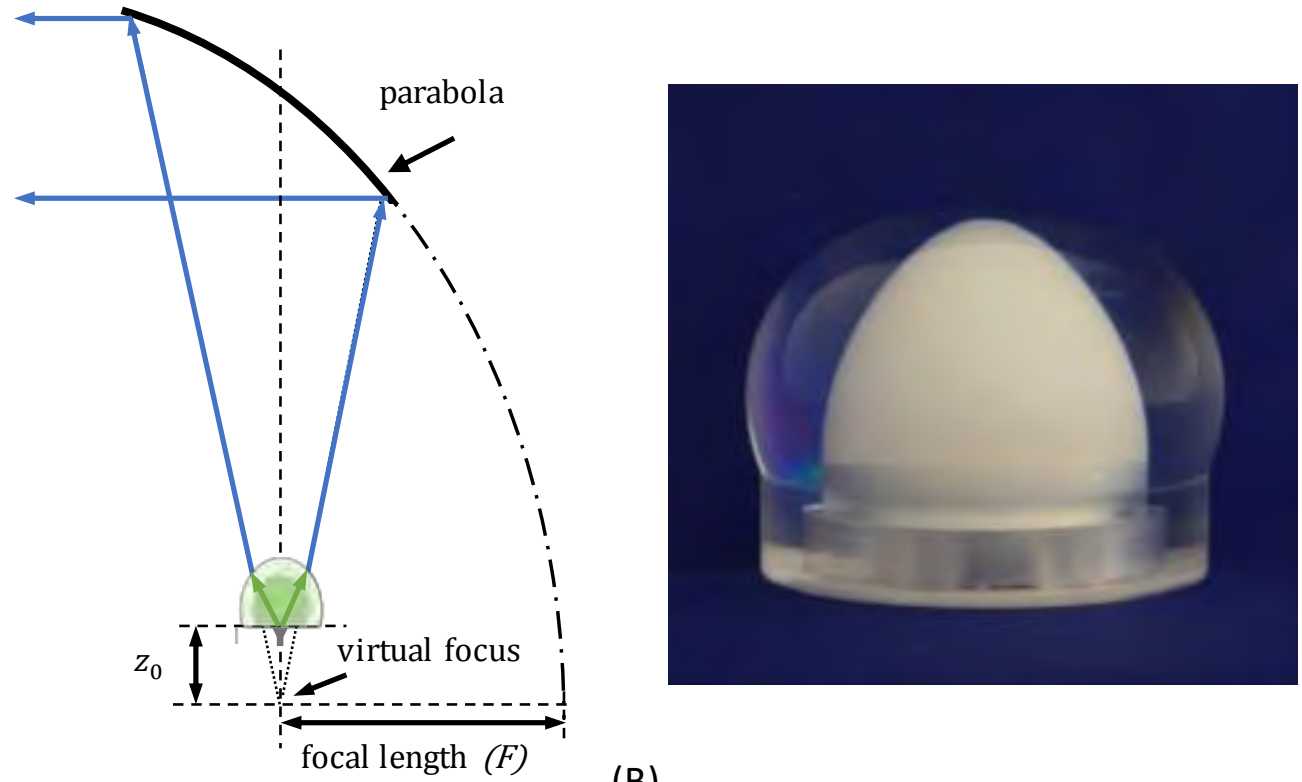

(B)

Figure $40-(A)$ problem geometry; (B) fabricated lens prototype with a MACOR ${ }^{\mathrm{TM}}$ inner shell and an Acrylic outer shell.

A $20-\lambda$ diameter lens prototype was designed and fabricated using MACOR and acrylic (Figure 40(B)). The optical transparency of the acrylic reveals the inner shell, which appears distorted due to refraction effects. The lens is fed by the waveguide device described in Section 2.6 (Figure 32). The measured lens radiation patterns are shown in Figure $41(\mathrm{~A})$ for two distinct frequencies in the Q- and V-band $(40 \mathrm{GHz}$ and $62.5 \mathrm{GHz})$. The measured lens radiation pattern agrees very well with the Gaussian template with $\alpha_{0}=23^{\circ}$ Gaussian width (dashed curve). It is possible to see that the shape and beam width of the lens radiation pattern are identical in both bands as desired. The same behaviour was demonstrated by full-wave simulation between $30 \mathrm{GHz}$ and $90 \mathrm{GHz}$ (Fernandes 2010). The lens output beam Gaussicity is better than $94 \%$ over the band. The measured phase centre position of the lens agrees with the value imposed during the design $\left(z_{0}=68.7 \mathrm{~mm}\right)$ and it is identical in both frequencies. Full-wave simulations confirm phase centre stability over the 1:3 bandwidth.

The performance of the lens-reflector assembly was simulated using the ILASH software (Lima 2008). Figure 41(B) shows that the aperture efficiency is practically constant versus frequency as required and, in agreement with that property, the reflector directivity in $\mathrm{dB}$ increases almost linearly with logarithm of the frequency. 

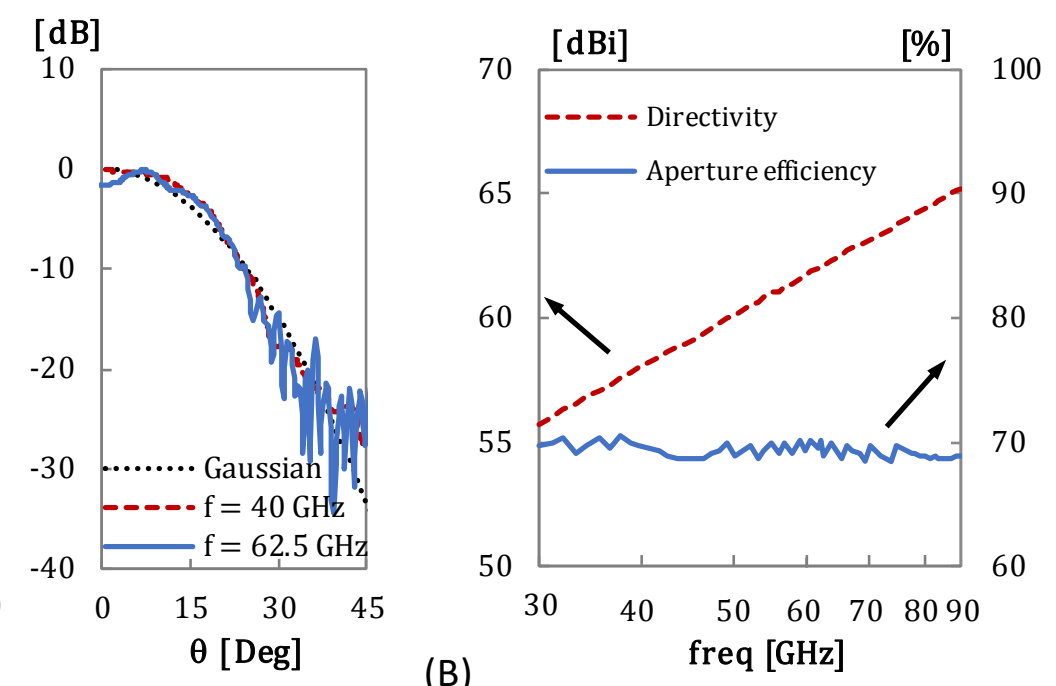

(A)

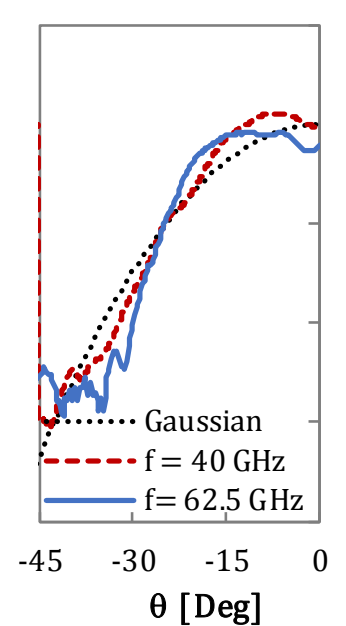

Figure $41-(\mathrm{A})$ Measured radiation patterns of the lens at $40 \mathrm{GHz}$ and $62.5 \mathrm{GHz}$; (B) calculated directivity and aperture efficiency of the reflector with frequency plotted on log scale.

\subsubsection{Multi-beam lens}

Two examples of compact integrated lens antennas are presented here for multi-beam applications operating over a $40 \%$ bandwidth from $40 \mathrm{GHz}$ to $60 \mathrm{GHz}$. The first example is intended to demonstrate the potential of a double-shell lens to produce eleven beams within $\pm 20^{\circ}$ scan interval with $6^{\circ}$ beam width at $60 \mathrm{GHz}$, gain scan loss lower than $1 \mathrm{~dB}$ and better than $95 \%$ Gaussissity throughout the $40 \%$ bandwidth. In this example the lens is fed by a waveguide. The main purpose of the second example is to demonstrate the viability of the broadband XETS antenna introduced in Section 2.6 as an effective uniplanar linear polarization lens feed for multi-beam applications. Its configuration allows very tight antenna packing at the lens base for proper adjacent beam overlap. The tight packing does not preclude the integration of each XETS element with its Schottky diode for operation as a mixer. A canonical integrated lens is used for this feed demonstration.

The scarce integrated lens configurations reported in the literature for scanning or multibeam applications are based only on extended hemispherical or elliptical lens configurations (Filipovic 1997; Wu 2001). In these canonical structures the lens shape is fixed, and so the available degree of freedom for design is just the feed distance to the lens radiating surface which does not allow enforcing any condition to reduce the aperture phase errors for off-axis feed positions. A double-shell configuration was adopted in this example to allow imposing the Abbe sine condition. The selected lens materials were the MACOR ${ }^{\mathrm{TM}}$ /acrylic combination (permittivity 5.5/2.53), and the selected feed was the waveguide device described in section 2.6 for $40 \mathrm{GHz}$ and 62.5 $\mathrm{GHz}$ operation, see Figure 32.

Figure 42(A) shows the fabricated lens antenna prototype (Costa 2008a) which was designed using the GO formulation from Section 2.2.5 implemented in the ILASH software (Lima 2008). The lens dimensions are $60 \mathrm{~mm}$ diameter at the base and $37 \mathrm{~mm}$ height. Figure 42(B) shows the measured and simulated radiation patterns for different feed positions at $1.1 \mathrm{~mm}$ steps along the lens base in the $\mathrm{H}$-plane. This produces 
overlapping of consecutive beams near $-1.5 \mathrm{~dB}$ level with respect to maximum. Beam overlap at $-3 \mathrm{~dB}$ occurs for wider separation between consecutive feeds, compatible with the used feed aperture size (not shown). The figure shows excellent agreement between ILASH predictions and measurements and confirms the effectiveness of the proposed double-shell lens concept regarding beam scan linearity, internal reflections, beam shape and Gaussissity preservation with extremely low scan loss up to $20^{\circ}$ elevation. The achieved gain was of the order of $18-19 \mathrm{dBi}$ and the scan loss better than $1 \mathrm{~dB}$ for 43 to $62 \mathrm{GHz}$. As a consequence of the GO-based lens design, the scan angle dependence on the feed off-axis position was practically independent of frequency and the beam Gaussissity was better than $95 \%$.

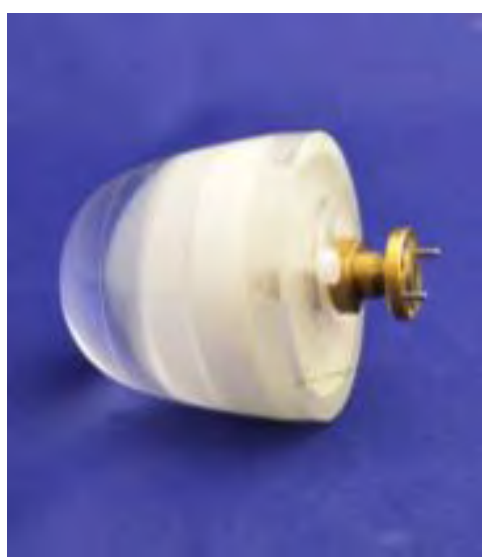

(A)

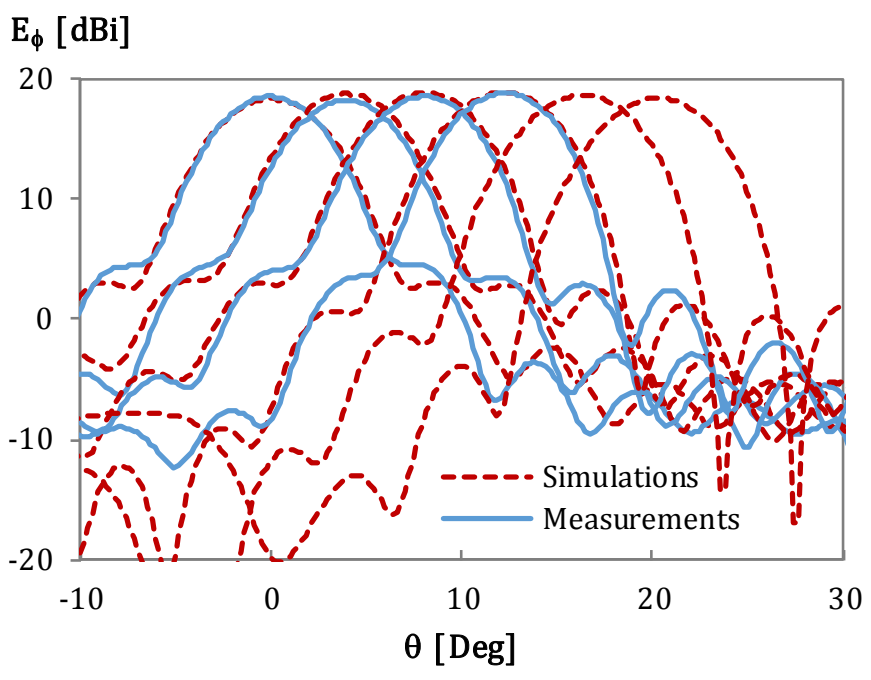

$\theta[\operatorname{Deg}]$

(B)

Figure 42 - (A) fabricated MACOR ${ }^{\mathrm{TM}} /$ acrylic lens prototype, showing the attached 60$\mathrm{GHz}$ band waveguide feed; (B) simulated and measured gain radiation patterns at 62.5 $\mathrm{GHz}$, in the $\mathrm{H}$-plane, with feed at $0,1.1,2.2,3.3,4.4$, and $5.5 \mathrm{~mm}$ from the lens axis.

The following example is intended to demonstrate the viability of the wideband XETS printed antenna with simple integral mixer described in section 2.6 as an integrated multi-beam lens feed and to show the effectiveness of the IF signal retrieval setup. Despite the tightly packed assembly of the XETS elements at the lens base, the proposed feed still offers good isolation between adjacent elements both at RF and IF, providing very stable radiation pattern and linear polarization over the bandwidth.

The above objectives can be demonstrated using a canonical single material lens, the conclusions remaining valid for more sophisticated lens designs. A $68 \mathrm{~mm}$ diameter MACOR elliptical lens was used in this case (Costa 2007b), Figure 43(A). The lens was designed to scan the angular interval from 0 to $\pm 18^{\circ}$ with beam overlap approximately at $-3 \mathrm{~dB}$ for $43 \mathrm{GHz}$ and at $-4.5 \mathrm{~dB}$ for $62.5 \mathrm{GHz}$. The overlap level at the higher frequencies could have been improved with other lens designs, but this type of optimization exceeded the established objective for this example.

The radiation pattern measurement configuration is shown in Figure 43(B). The Schottky diode integrated into the XETS receives both the $62.5 \mathrm{GHz}$ far-field Tx signal (through the lens) and the $64 \mathrm{GHz}$ LO signal (through the airfrom the back), producing the desired 1.5 GHz IF signal. The LO assembly is fixed with respect to the lens, so that the LO signal 
amplitude illuminating the XETS does not change with lens rotation, whereas the IF signal amplitude changes only in response to lens radiation pattern at the TX frequency.

(A)
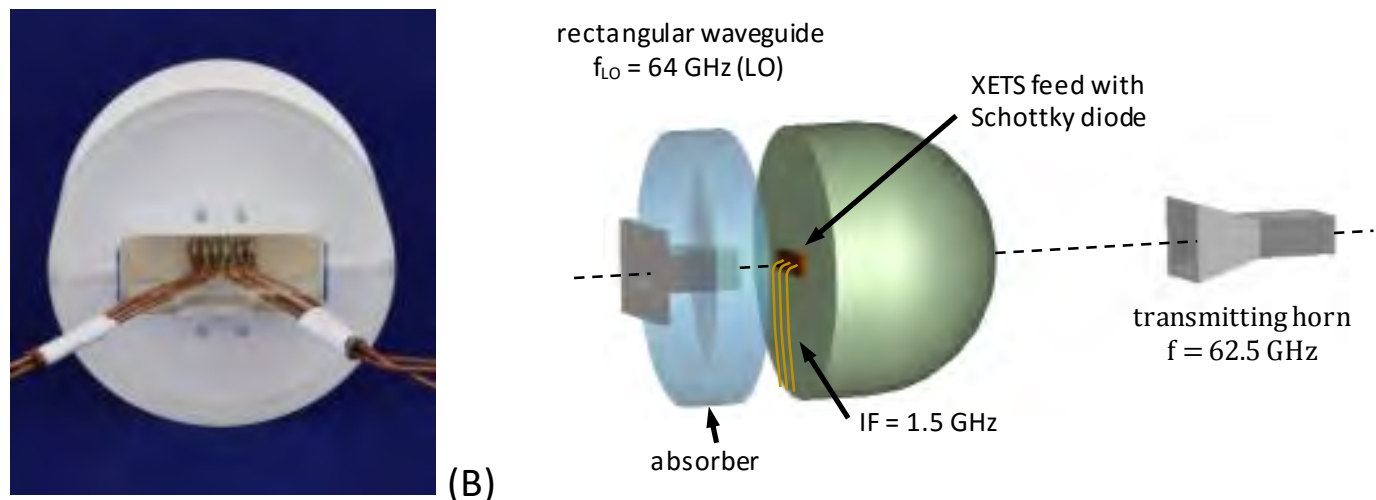

Figure $43-(A)$ bottom view of a MACOR elliptical lens fed by a multi-XETS assembly with an integrated mixer; (B) radiation pattern measurement setup at $62.5 \mathrm{GHz}$, using an integrated mixer at the base of the lens.

Examples of the $\mathrm{H}$-plane measured and simulated radiation patterns are depicted in Figure 44 at $62.5 \mathrm{GHz}$ for three XETS elements (\#2, \#3 and \#6). The curves are normalized to the respective maximum. The main lobe shape is quite stable, with the correct beam inclination despite the very close position of the XETS elements. Cross-polar level is below $-15 \mathrm{~dB}$, which is much better than what can be obtained for instance with the selfcomplementary log-periodic feed. This validates the expected feed performance.

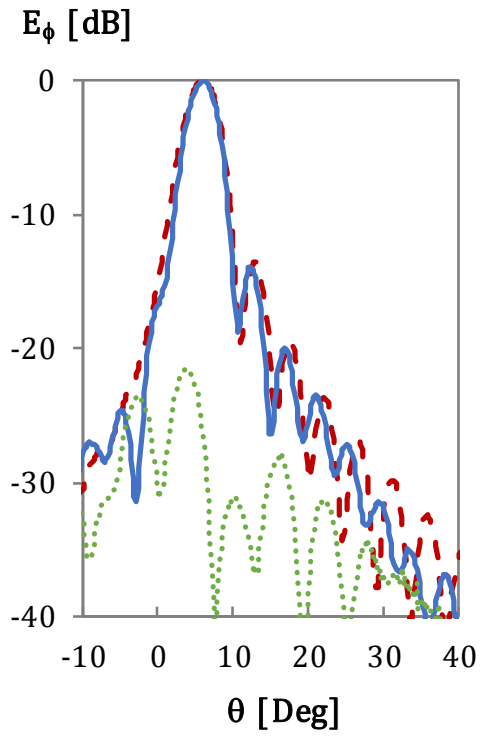

(A)

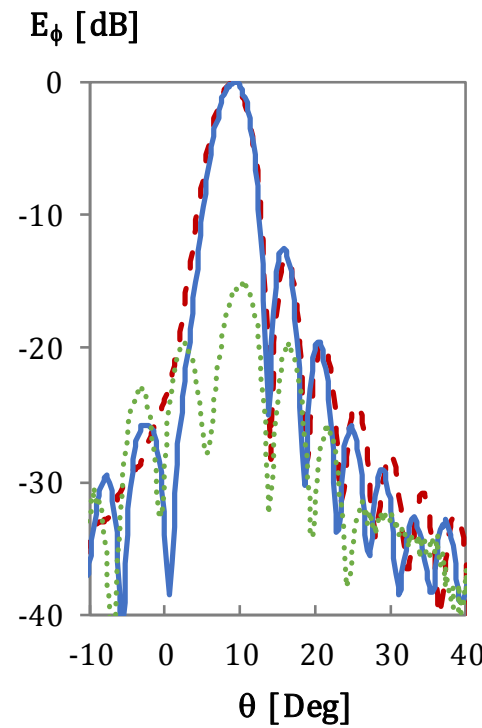

(B)

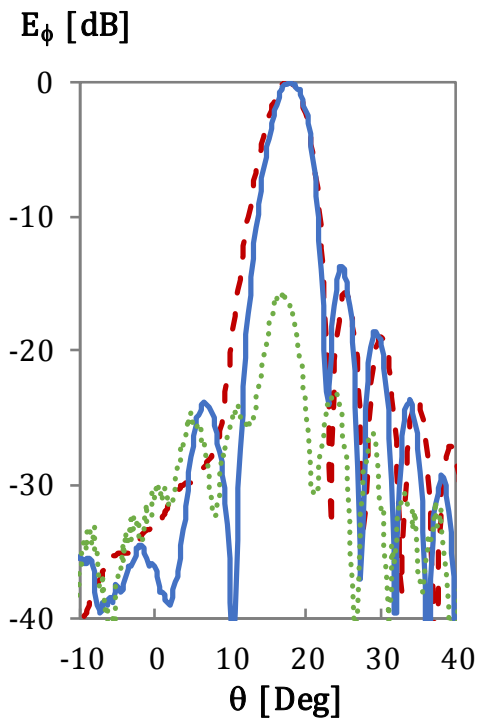

(C)

Figure 44 - Comparison between simulated and measurements radiation pattern at

$62.5 \mathrm{GHz}$ in the $\mathrm{H}$-plane (red dashed line - simulations Co-pol, blue line measurements Co-pol and green dotted line-measurements $X$-pol) for a feed displacement of: (A) $x=2 \mathrm{~mm}$; (B) $x=3 \mathrm{~mm}$; (C) $x=6 \mathrm{~mm}$.

\subsubsection{Mechanical beam-steering lenses}

Mechanical beam steering antennas tend to be superseded by electronic beam steering antennas because the latter usually offer more compact and flexible solutions. But at 
millimetre-waves this trend is not so clear as losses and cost of phased arrays increase considerably. The main drawbacks of the mechanical beam steering solutions are the mechanical complexity and the expensive millimetre-wave rotary joint.

A new approach for mechanical beam steering has been proposed in (Costa 2008b; Costa 2009) where the feed remains stationary while the beam tilt is obtained just by rotating or tilting an appropriately shaped dielectric lens that is held by a very simple mechanical structure in the near-field of the fixed feed aperture.

One example of this configuration was intended for Low Earth Orbit satellite telemetry link at $26 \mathrm{GHz}$. The lens was required to produce a sector beam with shaping in elevation, and to enable a simple mechanical azimuth scanning. The target gain versus elevation angle $\theta$ is given approximately by $\operatorname{Sec}(k \theta)$. With an appropriate choice of the $k$ shaping parameter, this elevation radiation pattern compensates path loss attenuation taking into account the Earth curvature.

The designed shaped lens is axial-symmetric, but a spherical air cavity is excavated through its base at a calculated off-set distance with respect to the lens symmetry axis, Figure 45(A). The feed aperture is at the centre of this off-set spherical air cavity. The asymmetry introduced by the feed position produces an adequate directive beam in the azimuth plane with $\operatorname{Sec}(k \theta)$ shaping in elevation. This is an extremely simple solution where the feed is fixed and the lens rotates about the eccentric feed axis to provide the required beam scanning. This contactless feeding avoids the need for fault prone rotary joints in long continuous usage. The assembly is very compact and lightweight (lens diameter is less than 5 wavelengths and height is less than 3 wavelengths) complying with mass and volume restrictions for satellites.

(A)

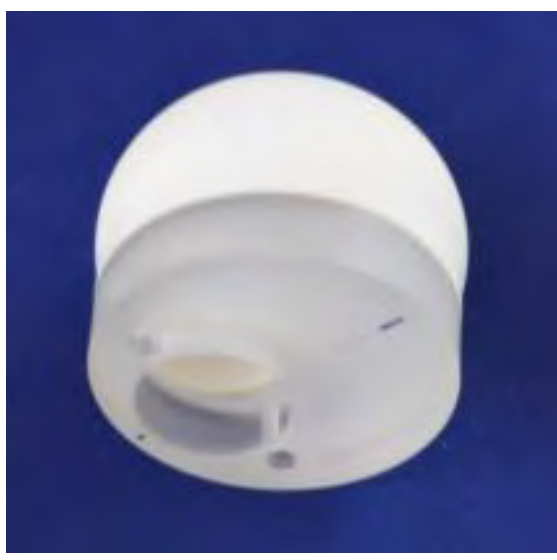

(B)

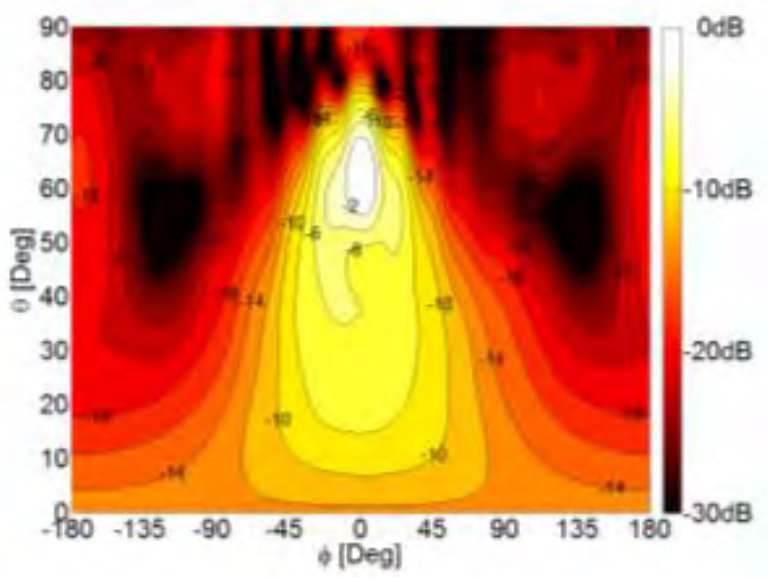

Figure $45-(\mathrm{A}) \mathrm{MACOR}^{\mathrm{TM}}$ axial symmetric $50 \mathrm{~mm}$ diameter shaped lens; (B) radiation performance of the lens computed with the GO/PO method.

The same concept of a moving lens with fixed feed was extended for azimuth plus elevation mechanical scanning in a different application context (Costa 2009). Wireless indoor transmission of uncompressed HD video signal between a video device like a camcorder and a HD TV Display may soon become a requirement for mass market home applications. Emerging standards are proposing to use the unlicensed spectrum from 57 $\mathrm{GHz}$ to $66 \mathrm{GHz}$ for such applications. For mass consumer applications, the radio link must use inexpensive low power sources and hence high gain antennas ( $>20 \mathrm{dBi}$ ) are required to favour the link budget. The narrow beam needs to be steered to allow for the user 
mobility. Using the design formulation described in Section 2.2.6, a new configuration of a mechanical steerable beam antenna has been developed where a dielectric lens pivots in front of a single stationary moderate gain feed (Figure 46(A)). Again, the feed is not in physical contact with the lens. As previously discussed, this eliminates the need for rotary joints and the associated drawbacks. The designed lens is made of polyethylene and is such that both the input and output lens surfaces are shaped to accommodate a beam collimation condition and a scan angle maximization condition. The lens output beam is aligned with the lens axis for all lens orientations within the operation cone. Thus, pivoting the lens around two of its main axis allows steering the beam in elevation and azimuth. The important difference with respect to conventional scanning lenses is that for all lens inclinations the focus is always exactly coincident with the aperture phase centre. In this way, aberrations are strongly reduced allowing wider scan angles than conventional solutions. The fabricated lens assembly demonstrated a $45^{\circ}$ to $+45^{\circ}$ elevation scan capability over full azimuth, with $21 \mathrm{~dB}$ gain and better than $-1.1 \mathrm{~dB}$ gain scan loss (Figure 46(B)). The radiation efficiency is always above $95 \%$.

(A)
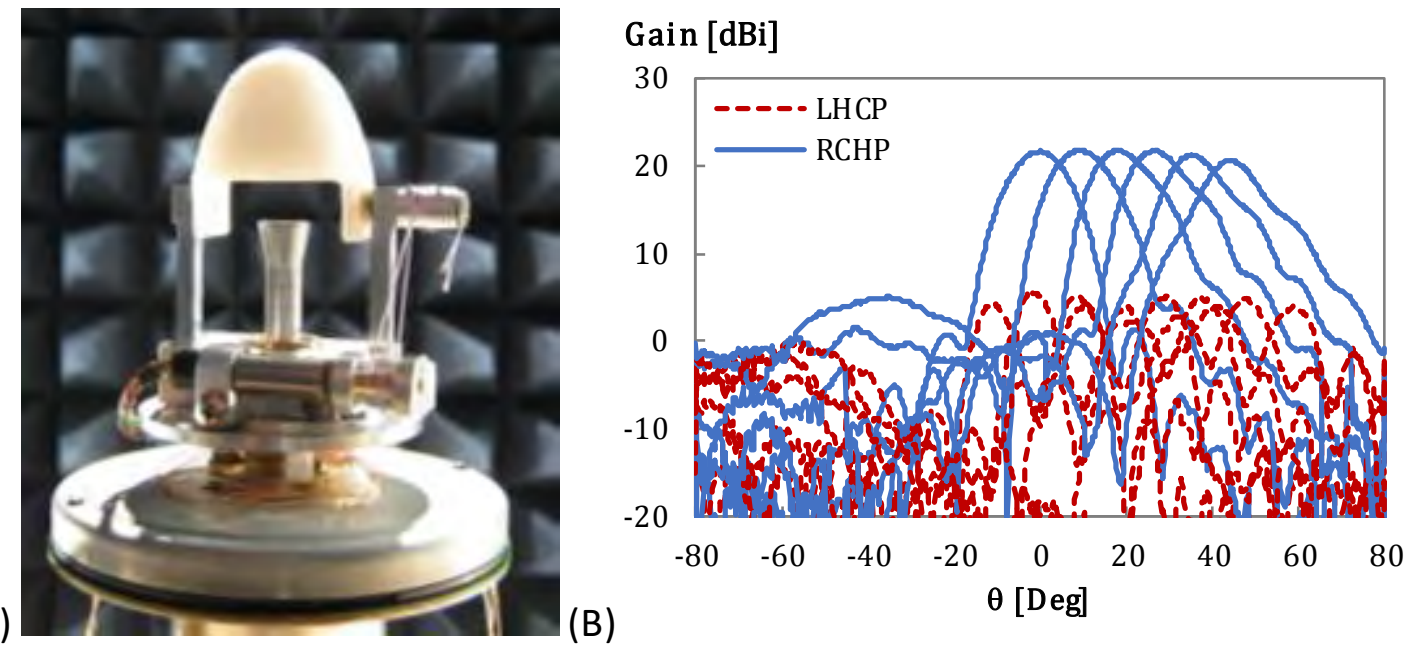

Figure $46-(A)$ photograph of the manufactured polyethylene lens plus horn feed; (B) measured radiation patterns of the lens antenna for several lens tilt angles.

\section{CONCLUSIONS}

Rapid prototyping techniques and the trend to move into millimetre and sub-millimeter waves are bringing back the interest on dielectric lens antennas, namely on integrated lens antenna configurations, taking advantage of the fast parallel advancements on lowcost fabrication of millimetre wave integrated circuits. This integration prompts for mass-market applications at these bands.

It was shown in this chapter that dielectric lenses are very flexible to accommodate demanding specifications and lens design is reasonably simple. Although a fast progress is being made on low-profile planar antennas employing phase shifting cells and metamaterials, dielectric lenses will remain a strong competitor at very small wavelengths owing to its effectiveness and simplicity for design and fabrication. 


\section{REFERENCES}

(Afasar 1990) Afsar MN, Li X, Chi H (1990) An automated $60 \mathrm{GHz}$ open resonator system for precision dielectric measurements. Microwave Theory and Techniques, IEEE Transactions on 38:1845-1853

(Artemenko 2013a) Artemenko A, Mozharovskiy A, Maltsev A et al. (2013) Experimental Characterization of E-Band Two-Dimensional Electronically BeamSteerable Integrated Lens Antennas. Antennas and Wireless Propagation Letters, IEEE 12:1188-1191

(Artemenko 2013b) Artemenko A, Maltsev A, Mozharovskiy A et al. (2013) MillimeterWave Electronically Steerable Integrated Lens Antennas for WLAN/WPAN Applications. Antennas and Propagation, IEEE Transactions on 61:1665-1671

(Bares 2007) Bares B, Sauleau R (2007) Design and optimisation of axisymmetric millimetre-wave shaped lens antennas with directive, secantsquared and conical beams. Microwaves, Antennas \& Propagation, IET 1:433-439

(Bisognin 2014) Bisognin A, Titz D, Ferrero F et al. (2014) 3D printed plastic $60 \mathrm{GHz}$ lens: Enabling innovative millimeter wave antenna solution and system. In: Microwave Symposium (IMS), 2014 IEEE MTT-S International. p 1-4

(Bor 2014) Bor J, Lafond O, Merlet $\mathrm{H}$ et al. (2014) Technological Process to Control the Foam Dielectric Constant Application to Microwave Components and Antennas. Components, Packaging and Manufacturing Technology, IEEE Transactions on 4:938-942

(Born 1959) Born M, Wolf E. (1959) Principles of Optics, Pergamon, New York

(Boriskin 2011) Boriskin AV, Vorobyov A, Sauleau R (2011) Two-Shell Radially Symmetric Dielectric Lenses as Low-Cost Analogs of the Luneburg Lens. Antennas and Propagation, IEEE Transactions on 59:3089-3093

(Chen 2005) Chen LF, Ong CK, Neo CP et al. (2005) Microwave Theory and Techniques for Materials Characterization. In: Microwave Electronics. John Wiley \& Sons, Ltd

(Cornbleet 1994) Cornbleet S (1994) Microwave and Geometrical Optics, Academic Press, London

(Costa 2007a) Costa JR, Fernandes CA (2007) Broadband slot feed for integrated lens antennas. IEEE Antennas Wirel. Propag. Lett. 6:396-400

(Costa 2007b) Costa JR, Fernandes CA (2007) Integrated Imaging Lens Antenna with Broadband Feeds. In: Antennas and Propagation, 2007. EuCAP 2007. The Second European Conference on. p 1-6

(Costa 2008a) Costa JR, Silveirinha MG, Fernandes CA (2008) Evaluation of a Double-Shell Integrated Scanning Lens Antenna. IEEE Antennas Wirel. Propag. Lett. 7:781-784 
(Costa 2008b) Costa JR, Fernandes CA, Godi G et al. (2008) Compact Ka-band lens antennas for LEO satellites. IEEE Trans. Antennas Propag. 56:12511258

(Costa 2009) Costa JR, Lima EB, Fernandes CA (2009) Compact Beam-Steerable Lens Antenna for $60-\mathrm{GHz}$ Wireless Communications. IEEE Trans. Antennas Propag. 57:2926-2933

(Costa 2010) Costa JR, Lima EB, Fernandes CA (2010) Antenna Phase Center Determination from Amplitude Measurements using a Focusing Lens. In: Antennas and Propagation Society International Symposium, 2010 IEEE.

(Do-Hoon 2010) Do-Hoon K, Werner DH (2010) Transformation Electromagnetics: An Overview of the Theory and Applications. Antennas and Propagation Magazine, IEEE 52:24-46

(Edwards 2012) Edwards JM, O'brient R, Lee AT et al. (2012) Dual-Polarized Sinuous Antennas on Extended Hemispherical Silicon Lenses. Antennas and Propagation, IEEE Transactions on 60:4082-4091

(Fernandes 1995) L. Fernandes, Developing a System Concept and Technologies for Mobile Broadband Communications, IEEE Personal Communications Magazine, 2, 54, 1995.

(Fernandes 1999a) Fernandes, C. A.; V. Brankovic; S. Zimmermman; Filipe, M.; L. Anunciada; "Dielectric Lens Antenna for Wireless Broadband Communications", Wireless Personal Communications, Vol. 10, No. 1, pp. 19 - 32, June, 1999.

(Fernandes 1999b) Fernandes CA (1999) Shaped dielectric lenses for wireless millimeterwave communications. Antennas and Propagation Magazine, IEEE 41:141-150

(Fernandes 2001) Fernandes CA, Anunciada LM (2001) Constant flux illumination of square cells for millimeter-wave wireless communications. Microwave Theory and Techniques, IEEE Transactions on 49:21372141

(Fernandes 2002) Fernandes CA (2002) Shaped-Beam Antennas. In Godara L (ed) Handbook of Antennas in Wireless Communications, CRC Press, New York, ch 15.

(Fernandes 2007) Fernandes CA, Costa JR, Van Der Vorst M (2007) Design of a shaped double-shell lens feed for a quasi-optical reflector system. In: 2007 IEEE Antennas and Propagation Society International Symposium, Vols 1-12. leee, New York, p 4858-4861

(Fernandes 2009) Fernandes CA, Costa JR (2009) Permittivity Measurement and Anisotropy Evaluation of Dielectric Materials at Millimeter-Waves. In: XIX Imeko World Congress: Fundamental and Applied Metrology, Proceedings. Imeko, Budapest, p 673-677 
(Fernandes 2010) Fernandes CA, Lima EB, Costa JR (2010) Broadband Integrated Lens for Illuminating Reflector Antenna With Constant Aperture Efficiency. Antennas and Propagation, IEEE Transactions on 58:38053813

(Fernandes 2011) Fernandes CA, Lima EB, Costa JR (2011) Tapered waveguide feed for integrated dielectric lens antenna performance tests. In: EUROCON International Conference on Computer as a Tool (EUROCON), 2011 IEEE. p 1-4

(Filipovic 1993) Filipovic DF, Gearhart SS, Rebeiz GM (1993) Double-slot antennas on extended hemispherical and elliptical silicon dielectric lenses. Microwave Theory and Techniques, IEEE Transactions on 41:17381749

(Filipovic 1997) Filipovic DF, Gauthier GP, Raman S et al. (1997) Off-axis properties of silicon and quartz dielectric lens antennas. Antennas and Propagation, IEEE Transactions on 45:760-766

(Fuchs 2006) Fuchs B, Lafond O, Rondineau S et al. (2006) Design and characterization of half Maxwell fish-eye lens antennas in millimeter waves. Microwave Theory and Techniques, IEEE Transactions on 54:2292-2300

(Fuchs 2007a) Fuchs B, Le Coq L, Lafond O et al. (2007) Design Optimization of Multishell Luneburg Lenses. Antennas and Propagation, IEEE Transactions on 55:283-289

(Fuchs 2007b) Fuchs B, Lafond O, Rondineau S et al. (2007) Off-Axis Performances of Half Maxwell Fish-Eye Lens Antennas at $77 \mathrm{GHz}$. Antennas and Propagation, IEEE Transactions on 55:479-482

(Fuchs 2008a) Fuchs B, Lafond O, Palud S et al. (2008) Comparative Design and Analysis of Luneburg and Half Maxwell Fish-Eye Lens Antennas. Antennas and Propagation, IEEE Transactions on 56:3058-3062

(Fuchs 2008b) Fuchs B, Palud S, Le Coq L et al. (2008) Scattering of Spherically and Hemispherically Stratified Lenses Fed by Any Real Source. Antennas and Propagation, IEEE Transactions on 56:450-460

(Ka Fai 2014) Ka Fai C, Rui L, Cheng J et al. (2014) 77-GHz Automotive Radar Sensor System With Antenna Integrated Package. Components, Packaging and Manufacturing Technology, IEEE Transactions on 4:352-359

(Kay 1965) Kay K (1965) Electromagnetic Theory and Geometrical Optics, Interscience Publishers, New York

(Kelleher 1961) Kelleher K (1961) Scanning Antennas, Chap 15 in Antenna Engineering Handbook, H.Jasik (Ed.), McGraw-Hill, New York.

(Kim 1998) Kim KW, Rahmat-Samii Y (1998) Spherical Luneburg lens antennas: engineering characterizations including air gap effects. In: Antennas and Propagation Society International Symposium, 1998. IEEE. $p$ 2062-2065 vol.2064 
(Kolundzija 2002) Kolundzija B., Djordjevic A. (2002) Electromagnetic Modelling of Composite Metallic and Dielectric Structures, Artech House, Norwood

(Komiyama 1991) Komiyama B, Kiyokawa M, Matsui T (1991) Open resonator for precision dielectric measurements in the $100 \mathrm{GHz}$ band. Microwave Theory and Techniques, IEEE Transactions on 39:1792-1796

(Komljenovic 2010) Komljenovic T, Sauleau R, Sipus Z et al. (2010) Layered CircularCylindrical Dielectric Lens Antennas\&\#x2014;Synthesis and Height Reduction Technique. Antennas and Propagation, IEEE Transactions on $58: 1783-1788$

(Hailu 2009) Hailu DM, Ehtezazi IA, Safavi-Naeini S (2009) Fast Analysis of Terahertz Integrated Lens Antennas Employing the Spectral Domain Ray Tracing Method. Antennas and Wireless Propagation Letters, IEEE 8:37-39

(Hailu 2011) Hailu DM, Ehtezazi IA, Neshat M et al. (2011) Hybrid Spectral-Domain Ray Tracing Method for Fast Analysis of Millimeter-Wave and Terahertz-Integrated Antennas. Terahertz Science and Technology, IEEE Transactions on 1:425-434

(Hirvonen 1996) Hirvonen TM, Vainikainen P, Lozowski A et al. (1996) Measurement of dielectrics at $100 \mathrm{GHz}$ with an open resonator connected to a network analyzer. Instrumentation and Measurement, IEEE Transactions on 45:780-786

(Lima 2008) Lima E, Costa JR, Silveirinha MG et al. (2008) ILASH - Software tool for the design of integrated lens antennas. In: Antennas and Propagation Society International Symposium, 2008. AP-S 2008. IEEE. p 1-4

(Ling 1986) Ling H, Chou R, Lee S (1986) Shooting and bouncing rays: Calculating RCS of an arbitrary cavity. In: Antennas and Propagation Society International Symposium, 1986. p 293-296

(Llombart 2013) Llombart N, Lee C, Alonso-Delpino M et al. (2013) Silicon Micromachined Lens Antenna for THz Integrated Heterodyne Arrays. Terahertz Science and Technology, IEEE Transactions on 3:515-523

(Lodge 1888) Lodge OJ, Howard JL (1888) On Electric Radiation and its Concentration by Lenses. Proceedings of the Physical Society of London 10:143

(Luneburg 1943) Luneburg R. K. (1943), US Patent 2,328,157

(Maciel 1989) Maciel JJ, Felsen LB (1989) Systematic study of fields due to extended apertures by Gaussian beam discretization. Antennas and Propagation, IEEE Transactions on 37:884-892

(Mateo-Segura 2014) Mateo-Segura C, Dyke A, Dyke H et al. (2014) Flat Luneburg Lens via Transformation Optics for Directive Antenna Applications. Antennas and Propagation, IEEE Transactions on 62:1945-1953

(Maxwell 1860) Maxwell J C (1860) Scientific Papers, I, New York, Dover Publications 
(Min 2014) Min L, Wei-Ren N, Kihun C et al. (2014) A 3-D Luneburg Lens Antenna Fabricated by Polymer Jetting Rapid Prototyping. Antennas and Propagation, IEEE Transactions on 62:1799-1807

(Mosallaei 2001) Mosallaei H, Rahmat-Samii Y (2001) Nonuniform Luneburg and twoshell lens antennas: radiation characteristics and design optimization. Antennas and Propagation, IEEE Transactions on 49:6069

(Naruse 2013) Naruse M, Sekimoto Y, Noguchi T et al. (2013) Optical Efficiencies of Lens-Antenna Coupled Kinetic Inductance Detectors at $220 \mathrm{GHz}$. Terahertz Science and Technology, IEEE Transactions on 3:180-186

(Neto 1998) Neto A, Maci S, De Maagt PJI (1998) Reflections inside an elliptical dielectric lens antenna. Microwaves, Antennas and Propagation, IEE Proceedings - 145:243-247

(Neto 1999) Neto A, Borselli L, Maci S et al. (1999) Input impedance of integrated elliptical lens antennas. Microwaves, Antennas and Propagation, IEE Proceedings 146:181-186

(Neto 2010a) Neto A (2010) UWB, Non Dispersive Radiation From the Planarly Fed Leaky Lens Antenna - Part 1: Theory and Design. Antennas and Propagation, IEEE Transactions on 58:2238-2247

(Neto 2010b) Neto A, Monni S, Nennie F (2010) UWB, Non Dispersive Radiation From the Planarly Fed Leaky Lens Antenna - Part II: Demonstrators and Measurements. Antennas and Propagation, IEEE Transactions on 58:2248-2258

(Nguyen 2010) Nguyen NT, Sauleau R, Martinez Perez CJ et al. (2010) Finitedifference time-domain simulations of the effects of air gaps in double-shell extended hemispherical lenses. Microwaves, Antennas \& Propagation, IET 4:35-42

(Nguyen 2011) Nguyen NT, Sauleau R, Ettorre M et al. (2011) Focal Array Fed Dielectric Lenses: An Attractive Solution for Beam Reconfiguration at Millimeter Waves. Antennas and Propagation, IEEE Transactions on 59:2152-2159

(Ngoc Tinh 2009) Ngoc Tinh N, Sauleau R, Perez CJM (2009) Very Broadband Extended Hemispherical Lenses: Role of Matching Layers for Bandwidth Enlargement. Antennas and Propagation, IEEE Transactions on 57:1907-1913

(Ngoc Tinh 2010) Ngoc Tinh N, Delhote N, Ettorre M et al. (2010) Design and Characterization of $60-\mathrm{GHz}$ Integrated Lens Antennas Fabricated Through Ceramic Stereolithography. Antennas and Propagation, IEEE Transactions on 58:2757-2762

(Ngoc Tinh 2011) Ngoc Tinh N, Sauleau R, Le Coq L (2011) Reduced-Size Double-Shell Lens Antenna With Flat-Top Radiation Pattern for Indoor Communications at Millimeter Waves. Antennas and Propagation, IEEE Transactions on 59:2424-2429 
(Ngoc Tinh 2013) Ngoc Tinh N, Boriskin AV, Rolland A et al. (2013) Shaped Lens-Like Dome for UWB Antennas With a Gaussian-Like Radiation Pattern. Antennas and Propagation, IEEE Transactions on 61:1658-1664

(Nikolic 2012) Nikolic N, James GL, Hellicar A et al. (2012) Quarter-sphere Luneburg lens scanning antenna. In: Antenna Technology and Applied Electromagnetics (ANTEM), 2012 15th International Symposium on. p 1-4

(Olver 1988) Olver A, Clarricoats P, Kishk A, Shafai L (1998) Microwave Horns and Feeds, Chap. 11, IEEE Press, New York

(Pasqualini 2004) Pasqualini D, Maci S (2004) High-frequency analysis of integrated dielectric lens antennas. Antennas and Propagation, IEEE Transactions on 52:840-847

(Pavacic 2006) Pavacic AP, Del Rio DL, Mosig JR et al. (2006) Three-dimensional raytracing to model internal reflections in off-axis lens antennas. Antennas and Propagation, IEEE Transactions on 54:604-612

(Peterson 1998) Peterson AF, Ray SL, Mittra R (1998) Computational Methods of Electromagnetics, IEEE Press, New York

(Petosa 2000) Petosa A, Ittipiboon A (2000) Shadow blockage effects on the aperture efficiency of dielectric Fresnel lenses. Microwaves, Antennas and Propagation, IEE Proceedings 147:451-454

(Piksa 2011) Piksa P, Zvanovec S, Cerny P (2011) Elliptic and Hyperbolic Dielectric Lens Antennas in mm-Waves. RADIOENGINEERING 20:271

(Rebeiz 1992) Rebeiz GM (1992) Millimeter-wave and terahertz integrated circuit antennas. Proceedings of the IEEE 80:1748-1770

(Rolland 2011) Rolland A, Sauleau R, Le Coq L (2011) Flat-Shaped Dielectric Lens Antenna for $60-\mathrm{GHz}$ Applications. Antennas and Propagation, IEEE Transactions on 59:4041-4048)

(Rutledge 1983) Rutledge D, Neikirk D, Kasilingam D (1983) Integrated circuit antennas. Infrared and Millimeter-Waves, K. Button, Ed. New York: Academic, vol. 10, pp. 1-90

(Salema 1998) Salema C, Fernandes C, Jha R (1998) Solid Dielectric Horns, Chap. 7, Artech House, Boston

(Sanford 1994) Sanford JR (1994) Scattering by spherically stratified microwave lens antennas. Antennas and Propagation, IEEE Transactions on 42:690698

(Sato 2002) Sato K, Ujiie H (2002) A plate Luneberg lens with the permittivity distribution controlled by hole density. Electronics and Communications in Japan (Part I: Communications) 85:1-12

(Sauleau 2006) Sauleau R, Bares B (2006) A complete procedure for the design and optimization of arbitrarily shaped integrated lens antennas. Antennas and Propagation, IEEE Transactions on 54:1122-1133 
(Semenov 2007) Semenov AD, Richter H, Hubers HW et al. (2007) Terahertz Performance of Integrated Lens Antennas With a Hot-Electron Bolometer. Microwave Theory and Techniques, IEEE Transactions on 55:239-247

(Silveirinha 2000) Silveirinha MGMV, Fernandes CA (2000) Shaped double-shell dielectric lenses for wireless millimeter wave communications. In: Antennas and Propagation Society International Symposium, 2000. IEEE. p 1674-1677 vol.1673

(Silveirinha 2014) Silveirinha MG, Fernandes CA, Costa JR (2014) A Graphical Aid for the Complex Permittivity Measurement at Microwave and Millimeter Wavelengths. Microwave and Wireless Components Letters, IEEE 24:421-423

(Silver 1984) Silver S. (1984) Microwave Antenna Theory and Design. Peter Pereginus, London

(Trichopoulos 2010) Trichopoulos GC, Mumcu G, Sertel K et al. (2010) A Novel Approach for Improving Off-Axis Pixel Performance of Terahertz Focal Plane Arrays. Microwave Theory and Techniques, IEEE Transactions on 58:2014-2021

(Trichopoulos 2013) Trichopoulos GC, Mosbacker HL, Burdette D et al. (2013) A Broadband Focal Plane Array Camera for Real-time THz Imaging Applications. Antennas and Propagation, IEEE Transactions on 61:1733-1740

(Van Der Vorst 1999) Van Der Vorst MJM, De Maagt PJL, Herben MHaJ (1999) Effect of internal reflections on the radiation properties and input admittance of integrated lens antennas. Microwave Theory and Techniques, IEEE Transactions on 47:1696-1704

(Van Der Vorst 2001) Van Der Vorst MJM, De Maagt PJI, Neto A et al. (2001) Effect of internal reflections on the radiation properties and input impedance of integrated lens antennas-comparison between theory and measurements. Microwave Theory and Techniques, IEEE Transactions on 49:1118-1125

(Van Der Vorst 2002) Van Der Vorst MJM, De Maagt PJL (2002) Efficient body of revolution finite-difference time-domain modeling of integrated lens antennas. Microwave and Wireless Components Letters, IEEE $12: 258-260$

(Wu 2001) Wu X, Eleftheriades G, Van Deventer-Perkins T, (2001) Design and Characterization of Single- and Multiple-Beam MM-Wave Circularly Polarized Substrate Lens Antennas for Wireless Communications, Microwave Theory and Techniques, IEEE Transactions on 49:431-441

(Xue 2007) Xue L, Fusco VF (2007) 24 GHz automotive radar planar Luneburg lens. Microwaves, Antennas \& Propagation, IET 1:624-628

(Yurduseven 2014)Yurduseven O, Cavallo D, Neto A (2014) Wideband Dielectric Lens Antenna With Stable Radiation Patterns Fed by Coherent Array of 
Connected Leaky Slots. Antennas and Propagation, IEEE Transactions on 62:1895-1902 